\title{
UMA SOLUÇÃO PARA O PROBLEMA DE SCHROEDER-BERNSTEIN PARA ESPAÇOS DE BANACH
}

\author{
Roberto Nicolosi \\ DISSERTAÇÃO APRESENTADA \\ $\mathrm{AO}$ \\ INSTITUTO DE MATEMÁTICA E ESTATÍSTICA \\ DA \\ UNIVERSIDADE DE SÃO PAULO \\ PARA \\ OBTENÇÃO DO GRAU DE MESTRE \\ EM \\ MATEMÁTICA \\ Área de Concentração: Análise Matemática \\ Orientador: Prof. Dr. Elói Medina Galego \\ - São Paulo, março de 2002 -
}




\section{UMA SOLUÇÃO PARA O PROBLEMA \\ DF, SCHROFDER-BERNSTEIN PARA \\ ESPAÇOS DE BANACH}

Este exemplar corresponde à redação final da dissertação apresentada por Roberto Nicolosi, devidamente corrigida e aprovada pela Comissão Julgadora

São Paulo, 09 maio de 2002.

\section{Comissão Julgadora}

- Prof. Dr. Elói Medina Galego (Orientador) - IME-USP

- Prof. Dr. Raimundo Luiz de Alencar - ITA

- Prof. Dr. Jorge Túlio Mujica Ascui - IMECC-UNICAMP 


\section{Agradecimentos}

A meu orientador, Professor Doutor Elói Medina Galego, pela orientação e assistência na elaboração deste trabalho. Meu profundo agradecimento.

À minha esposa, Teresinha Cristina, pelo incentivo e incondicional apoio.

Ao Sr. Walter Vicente Fernandes, pela dedicação e pelo excelente trabalho de digitação.

A todos os professores, colegas de curso e amigos que direta ou indiretamente contribuíram para a realização deste trabalho. 


\section{Resumo}

O objetivo deste trabalho é apresentar a primeira solução para o problema de Schroeder-Bernstein para espaços de Banach construída por W. T. Gowers.

Dois espaços não isomorfos são construídos, tais que cada um é um subespaço complementado do outro. Além disso, os espaços são da forma $Z$ e $Z^{2}$, onde $Z$ é isomorfo a $Z^{3}$.

\section{Abstract}

The purpose of this work is to present the first solution to the SchroederBernstein problem for Banach spaces constructed by Gowers, W. T.

Two non-isomorphic Banach spaces are constructed, such that either is a complemented subspace of the other. Moreover, the spaces are of the form $Z$ and $Z^{2}$, where $Z$ is isomorphic to $Z^{3}$. 
A meu filho Victor 


\section{Sumário}

Notações

Introdução

0 Preliminares

6

1 O espaço W

2 Uma solução para o problema de Schroeder-Bernstein para espaços de Banach

Problemas em aberto

Referências bibliográficas 


\section{Notações}

Seja $X$ um espaço de Banach sobre $\mathbb{R}$; se $Y$ é um subespaço fechado de $X$, escrevemos $Y<X$.

Sejam $X$ e $Y$ espaços de Banach, dizemos que $X$ é isomorfo a $Y$ e escrevemos $X \sim Y$, se existe uma aplicação linear limitada, bijetora, $T: X \rightarrow Y$, com inversa limitada.

Sejam $X$ e $Y$ espaços de Banach, dizemos que $X$ contém uma cópia isomorfa de $Y$ e escrevemos $Y \hookrightarrow X$, se existe $Z, Z<X \operatorname{com} Z \sim Y$.

Sejam $X$ e $Y$ espaços de Banach, dizemos que $X$ contém uma cópia isomorfa de $Y$ complementada em $X$ e escrevemos $Y \stackrel{c}{\hookrightarrow} X$, se existem $Z<X, Z \sim Y$ e $W<X$, tais que $X \sim W \oplus Z$, isto é, $X$ é a soma direta de $W$ e $Z$.

Sejam $X$ e $Y$ espaços de Banach, escrevemos $X \oplus_{\infty} Y$ para designar a soma direta externa de $X$ e $Y$, isto é,

$$
X \underset{\infty}{\oplus Y}=\{(x, y): x \in X \text { e } y \in Y\}, \quad \operatorname{com}\|(x, y)\|=\operatorname{máx}\{\|x\|,\|y\|\} .
$$

Seja $X$ um espaço de Banach, então $X^{n}$ denota a soma de $n$ cópias de $X, n \in \mathbb{N}^{*}$.

Seja $X$ um espaço de Banach, $B(X)$ indica o espaço de Banach dos operadores lineares limitados, $T: X \rightarrow X$, com a norma $\|T\|=\sup \{\|T(x)\|:\|x\| \leq 1\}$. 
Seja $X$ um espaço de Banach, indicamos por $X^{*}$ o espaço de Banach de todos os funcionais lineares limitados de $X$ (dual topológico de $X$ ). Escrevemos $x^{*}$, para indicar um funcional linear limitado de $X$.

Seja $X$ um espaço de Banach, por $\operatorname{dim} X$ denotamos a dimensão linear do espaço $X$, isto é, $\operatorname{dim} X$ pode ser finita ou infinita.

Sejam $X$ um espaço normado e $V \subset X$, escrevemos $[V]$ para representar o fecho do subespaço linear gerado pelo conjunto $V$.

Sejam $X$ um espaço de Banach e $T \in B(X)$, por $\operatorname{ker} T$ denotamos o núcleo de $T$ e por $\operatorname{Im} T$ denotamos a imagem de $T$.

Denotamos por $c_{00}$, o espaço vetorial das seqüências de escalares com apenas um número finito de coordenadas não nulas, e a base vetorial unitária desse espaço por $\left(e_{n}\right)_{n=1}^{\infty}$.

Um intervalo $E$ de inteiros é um subconjunto de $\mathbb{N}$ da forma $\{a, a+1, \ldots, b\}$ para algum $a, b \in \mathbb{N}$.

Sejam $E$ e $F$ intervalos, escrevemos $E<F$, se máx $E<\min F$.

Sejam $x=\sum_{i=1}^{\infty} a_{i} e_{i} \in c_{00}$ e $E$ um intervalo de $\mathbb{N}$. Denotamos por $E(x)$ o vetor $\sum_{i \in E} a_{i} e_{i}$

Por $\operatorname{supp}(x)$ denotamos o suporte de um vetor $x=\sum_{i=1}^{\infty} a_{i} e_{i} \in c_{00}$, isto é, $\operatorname{supp}(x)=\left\{i \in \mathbb{N}: a_{i} \neq 0\right\}$ e por ran $(x)$ denotamos o menor intervalo $E$, contendo o seu suporte.

Para $x, y \in c_{00}$, escrevemos $x<y$ quando máxsupp $(x)<\operatorname{minsupp}(y)$ ou equivalente, $\operatorname{ran}(x)<\operatorname{ran}(y)$. Se $x_{1}<x_{2}<\cdots<x_{n}$, dizemos que $x_{1}, x_{2}, \ldots, x_{n}$ 
são sucessivos.

Denotamos por $c_{0}$, o espaço de Banach das seqüências de escalares, cujo limite é igual a zero, ou seja,

$$
c_{0}=\left\{\left(a_{1}, a_{2}, \ldots, a_{n}, \ldots\right): a_{n} \in \mathbb{R} \text { e } \lim _{n \rightarrow+\infty} a_{n}=0\right\}
$$

com a norma $\left\|\left(a_{n}\right)_{n=1}^{\infty}\right\|_{\infty}=\operatorname{máx}\left\{\left|a_{n}\right|: n \in \mathbb{N}\right\}$.

Por $\ell_{p}$ denotamos o espaço de Banach das seqüências $p$-somáveis, $1 \leq p<\infty$, isto é,

$$
\ell_{p}=\left\{\left(a_{n}\right)_{n=1}^{\infty}: a_{n} \in \mathbb{R} \text { e } \sum_{n=1}^{\infty}\left|a_{n}\right|^{p}<\infty\right\}
$$

com a norma $\left\|\left(a_{n}\right)_{n=1}^{\infty}\right\|_{p}=\left(\sum_{n=1}^{\infty}\left|a_{n}\right|^{p}\right)^{\frac{1}{p}}$

Por $\ell_{\infty}$ denotamos o espaço de Banach das seqüências limitadas, isto é,

$$
\ell_{\infty}=\left\{\left(a_{n}\right)_{n=1}^{\infty}: a_{n} \in \mathbb{R} \text { e } \sup _{n \in \mathbb{N}}\left|a_{n}\right|<\infty\right\}
$$

com a norma $\left\|\left(a_{n}\right)_{n=1}^{\infty}\right\|=\sup \left\{\left|a_{n}\right|: n \in \mathbb{N}\right\}$.

Se $\left(X_{n}\right)_{n=1}^{\infty}$ for uma seqüência de espaços de Banach, $\left(\sum_{n=1}^{\infty} X_{n}\right)_{0}$ é o espaço de Banach das seqüências $x=\left(x_{1}, x_{2}, \ldots, x_{n}, \ldots\right), x_{n} \in X_{n}$, tais que $\lim _{n \rightarrow+\infty}\left\|x_{n}\right\|=0$ com a norma $\|x\|=\operatorname{máx}\left\{\left\|x_{n}\right\|: n \in \mathbb{N}\right\}$ e $\left(\sum_{n=1}^{\infty} X_{n}\right)_{\ell_{p}}, 1 \leq p<\infty$, é o espaço de Banach das seqüências $x=\left(x_{1}, x_{2}, \ldots, x_{n}, \ldots\right), x_{n} \in X_{n}$, tais que $\sum_{n=1}^{\infty}\left\|x_{n}\right\|^{p}<\infty$, com a norma $\|x\|=\left(\sum_{n=1}^{\infty}\left\|x_{n}\right\|^{p}\right)^{\frac{1}{p}}$.

Usamos $a \vee b$ para indicar máx $\{a, b\}$, com $a, b \in \mathbb{R}$.

Para indicar o término de uma demonstração, usamos o símbolo 


\section{Introdução}

O principal objetivo desta dissertação é apresentar detalhadamente a primeira solução para o problema de Schroeder-Bernstein para espaços de Banach, que consiste na seguinte questão: "Sejam $X$ e $Y$ espaços de Banach. Se $X \stackrel{c}{\hookrightarrow} Y$ e $Y \stackrel{c}{\hookrightarrow} X$, então $X \sim Y$ ?" A solução que apresentaremos (contra-exemplo) foi construída por W. T. Gowers em 1996.

O problema de Schroeder-Bernstein surge naturalmente, veja [Ca], a partir dos seguintes métodos de decomposição em espaços de Banach:

(M1) Sejam $X$ e $Y$ espaços de Banach. Se $X \stackrel{c}{\hookrightarrow} Y, Y \stackrel{c}{\hookrightarrow} X, X^{2} \sim X$ e $Y^{2} \sim Y$, então $X \sim Y$.

(M2) Sejam $X$ e $Y$ espaços de Banach. Se $X \stackrel{c}{\hookrightarrow} Y, Y \stackrel{c}{\hookrightarrow} X,\left(\sum_{n=1}^{\infty} X_{n}\right)_{0} \sim X$ ou $\left(\sum_{n=1}^{\infty} X_{n}\right)_{\ell_{p}} \sim X$, então $X \sim Y$.

Esses métodos foram apresentados por A. Pelczynski na década de 60 , e têm até hoje um papel muito importante na teoria isomorfa de espaços de Banach.

O contra-exemplo para o problema de Schroeder-Bernstein, proposto por W. T. Gowers, é formado por dois espaços de Banach da forma: $X \oplus \mathrm{W}$ e W. O espaço $X$ é a versão real do primeiro espaço de Banach hereditariamente indecomponível, 
construído por W. T. Gowers e B. Maurey em 1993, veja [G-M1] e [C].

O espaço $\mathbf{W}$ é da forma $\mathbf{W}=\sum_{i=1}^{\infty} \oplus X_{i}$, onde cada $X_{i}$ é uma cópia isométrica de $X$ e a soma direta infinita é uma decomposição de Schauder de W.

Escrevendo $Z=X \oplus \mathrm{W}$, mostraremos que $Z \stackrel{c}{\hookrightarrow} Z^{2}, Z^{2} \stackrel{c}{\hookrightarrow} Z$ e também $Z^{3} \sim Z$ (Lema 1.22), mas pelo Teorema 2.22, obteremos $Z \nsim Z^{2}$, $\log$ o $Z$ e $Z^{2}$ formarão o contra-exemplo para o problema de Schroeder-Bernstein. Além disso, obteremos o primeiro espaço de Banach $Z, \operatorname{com} Z \not Z^{2}$ mas $Z \sim Z^{3}$.

Finalmente, observamos que outras soluções para o problema de SchroederBernstein foram apresentadas por W. T. Gowers e B. Maurey em [G-M2]. 


\section{Capítulo 0}

\section{Preliminares}

Neste capítulo apresentaremos definições e resultados sobre a teoria dos espaços de Banach e também sobre o espaço $X$, construído por W. T. Gowers e B. Maurey em [G-M1]. Estes resultados e definições serão utilizados nos capítulos seguintes.

Definição 0.1 Uma seqüência $\left(x_{n}\right)_{n=1}^{\infty}$ em um espaço de Banach $E$ é uma base de Schauder de $E$ se, e somente se, para cada $x \in E$, existe uma única seqüência de escalares $\left(a_{n}\right)_{n=1}^{\infty}$ tal que $x=\sum_{n=1}^{\infty} a_{n} x_{n}$.

Pela proposição 1.a.2 [L-T, pág. 1], as projeções $P_{n}: E \rightarrow E$ definidas por $P_{n}\left(\sum_{i=1}^{\infty} a_{i} x_{i}\right)=\sum_{i=1}^{n} a_{i} x_{i}$ são limitadas e $\sup \left\{\left\|P_{n}\right\|: n \in \mathbb{N}\right\}<\infty$. O número $\sup \left\{\left\|P_{n}\right\|: n \in \mathbb{N}\right\}$ é chamado de constante básica de $\left(x_{n}\right)_{n=1}^{\infty}$ e quando esse número for igual a 1, a base é chamada monótona.

Definição 0.2 Uma seqüência $\left(x_{n}\right)_{n=1}^{\infty}$ que é base de Schauder do subespaço fechado gerado por ela, isto é, $\left[\left(x_{n}\right)_{n=1}^{\infty}\right]$, é chamada de seqüência básica.

Teorema 0.3 (Ga, pág. 47) Seja $\left(x_{n}\right)_{n=1}^{\infty}$ uma seqüência de elementos não nulos no espaço de Banach E. Então $\left(x_{n}\right)_{n=1}^{\infty}$ é uma seqüência básica se, e somente se, existe um número real positivo $k$, tal que para qualquer seqüência de escalares $\left(a_{n}\right)_{n=1}^{\infty}$ e inteiros $m<n$, tem-se $\left\|\sum_{i=1}^{m} a_{i} x_{i}\right\| \leq k\left\|\sum_{i=1}^{n} a_{i} x_{i}\right\|$. 
Definição 0.4 Sejam $E$ um espaço de Banach, $\left(x_{n}\right)_{n=1}^{\infty}$ uma seqüência básica em $E,\left(p_{n}\right)_{n=1}^{\infty}$ uma seqüência estritamente crescente de inteiros positivos e $\left(a_{n}\right)_{n=1}^{\infty}$ uma. seqüência de números reais. Uma seqüência $\left(u_{j}\right)_{j=1}^{\infty}$ dada por $u_{j}=\sum_{n=p_{j}+1}^{p_{j+1}} a_{n} x_{n}$, com $u_{j} \neq 0, \forall j \in \mathbb{N}$, é chamada de base de bloco de $\left(x_{n}\right)_{n=1}^{\infty}$.

Observação 0.5 Se $\left(x_{n}\right)_{n=1}^{\infty}$ é uma seqüência básica de $E$, então pelo Teorema 0.3 toda base de bloco $\left(u_{j}\right)_{j=1}^{\infty}$ de $\left(x_{n}\right)_{n=1}^{\infty}$ é também uma seqüência básica de $E$. O subespaço fechado de $E$, gerado por $\left(u_{j}\right)_{j=1}^{\infty},\left[\left(u_{j}\right)_{j=1}^{\infty}\right]$, é chamado de subespaço de bloco de $E$.

Definição 0.6 Sejam $E$ um espaço de Banach e $\left(x_{n}\right)_{n=1}^{\infty}$ uma seqüência básica em $E$. Para todo inteiro positivo $j$, o funcional linear limitado $f_{j}^{*}$ definido por $f_{j}^{*}\left(\sum_{i=1}^{\infty} a_{i} x_{i}\right)=a_{j}$ é chamado de funcional biortogonal ou funcional coeficiente da seqüência básica $\left(x_{n}\right)_{n=1}^{\infty}$.

Definição 0.7 Duas bases de Schauder $\left(x_{n}\right)_{n=1}^{\infty}$ e $\left(y_{n}\right)_{n=1}^{\infty}$ de espaços de Banach $E$ e $F$ respectivamente, são ditas equivalentes se $\sum_{n=1}^{\infty} a_{n} x_{n}$ converge se, e somente se, $\sum_{n=1}^{\infty} b_{n} x_{n}$ também converge. Escrevemos $\left(x_{n}\right)_{n=1}^{\infty} \sim\left(y_{n}\right)_{n=1}^{\infty}$.

Proposição 0.8 (Ga, pág. 50) Sejam E e F espaços de Banach. Uma base de Schauder $\left(x_{n}\right)_{n=1}^{\infty}$ de E é equivalente a uma base de Schauder $\left(y_{n}\right)_{n=1}^{\infty}$ de F se, e somente se, existe um isomorfismo $T: E \rightarrow F$, tal que $T\left(x_{n}\right)=y_{n}, \forall n \in \mathbb{N}$.

Proposição 0.9 (L-T, pág. 53) Seja E um dos espaços de Banach, $c_{0}$ ou $\ell_{p}, 1 \leq$ $p<\infty, e\left(z_{n}\right)_{n=1}^{\infty}$ uma base de bloco de $\left(e_{n}\right)_{n=1}^{\infty}$ normalizada em E. Então $\left(z_{n}\right)_{n=1}^{\infty}$ é equivalente a $\left(e_{n}\right)_{n=1}^{\infty} e\left[\left(z_{n}\right)_{n=1}^{\infty}\right]$ é isométrico a $\mathrm{E}$.

Proposição 0.10 (L-T, pág. 7) Seja $\left(x_{n}\right)_{n=1}^{\infty}$ uma base de Schauder de um espaço de Banach E e $y_{k}=\sum_{n=1}^{\infty} a_{n}^{k} x_{n}, k=1,2, \ldots$, uma seqüência de vetores tais que:

$$
\lim _{k \rightarrow+\infty} \sup \left\|y_{k}\right\|>0 \quad e \quad \lim _{k \rightarrow+\infty} a_{n}^{k}=0 \text {. }
$$


Então, existe uma subseqüência $\left(y_{k_{j}}\right)_{j=1}^{\infty}$ de $\left(y_{k}\right)_{k=1}^{\infty}$, que é equivalente a uma base de bloco de $\left(x_{n}\right)_{n=1}^{\infty}$.

Observação 0.11 (Ga, pág. 52) Seja F um subespaço de dimensão infinita de um espaço de Banach $E$ e $\left(x_{n}\right)_{n=1}^{\infty}$ uma base de Schauder de $E$, então para todo inteiro positivo $p$ existe $y \in F$ tal que $y=\sum_{n=p+1}^{\infty} a_{n} x_{n}$, com $\|y\|=1$.

Proposição 0.12 (Ho, pág. 189) S'eja F um espaço de Banach reflexivo, então todo subespaço vetorial fechado $F$ de $E$ é reflexivo.

Proposição 0.13 (Ho, pág. 183) Sejam E um espaço normado, F um subespaço vetorial fechado de $E, F^{0}$ o conjunto de todos os funcionais lineares $x^{*} \in E^{*}$, tais que $x^{*}(x)=0$ para todo vetor $x \in F$ e $x_{1} \in E$ um vetor com $x_{1} \notin F$. Então existe $x^{*} \in F^{0}$, tal que $\left\|x^{*}\right\|=1, x^{*}\left(x_{1}\right)=\delta=d\left(x_{1}, F\right)=\inf \left\{\left\|x_{1}-x\right\|: x \in F\right\}$.

Corolário 0.14 (Ho, pág. 182) Sejam E um espaço normado, E $E_{0}$ um subespaço linear de $F$ e $f_{0}: E_{0} \rightarrow \mathbb{R}$ um funcional linear limitado. Então existe um funcional linear limitado $f: E \rightarrow \mathbb{R}$, tal que $f(x)=f_{0}(x)$ para todo $x \in E_{0}$ com $\|f\|=\left\|f_{0}\right\|$.

Definição 0.15 Sejam $E$ um espaço de Banach e $\left(x_{n}\right)_{n=1}^{\infty}$ uma base de Schauder de $E$.

(a) $\left(x_{n}\right)_{n=1}^{\infty}$ é contrátil se para todo $x^{*} \in E^{*}$ sendo $\left.x^{*}\right|_{\left[\left(x_{i}\right)_{i=n}^{\infty}\right]}:\left[\left(x_{i}\right)_{i=n}^{\infty}\right] \rightarrow \mathbb{R}$ $\forall n \in \mathbb{N}$, a restrição de $x^{*}$ ao fecho do subespaço gerado por $\left(x_{i}\right)_{i=n}^{\infty}$, tem-se $\|\left. x^{*}\right|_{\left[\left(x_{i}\right)_{i=n}^{\infty}\right]} \stackrel{n \rightarrow+\infty}{\longrightarrow} 0$

(b) $\left(x_{n}\right)_{n=1}^{\infty}$ é limitadamente completa, se para qualquer seqüência de escalares $\left(a_{i}\right)_{i=1}^{\infty} \operatorname{com} \sup \left\{\left\|\sum_{i=1}^{n} a_{i} x_{i}\right\|: n \in \mathbb{N}\right\}<\infty$, a série $\sum_{i=1}^{\infty} a_{i} x_{i}$ é convergente.

Proposição 0.16 (L-T, pág. 8) Sejam $\left(x_{n}\right)_{n=1}^{\infty}$ uma base de Schauder de um espaço de Banach $E \in\left(f_{j}^{*}\right)_{j=1}^{\infty}$ a seqüência de funcionais biortogonais associada à base 
$\left(x_{n}\right)_{n=1}^{\infty}$. A seqüência $\left(f_{j}^{*}\right)_{j=1}^{\infty}$ é base de Schauder de $E^{*}$ se, e somente se, a base $\left(x_{n}\right)_{n=1}^{\infty}$ é contrátil.

Teorema 0.17 (L-T, pág. 9) Sejam E um espaço de Banach e $\left(x_{n}\right)_{n=1}^{\infty}$ uma base de Schauder de E. O espaço E é reflexivo se, e somente se, $\left(x_{n}\right)_{n=1}^{\infty}$ é contrátil e limitadamente completa.

Definição 0.18 Sejam $E$ um espaço normado e $\left(x_{n}\right)_{n=1}^{\infty}$ uma seqüência de $E$. Dizemos que $\left(x_{n}\right)_{n=1}^{\infty}$ converge fracamente para $x \in E$, se $\lim _{n \rightarrow+\infty} x^{*}\left(x_{n}\right)=x^{*}(x)$, $\forall x^{*} \in E^{*}$. Escrevemos então, $x_{n} \stackrel{w}{\longrightarrow} x$.

Definição 0.19 Uma seqüência infinita $\left(G_{n}\right)_{n=1}^{\infty}, G_{n} \neq\{0\}$, de subespaços (não necessariamente fechados) de um espaço de Banach $E$, é chamada de decomposição (ou uma base de subespaços) de $E$, se $\forall x \in E$ existe uma única seqüência $\left(y_{n}\right)_{n=1}^{\infty}$ com $y_{n} \in G_{n}$, tal que $x=\sum_{n=1}^{\infty} y_{n}$.

Definição 0.20 Seja $\left(G_{n}\right)_{n=1}^{\infty}$ uma decomposição do espaço de Banach E. A seqüência das projeções $\left(P_{n}\right)_{n=1}^{\infty}$ em $E$, definidas por $P_{n}(x)=y_{n}, x=\sum_{i=1}^{\infty} y_{n} \in E$, onde $y_{n} \in G_{n}, \forall n \in \mathbb{N}$, é chamada de seqüência de projeções coordenada associada à decomposição $\left(G_{n}\right)_{n=1}^{\infty}$.

Definição 0.21 Uma decomposição $\left(G_{n}\right)_{n=1}^{\infty}$ de um espaço de Banach $E$ é uma decomposição de Schauder (ou uma base de Schauder de subespaços) de $E$, se toda. projeção coordenada $P_{n}$ em $E$ é limitada.

Teorema 0.22 (S, pág. 489) Uma decomposição $\left(G_{n}\right)_{n=1}^{\infty}$ de um espaço de $B a$ nach E é uma decomposição de Schauder se, e somente se, $G_{n}$ é fechado, $\forall n \in \mathbb{N}$.

Teorema 0.23 (S, pág. 502) Sejam E um espaço de Banach e $\left(G_{n}\right)_{n=1}^{\infty}$ uma seqüencia de subespaços fechados de $E, \operatorname{com} G_{n} \neq\{0\}, \forall n \in \mathbb{N} e\left[\bigcup_{n=1}^{\infty} G_{n}\right]=E$. Então as seguintes afirmações são equivalentes: 
(a) $\left(G_{n}\right)_{n=1}^{\infty}$ é uma decomposição de Schauder de E;

(b) existe uma constante $C$, com $1 \leq C<\infty$, tal que $\left\|\sum_{i=1}^{n} y_{i}\right\| \leq C\left\|\sum_{i=1}^{n+m} y_{i}\right\|$, $\forall m, n \in \mathbb{N}$ e $\forall y_{i} \in G_{i}(i=1, \ldots, n+m)$.

Definição 0.24 Seja $X$ um espaço de Banach. Um operador $T: X \rightarrow X$ é estritamente singular, se a restrição de $T$ a qualquer subespaço fechado de $X$ de dimensão infinita não é um isomorfismo.

Proposição 0.25 (L-T, pág. 76) (a) A soma de dois operadores estritamente singulares é um operador estritamente singular.

(b) A composição de um operador estritamente singular e um operador limitado é um operador estritamente singular.

Definição 0.26 Sejam $X$ e $Y$ espaços de Banach, $T: X \rightarrow Y$ um operador linear limitado com $T(X)$ fechado, $\alpha(T)=\operatorname{dim} \operatorname{ker} T$ e $\beta(T)=\operatorname{dim} Y / T(X)$ (espaço quociente de $Y$ por $T(X))$. Se $\alpha(T)<\infty$ ou $\beta(T)<\infty$, definimos o índice de $T$ por $i(T)=\alpha(T)-\beta(T)$.

Definição 0.27 Sejam $X$ e $Y$ espaços de Banach e $T: X \rightarrow Y$ um operador linear limitado. Se $\alpha(T)$ e $\beta(T)$ são ambos finitos (logo $i(T)$ é definido e é finito), então $T$ é chamado de operador de Fredholm.

Proposição 0.28 (L-T, pág. 77) Sejam $X$ e $Y$ espaços de Banach, $T_{1}: X \rightarrow Y$ e $T_{2}: Y \rightarrow Z$ operadores de Fredholm. Então $T_{2} T_{1}$ é um operador de Fredholm e $i\left(T_{2} T_{1}\right)=i\left(T_{2}\right)+i\left(T_{1}\right)$.

Proposição 0.29 (L-T, pág. 79) Sejam $X$ e $Y$ espaços de Banach, $T: X \rightarrow Y$ um operador com imagem fechada, $S: X \rightarrow Y$ um operador estritamente singular. Então $\alpha(T+S)<\infty, T+S$ tem imagem fechada e $i(T+S)=i(T)$. 
Definição 0.30 Uma álgebra linear sobre o corpo $\mathbb{R}$ é um espaço vetorial $V$ sobre $\mathbb{R}$, com uma operação adicional dita multiplicação de vetores, que associa a cada. par de vetores $x$ e $y \in V$, o vetor $x y \in V$ e que satisfaz as seguintes propriedades:

(a) $x(y z)=(x y) z, \forall x, y, z, \in V$

(b) $x(y+z)=x y+x z$ e $(x+y) z=x z+y z, \forall x, y, z \in V$

(c) $\alpha(x y)=(\alpha x) y=x(\alpha y), \forall \alpha \in \mathbb{R}, \forall x, y \in V$.

Sejam $V$ e $V^{\prime}$ duas álgebras lineares. Uma transformação linear $\Phi: V \rightarrow V^{\prime}$ dizse um homomorfismo de álgebra, se satisfaz a seguinte condição: $\Phi(x y)=\Phi(x) \Phi(y)$, $\forall x, y \in V$.

Apresentaremos agora algumas definições e resultados que foram utilizados na. construção do espaço $X$ de Gowers e Maurey e no estudo de suas propriedades. A teoria apresentada pode ser encontrada em [G-M1] e [C].

Definição 0.31 Denotamos por $\mathcal{F}$ o conjunto das funções $f:[1,+\infty) \rightarrow[1,+\infty)$, satisfazendo as seguintes propriedades:

(1) $f(1)=1$ e $f(x)<x, \forall x>1$;

(2) $f$ é estritamente crescente e $\lim _{x \rightarrow+\infty} f(x)=+\infty$;

(3) $\lim _{x \rightarrow+\infty} x^{-q} f(x)=0, \forall q>0$;

(4) A função $\frac{x}{f(x)}$ é côncava;

(5) $f(x y) \leq f(x) f(y), \forall x, y \geq 1$.

Pelas observações 2.1 e 2.2 [C, pág. 39 a 43] as funções $f(x)=\log _{2}(x+1)$ e $\sqrt{f(x)}$ pertencem a $\mathcal{F}$. 
Definição 0.32 Seja $\mathcal{X}$ a classe dos espaços normados da forma $X=\left(c_{00},\|\cdot\|\right)$, tal que para cada espaço $X,\left(e_{i}\right)_{i=1}^{\infty}$ é uma base normalizada e monótona de $X$. Sejam $f \in \mathcal{F}$ e $X \in \mathcal{X}$, se para todo vetor $z \in X$ ocorrer a desigualdade:

$$
\|z\| \geq \sup \left\{f(N)^{-1} \sum_{i=1}^{N}\left\|E_{i}(z)\right\|: N \in \mathbb{N}, E_{1}<\cdots<E_{N}\right\},
$$

onde $E_{1}, \ldots, E_{N}$ são intervalos, dizemos que $X$ satisfaz uma $f$-estimativa inferior.

Observação 0.33 Se $X$ satisfaz uma $f$-estimativa inferior, então para todo vetor $z \in X$ e todo intervalo $E \subset \mathbb{N}$, teremos $\|E(z)\| \leq\|z\|$.

Definição 0.34 Sejam $X \in \mathcal{X}$ e um vetor $z \in X$, dizemos que $z$ é um $\ell_{1+}^{n}$-average com constante $C$, se $\|z\|=1$ e $z=\sum_{i=1}^{n} z_{i}$ para alguma seqüência $z_{1}<\cdots<z_{n}$ de vetores não nulos de $X$, tais que $\left\|z_{i}\right\| \leq C n^{-1}, 1 \leq i \leq n$.

Lema 0.35 (G-M1, pág. 857) Sejam $f \in \mathcal{F} \in X \in \mathcal{X}$ satisfazendo uma $f$-estimativa inferior. Então, para todo $n \in \mathbb{N}$ e $C>1$, qualquer subespaço de bloco $Y$ de $X$ contém um $\ell_{1+}^{n}$-average com constante $C$.

Definição 0.36 Sejam $f \in \mathcal{F}$ e $M_{f}: \mathbb{R} \rightarrow \mathbb{R}$ definida por $M_{f}(x)=f^{-1}\left(36 x^{2}\right)$. Dizemos que uma seqüência $z_{1}<z_{2}<\cdots<z_{N}$ é uma seqüência rapidamente crescente de $\ell_{1+}$-averages, ou RIS, para $f$ de comprimento $N$ com constante $1+\varepsilon$, se $z_{k}$ é um $\ell_{1+}^{n_{k}}$-average com constante $1+\varepsilon$ para cada $k, n_{1} \geq 2(1+\varepsilon) M_{f}\left(N / \varepsilon^{\prime}\right) / \varepsilon^{\prime} f^{\prime}(1)$ e $\frac{\varepsilon^{\prime}}{2} f\left(n_{k}\right)^{1 / 2} \geq\left|\operatorname{ran}\left(z_{k-1}\right)\right|$ para cada $k=2, \ldots, N$, onde $f^{\prime}(1)$ é a derivada à direita de $f$ em 1 e $\varepsilon^{\prime}$ é a notação usual para $\min \{\varepsilon, 1\}$.

Observação 0.37 Um ponto importante na definição de uma RIS é que os $n_{k}$ 's aumentam rapidamente; a velocidade dependerá do tamanho da ran dos $z_{j}$ 's anteriores. Algumas vezes será conveniente chamar um vetor de um vetor-RIS, se ele for um múltiplo não nulo da soma de uma RIS. 
Definição 0.38 Seja $g \in \mathcal{F}$. Um funcional $y^{*} \in X^{*}$ é chamado de uma $(M, g)$ forma se $\left\|y^{*}\right\| \leq 1$ e $y^{*}=\sum_{j=1}^{M} y_{j}^{*}$, para alguma seqüência $y_{1}^{*}<\cdots<y_{M}^{*}$ de funcionais sucessivos, tais que $\left\|y_{j}^{*}\right\| \leq g(M)^{-1}$, para cada $j, 1 \leq j \leq M$.

Definição 0.39 Seja $J \subset \mathbb{N}$ o conjunto denotado por $\left\{j_{1}, j_{2}, \ldots\right\}$, onde $j_{1}=$ $10^{10^{10^{400}}}, j_{2}=10^{10^{10^{4\left(j_{1}\right)^{2}}}}, \ldots, j_{n+1}=10^{10^{10^{4\left(j_{n}\right)^{2}}}}$.

Lema 0.40 (C, pág. 83) Para os elementos do conjunto J, temos

$$
\left(j_{2 k}\right)^{\frac{1}{40}} \geq \frac{40 M_{f}(10 k)}{f^{\prime}(1)}, \quad \forall k \in \mathbb{N},
$$

onde $f(x)=\log _{2}(x+1)$.

Definição 0.41 Sejam $K$ e $L$ subconjuntos de $J, K=\left\{j_{1}, j_{3}, j_{5}, \ldots\right\}$ e $L=$ $\left\{j_{2}, j_{4}, j_{6}, \ldots\right\}, Q$ o subconjunto de $c_{00}$ de todas as seqüencias cujas coordenadas são números racionais de módulo menor ou igual a um e $\sigma$ uma função injetora definida no conjunto das seqüências finitas de elementos sucessivos de $Q$ com imagem no conjunto $L$, tal que se $Z_{1}, \ldots, Z_{s}$ é uma qualquer dessas seqüências sucessivas, $S=\sigma\left(Z_{1}, \ldots, Z_{s}\right)$ e $Z=\sum_{i=1}^{s} Z_{i}$, então $\frac{1}{20} f\left(S^{\frac{1}{40}}\right)^{\frac{1}{2}} \geq|\operatorname{ran}(Z)|$.

Definição 0.42 Seja $X=\left(c_{00},\|\cdot\|\right)$ um espaço normado sobre as seqüências de suporte finito. Para todo $m \in \mathbb{N}$, definimos $A_{m}^{*}(X)$ como sendo o conjunto dos funcionais lineares da forma $f(m)^{-1} \sum_{i=1}^{m} f_{i}$ tais que

(1) $f_{1}<f_{2}<\cdots<f_{m}$

(2) $\left\|f_{i}\right\| \leq 1, i=1,2, \ldots, m$.

Definição 0.43 Seja $X=\left(c_{00},\|\cdot\|\right)$. Se $k \in \mathbb{N}$, seja $\Gamma_{k}^{X}$ o conjunto das seqüencias $g_{1}<g_{2}<\cdots<g_{k}$ tais que $g_{i} \in Q$, para cada $i, g_{1} \in A_{j_{2 k}}^{*}(X)$ e $g_{i+1} \in A_{\sigma\left(g_{1}, \ldots, g_{i}\right)}^{*}(X)$ para qualquer $i, 1 \leq i \leq k-1$. Estas seqüências são chamadas de seqüências especiais. 
Definição 0.44 Seja $X=\left(c_{00},\|\cdot\|\right)$, para todo $k \in \mathbb{N}$, definimos $B_{k}^{*}(X)$ como o conjunto dos funcionais lineares da forma $f(k)^{-\frac{1}{2}} \sum_{j=1}^{k} g_{j}$ tal que $\left(g_{1}, \ldots, g_{k}\right) \in \Gamma_{k}^{X}$. Estes funcionais são chamados de funcionais especiais.

Definição 0.45 (C, págs. 85 e 86) Seja $X_{0}=\left(c_{00},\|\cdot\|_{0}\right)$, onde $\|z\|_{0}=\|z\|_{\infty}$ e para $N \geq 0$ definimos:

$$
\begin{aligned}
\|z\|_{X_{N+1}}= & \|z\|_{X_{N}} \vee \sup \left\{f(n)^{-1} \sum_{i=1}^{n}\left\|E_{i}(z)\right\|_{X_{N}}: n \geq 2, E_{1}<\cdots<E_{n}\right\} \\
& \vee \sup \left\{|\bar{g}(E(z))|: k \in K, \bar{g} \in B_{k}^{*}\left(X_{N}\right), E \subset \mathbb{N}\right\},
\end{aligned}
$$

onde $E, E_{1}, \ldots, E_{n}$ são intervalos. A seqüência de normas $\left(\|z\|_{X_{N}}\right)_{N=0}^{\infty}$ é crescente e é limitada pela norma $\ell_{1}$, logo existe $\lim _{N \rightarrow+\infty}\|z\|_{X_{N}}$. Definimos então $\|z\|=$ $\lim _{N \rightarrow+\infty}\|z\|_{X_{N}}$ e o espaço normado $X=\left(c_{00},\|\cdot\|\right)$. Além disso, pela proposição 4.10 [C, pág. 87],

$$
\begin{aligned}
\|z\|= & \|z\|_{\infty} \vee \sup \left\{f(n)^{-1} \sum_{i=1}^{n}\left\|E_{i}(z)\right\|: n \geq 2, E_{1}<\cdots<E_{n}\right\} \\
& \vee \sup \left\{|\bar{g}(E(z))|: k \in K, \bar{g} \in B_{k}^{*}(X), E \subset \mathbb{N}\right\}
\end{aligned}
$$

é uma forma implícita para a norma de $z \in X=\left(c_{00},\|\cdot\|\right)$.

Definição $0.46 \mathrm{O}$ completado de $X=\left(c_{00},\|\cdot\|\right)$ é o espaço de Gowers e Maurey, denotado por $X_{G M}$ ou simplesmente $X$.

Observação 0.47 (G-M1, págs. 863 e 865) A seqüência $\left(e_{n}\right)_{n=1}^{\infty}$ é uma base normalizada do espaço $X$ de Gowers e Maurey. Além disso, o espaço $X$ satisfaz uma. $f$-estimativa inferior.

Lema 0.48 (G-M1, pág. 865) Sejam $N \in L, n \in[\log N, \exp N], \varepsilon>0$ e $z_{1}, \ldots$, $z_{n}$ uma RIS com constante $1+\varepsilon$. Entãa $\left\|\sum_{i=1}^{n} z_{i}\right\| \leq\left(1+\varepsilon+\varepsilon^{\prime}\right) n f(n)^{-1}$. 
Lema 0.49 (G-M1, pág. 866) Sejam $k \in K, s_{1}^{*}, \ldots, s_{k}^{*}$ uma seqüência especial de comprimento $k$, onde cada $s_{i}^{*}$ é uma $\left(M_{i}, f\right)$-forma. Seja $z_{1}, \ldots, z_{k}$ uma seqüência de vetores sucessivos tais que todo $z_{i}$ é um vetor RIS normalizado de comprimento $M_{i}$ e constante $1+\varepsilon / 4, \varepsilon=\frac{1}{10}$. Assuma que $\left|\left(\sum_{i=1}^{k} s_{i}^{*}\right)\left(\sum_{i=1}^{k} E\left(z_{i}\right)\right)\right| \leq 2$ para todo intervalo E. Então $\left\|\sum_{i=1}^{k} z_{i}\right\| \leq(1+2 \varepsilon) k / f(k)$.

Proposição 0.50 (C, pág. 110) O espaço $X$ de Gowers e Maurey é um espaço reflexivo.

Definição 0.51 Um espaço de Banach $X$ de dimensão infinita é H.I. (hereditariamente indecomponível) se nenhum subespaço fechado de $X$ de dimensão infinita, pode ser decomposto como soma direta topológica de dois subespaços fechados de $X$ de dimensão infinita.

Teorema 0.52 (G-M1, pág. 867) O espaço de Banach X de Gowers e Maurey é hereditariamente indecomponível.

Lema 0.53 (C, pág. 121) Um espaço de Banach $X$ é hereditariamente indecomponível se, e somente se, para quaisquer $Y$ e $Z$ subespaços fechados de dimensão infinita de $X$ e para qualquer $\varepsilon>0$, existem $y \in Y e z \in Z,\|y\|=\|z\|=1 e$ $\|y-z\|<\varepsilon$. 


\section{Capítulo 1}

\section{O espaço $W$}

Neste capítulo construiremos o espaço de Banach W. Como já foi dito na introdução, mostraremos que este espaço e o espaço $X \oplus \mathrm{W}$ formam o contra-exemplo para o problema de Schroeder-Bernstein. Para facilitar a leitura, faremos agora um esboço da estrutura deste capítulo.

Inicialmente apresentaremos duas propriedades importantes do espaço $X$ de Gowers e Maurey. A primeira (Lema 1.1) se refere a uma desigualdade válida para a norma de vetores de $X$, que são soma finita de vetores sucessivos de $X$, e será utilizada no capítulo seguinte.

A segunda propriedade (Lema 1.7) garantirá a existência de uma seqüência normalizada de vetores sucessivos $\left(x_{i}\right)_{i=1}^{\infty} \subset X$ tal que, para todo $T \in B(X)$, existirá $\lambda \in \mathbb{R} \operatorname{com} \lim _{i \rightarrow+\infty} T\left(x_{i}\right)-\lambda x_{i}=0$. Esta seqüência e a propriedade do limite, terão um papel importante na construção da solução para o problema de Schoreder-Bernstein.

Para a construção de $\mathbf{W}$, utilizaremos o espaço vetorial V, de todas as seqüencias finitas de vetores pertencentes a $X \oplus_{\infty} X$.

A partir do Lema 1.8, definiremos uma seqüência $\left(x_{i}^{*}\right)_{i=1}^{\infty}$ de funcionais lineares associados à seqüência $\left(x_{i}\right)_{i=1}^{\infty}$, que nos ajudarão a definir uma norma $\|\cdot\|_{v} \mathrm{em} V$. 
O espaço de Banach W será o completado de $\mathrm{V}$ com a norma $\|\cdot\|_{v}$.

No final, concluiremos que a seqüência $\left(X_{i}\right)_{i=1}^{\infty}$ de cópias isométricas de $X$ é uma decomposição de Schauder de W (Lema 1.17 e Observação 1.20). Além disso, pelo Lema 1.22 , provaremos $\mathrm{W} \stackrel{c}{\hookrightarrow} X \oplus \mathrm{W}$ e $X \oplus \mathrm{W} \stackrel{c}{\hookrightarrow} \mathrm{W}$ e também que $(X \oplus \mathrm{W})^{3} \sim$ $(X \oplus \mathbf{W})$ e $(X \oplus \mathbf{W})^{2} \sim \mathbf{W}$.

A teoria apresentada neste capítulo pode ser encontrada em [G-M1] e [G].

Lema 1.1 Sejam X o espaço de Gowers e Maurey e $z_{1}<z_{2}<\cdots<z_{n}$ uma seqüência de vetores sucessivos de $X$. Então vale a desigualdade

$$
\left\|\sum_{i=1}^{n} z_{i}\right\| \geq\left(\log _{2}(n+1)\right)^{-1} \sum_{i=1}^{n}\left\|z_{i}\right\| .
$$

Demonstração: Sejam $z=\sum_{i=1}^{n} z_{i}, f(x)=\log _{2}(x+1) \in \mathcal{F}$ (veja a Definição $0.31)$ e $E_{i}(z)=\operatorname{ran}\left(z_{i}\right)$. Como $X$ satisfaz uma $f$-estimativa inferior (veja a Definição 0.32 e Observação 0.47$)$ e $E_{i}(z)<E_{i+1}(z), i=1, \ldots, n-1$, teremos $\|z\| \geq(f(n))^{-1} \sum_{i=1}^{n}\left\|E_{i}(z)\right\|=\left(\log _{2}(n+1)\right)^{-1} \sum_{i=1}^{n}\left\|z_{i}\right\|$.

Definição 1.2 Sejam $X$ o espaço de Gowers e Maurey, $T \in B(X)$ e $y \in X$, tais que $y$ e $T(y)$ têm suporte finito. Definimos $I(y)$ como sendo o menor intervalo que contém $\operatorname{supp}(y)$ e supp $(T(y))$.

Lema 1.3 Sejam $X$ o espaço de Gowers e Maurey, $T \in B(X),\left(y_{n}\right)_{n=1}^{\infty}$ uma seqüência contida em $X$ de $\ell_{1+}^{n}$-averages sucessivos com constante $1+\frac{\varepsilon}{4}, \varepsilon=\frac{1}{10}$, para todo $n \in \mathbb{N}$, e também $\delta>0$. Então existe uma subseqüência $\left(y_{n_{k}}\right)_{k=1}^{\infty}$ de $\left(y_{n}\right)_{n=1}^{\infty}$, um operador limitado $\bar{T}: Y \rightarrow X$ onde $Y=\left[\left(y_{n_{k}}\right)_{k=1}^{\infty}\right]$, tal que se $T^{\prime}: Y \rightarrow X$ é a restrição do operador $T$ ao subespaço $Y$, então $\left\|\bar{T}-T^{\prime}\right\|<\delta$. Além disso, podemos obter uma subseqüência $\left(y_{n_{k_{i}}}\right)_{i=1}^{\infty}$ de $\left(y_{n_{k}}\right)_{k=1}^{\infty}$ tal que, se $I\left(y_{n_{k_{i}}}\right)$ é o menor intervalo que contém $\operatorname{supp}\left(y_{n_{k_{i}}}\right)$ e $\operatorname{supp}\left(\bar{T}\left(y_{n_{k_{i}}}\right)\right.$ então, $I\left(y_{n_{k_{i}}}\right)<I\left(y_{n_{k_{i+1}}}\right), \forall i \in \mathbb{N}$. 
Demonstração: Mostremos inicialmente que $y_{n} \stackrel{w}{\longrightarrow} 0$. A seqüência de $\ell_{1+}^{n}$-averages $\left(y_{n}\right)_{n=1}^{\infty}$ (veja Definição 0.34), é uma seqüência básica de $X$; pois $y_{n} \neq 0, \forall n \in$ $\mathbb{N}$, e se $\left(a_{n}\right)_{n=1}^{\infty}$ é uma seqüência de escalares e $m, n$ são inteiros com $m<n$, teremos $\left\|\sum_{i=1}^{m} a_{i} y_{i}\right\| \leq\left\|\sum_{i=1}^{n} a_{i} y_{i}\right\|$, pois se considerarmos $\sum_{i=1}^{n} a_{i} y_{i}=x \in X$ e $E(x)=$ $\sum_{i=1}^{m} a_{i} y_{i}$; então pela Observação $0.33,\|E(x)\| \leq\|x\|$, pois $X$ satisfaz uma $f$-estimativa. inferior.

Assim, $\left(y_{n}\right)_{n=1}^{\infty}$ é base de Schauder normalizada do subespaço fechado $Z=$ $\left[\left(y_{n}\right)_{n=1}^{\infty}\right]$ de $X$. Pela Proposição $0.50, X$ é um espaço reflexivo, logo pela Proposição $0.12 Z$ é reflexivo, então pelo Teorema 0.17 a base $\left(y_{n}\right)_{n=1}^{\infty}$ é shrinking.

Considerando os funcionais biortogonais $f_{n}^{*}: Z^{*} \rightarrow \mathbb{R}$, associados à base $\left(y_{n}\right)_{n=1}^{\infty}$ (veja Definição 0.6) temos pela Proposição 0.16 que $\left(f_{n}^{*}\right)_{n=1}^{\infty}$ é base de $Z^{*}$. Em particular, temos $f_{n}^{*}\left(y_{n}\right)=1, \operatorname{logo}\left\|f_{n}^{*}\right\| \geq 1, \forall n \in \mathbb{N}$.

Seja $z^{*} \in Z^{*}$, existe uma única seqüência de escalares $\left(b_{n}\right)_{n=1}^{\infty}$ tal que $z^{*}=$ $\sum_{n=1}^{\infty} b_{n} f_{n}^{*}, \operatorname{logo} \lim _{n \rightarrow+\infty}\left\|b_{n} f_{n}^{*}\right\|=0$, em particular, $\lim _{n \rightarrow+\infty}\left|b_{n}\right|=0$, mas $z^{*}\left(y_{n}\right)=$ $\sum_{i=1}^{\infty} b_{i} f_{i}^{*}\left(y_{n}\right)=b_{n}$ e portanto, $\lim _{n \rightarrow+\infty} z^{*}\left(y_{n}\right)=\lim _{n \rightarrow+\infty} b_{n}=0$, logo para qualquer $x^{*} \in X^{*}$, teremos $\lim _{n \rightarrow+\infty} x^{*}\left(y_{n}\right)=\left.\lim _{n \rightarrow+\infty} x^{*}\right|_{Z}\left(y_{n}\right)=0$.

Olhemos agora para a seqüência de vetores $\left(T\left(y_{n}\right)\right)_{n=1}^{\infty}$. Como $y_{n} \stackrel{w}{\longrightarrow} 0$ e $T \in$ $B(X)$, segue que $T\left(y_{n}\right) \stackrel{w}{\longrightarrow} 0$. Considerando os funcionais biortogonais $e_{j}^{*}$ associados à base $\left(e_{j}\right)_{j=1}^{\infty}$ de $X$, temos $\lim _{n \rightarrow+\infty} e_{j}^{*}\left(T\left(y_{n}\right)\right)=0, \forall j \in \mathbb{N}$. Escrevemos $e_{j}^{*}\left(T\left(y_{n}\right)\right)=\lambda_{j}^{n}$ (a $j$-ésima coordenada do vetor $T\left(y_{n}\right)$ na base $\left.\left(e_{j}\right)_{j=1}^{\infty}\right), \log$ $\lim _{n \rightarrow+\infty} \lambda_{j}^{n}=0$ para todo $j \in \mathbb{N}$.

Mostremos agora que dado $\delta>0$ é possível determinar uma subseqüência de $\left(T\left(y_{n}\right)\right)_{n=1}^{\infty},\left(T\left(y_{n_{k}}\right)\right)_{k=1}^{\infty}$ e uma seqüência $z_{k}$ de blocos finitos contida em $X$ e com 
$\lim _{k \rightarrow+\infty} \min \operatorname{supp}\left(z_{k}\right)=+\infty$, em relação à base $\left(e_{j}\right)_{j=1}^{\infty}$ de $X$ e tal que $\sum_{k=1}^{\infty} \| T\left(y_{n_{k}}\right)-$ $z_{k} \|<\delta$.

De fato, existe $n_{1} \in \mathbb{N}$ tal que $T\left(y_{n_{1}}\right)=\sum_{j=1}^{\infty} \lambda_{j}^{n_{1}} e_{j}$ tem $\left\|\lambda_{1}^{n_{1}} e_{1}\right\|<\frac{\delta}{4}$ e existe $N_{1} \in \mathbb{N}, N_{1}>1$, tal que $\left\|\sum_{j=N_{1}+1}^{\infty} \lambda_{j}^{n_{1}} e_{j}\right\|<\frac{\delta}{4}$. Consideremos $z_{1}=\sum_{j=2}^{N_{1}} \lambda_{j}^{n_{1}} e_{j}, \log \mathrm{o}$ $\left\|T\left(y_{n_{1}}\right)-z_{1}\right\|=\left\|\lambda_{1}^{n_{1}} e_{1}+\sum_{j=N_{1}+1}^{\infty} \lambda_{j}^{n_{1}} e_{j}\right\|<\frac{\delta}{4}+\frac{\delta}{4}=\frac{\delta}{2}$ e $\min \operatorname{supp}\left(z_{1}\right)=2$.

Existe $n_{2}>n_{1} \in \mathbb{N}$ tal que $T\left(y_{n_{2}}\right)=\sum_{j=1}^{\infty} \lambda_{j}^{n_{2}} e_{j}$ tem $\left\|\lambda_{1}^{n_{2}} e_{1}+\lambda_{2}^{n_{2}} e_{2}\right\|<\frac{\delta}{8}$ e existe $N_{2} \in \mathbb{N}, N_{2}>N_{1}$ tal que $\left\|\sum_{j=N_{2}+1}^{\infty} \lambda_{j}^{n_{2}} e_{j}\right\|<\frac{\delta}{8}$. Tomemos $z_{2}=\sum_{j=3}^{N_{2}} \lambda_{j}^{n_{2}} e_{j}, \log \mathrm{o}$ $\left\|T\left(y_{n_{2}}\right)-z_{2}\right\|=\left\|\lambda_{1}^{n_{2}} e_{1}+\lambda_{2}^{n_{2}} e_{2}+\sum_{j=N_{2}+1}^{\infty} \lambda_{j}^{n_{2}} e_{j}\right\|<\frac{\delta}{8}+\frac{\delta}{8}=\frac{\delta}{4}$ e também min supp $\left(z_{2}\right)=3$.

Vamos provar agora por indução que para todo $k \in \mathbb{N}$, existem $T\left(y_{n_{k}}\right)$ e $z_{k}$, tais que $\left\|T\left(y_{n_{k}}\right)-z_{k}\right\|<\frac{\delta}{2^{k}}$ e min $\operatorname{supp}\left(z_{k}\right)=k$.

Suponhamos que para $k>2$ tenhamos $T\left(y_{n_{k}}\right)=\sum_{j=1}^{\infty} \lambda_{j}^{n_{k}} e_{j}$ e $z_{k}=\sum_{j=k+1}^{N_{k}} \lambda_{j}^{n_{k}} e_{j}$. Existirá $n_{k+1} \in \mathbb{N}, n_{k+1}>n_{k}$, tal que $T\left(y_{n_{k+1}}\right)=\sum_{j=1}^{\infty} \lambda_{j}^{n_{k+1}} e_{j} \in X$ tem $\| \lambda_{1}^{n_{k+1}} e_{1}+\cdots$ $+\lambda_{k+1}^{n_{k+1}} e_{k+1} \|<\frac{\delta}{2^{k+2}}$ e existirá $N_{k+1} \in \mathbb{N}, N_{k+1}>N_{k}$ tal que $\left\|\sum_{j=N_{k+1}+1}^{\infty} \lambda_{j}^{n_{k+1}} e_{j}\right\|<$ $\frac{\delta}{2^{k+2}}$.

Consideremos $z_{k+1}=\sum_{j=k+2}^{N_{k+1}} \lambda_{j}^{n_{k+1}} e_{j}, \operatorname{logo}\left\|T\left(y_{n_{k+1}}\right)-z_{k+1}\right\|=\|\left(\lambda_{1}^{n_{k+1}} e_{1}+\cdots\right.$ $\left.\cdots+\lambda_{k+1}^{n_{k+1}} e_{k+1}\right)+\sum_{j=N_{k+1}+1}^{\infty} \lambda_{j}^{n_{k+1}} e_{j} \|<\frac{\delta}{2^{k+2}}+\frac{\delta}{2^{k+2}}=\frac{\delta}{2^{k+1}}$ e minsupp $\left(z_{k+1}\right)=k+2$.

Concluímos que $\sum_{k=1}^{\infty}\left\|T\left(y_{n_{k}}\right)-z_{k}\right\|<\left(\sum_{k=1}^{\infty} \frac{1}{2^{k}}\right) \delta<\delta$, além disso $\lim _{k \rightarrow+\infty} \min \operatorname{supp}$ $\left(z_{k}\right)=\lim _{k \rightarrow+\infty} k+1=+\infty$.

Seja. $Y=\left[\left(y_{n_{k}}\right)_{k=1}^{\infty}\right], Y<X$, conforme verificamos anteriormente para o subespaço $Z$, a seqüência $\left(y_{n_{k}}\right)_{k=1}^{\infty}$ é base de Schauder normalizada de $Y$. 
Definimos uma aplicação $\bar{T}: Y \rightarrow X$ por $\bar{T}\left(y_{n_{k}}\right)=z_{k}$, isto é, $\bar{T}\left(\sum_{k=1}^{\infty} a_{k} y_{n_{k}}\right)=$ $\sum_{k=1}^{\infty} a_{k} z_{k}$. Mostremos que $\bar{T}$ está bem definida. Antes porém, observemos que $\left|a_{k}\right|=$ $\left\|a_{k} y_{n_{k}}\right\| \leq\left\|\sum_{i=k}^{l} a_{i} y_{n_{i}}\right\|$ para todo $k, l \in \mathbb{N}$, com $l>k$; pois $X$ satisfaz uma $f$ estimativa inferior.

Consideremos a seqüencia das somas parciais $S_{p}=\sum_{k=1}^{p} a_{k} z_{k}$, se $p, l \in \mathbb{N}$ com $l>p$ teremos:

$$
\begin{aligned}
\left\|S_{l}-S_{p}\right\| & =\left\|\sum_{k=p+1}^{l} a_{k} z_{k}\right\| \leq\left\|\sum_{k=p+1}^{l} a_{k}\left(z_{k}-T\left(y_{n_{k}}\right)\right)\right\|+\left\|\sum_{k=p+1}^{l} a_{k} T\left(y_{n_{k}}\right)\right\| \\
& \leq \sum_{k=p+1}^{l}\left|a_{k}\right|\left\|T\left(y_{n_{k}}\right)-z_{k}\right\|+\left\|\sum_{k=p+1}^{l} a_{k} T\left(y_{n_{k}}\right)\right\| \\
& \leq\left\|\sum_{k=p+1}^{l} a_{k} y_{n_{k}}\right\| \delta+\left\|\sum_{k=p+1}^{l} a_{k} T\left(y_{n_{k}}\right)\right\| .
\end{aligned}
$$

Como as séries $\sum_{k=1}^{\infty} a_{k} y_{n_{k}}$ e $\sum_{k=1}^{\infty} a_{k} T\left(y_{n_{k}}\right)$ convergem, então $\left\|S_{l}-S_{p}\right\| \rightarrow 0$ quando $l, p \rightarrow+\infty$, isto é, $\sum_{k=1}^{\infty} a_{k} z_{k}$ converge. Obviamente $\bar{T}$ é linear.

Falta mostrar que $\bar{T}$ é limitada. Consideremos para isso o operador limitado $T^{\prime}$ : $Y \rightarrow X$ definido por $T^{\prime}\left(\sum_{k=1}^{\infty} a_{k} y_{n_{k}}\right)=T\left(\sum_{k=1}^{\infty} a_{k} y_{n_{k}}\right)$. Seja $y=\sum_{k=1}^{\infty} a_{k} y_{n_{k}} \operatorname{com}\|y\|=1$. Como $X$ satisfaz uma $f$-estimativa inferior, temos $\left|a_{k}\right|=\left\|a_{k} y_{n_{k}}\right\| \leq\left\|\sum_{k=1}^{\infty} a_{k} y_{n_{k}}\right\|=1$. Logo, $\left\|\left(\bar{T}-T^{\prime}\right)(y)\right\|=\left\|\left(\bar{T}-T^{\prime}\right)\left(\sum_{k=1}^{\infty} a_{k} y_{n_{k}}\right)\right\|=\left\|\sum_{k=1}^{\infty} a_{k}\left(T\left(y_{n_{k}}\right)-z_{k}\right)\right\| \leq \sum_{k=1}^{\infty}\left|a_{k}\right| \| T\left(y_{n_{k}}\right)$ $-z_{k}\left\|\leq \sum_{k=1}^{\infty}\right\| T\left(y_{n_{k}}\right)-z_{k} \|<\delta$. Portanto, $\bar{T}$ é limitada e $\left\|\bar{T}-T^{\prime}\right\|<\delta$.

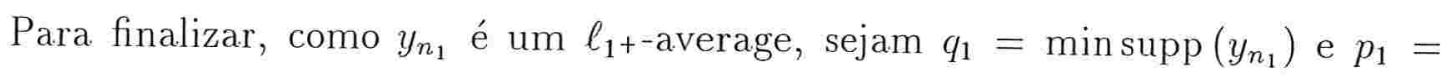
máx supp $\left(y_{n_{1}}\right)$. Como $\bar{T}\left(y_{n_{1}}\right)$ é um bloco finito, sejam $s_{1}=\min \operatorname{supp}\left(\bar{T}\left(y_{n_{1}}\right)\right)$ e $r_{1}=$ máx supp $\left(\bar{T}\left(y_{n_{1}}\right)\right)$. Tomemos $L_{1}=\min \left\{q_{1}, s_{1}\right\}$ e $M_{1}=\operatorname{máx}\left\{p_{1}, r_{1}\right\}$ e consideremos $I\left(y_{n_{1}}\right)=I\left(y_{n_{k_{1}}}\right)=\left\{L_{1}, L_{1}+1, \ldots, M_{1}\right\}, \operatorname{logo} I\left(y_{n_{1}}\right)$ é o menor intervalo que contém $\operatorname{supp}\left(y_{n_{1}}\right) \operatorname{esupp}\left(\bar{T}\left(y_{n_{1}}\right)\right)$. 
Agora, como $\lim _{k \rightarrow+\infty} \min \operatorname{supp}\left(\bar{T}\left(y_{n_{k}}\right)\right)=+\infty$ e $\left(y_{n_{k}}\right)_{k=1}^{\infty}$ é uma seqüência crescente de $\ell_{1+}^{n_{k}}$-averages, existe $k_{2} \in \mathbb{N}$ tal que $\min \operatorname{supp}\left(\bar{T}\left(y_{n_{k_{2}}}\right)\right)=s_{2}>M_{1} \mathrm{e}$ $\min \operatorname{supp}\left(y_{n_{k_{2}}}\right)=q_{2}>M_{1}$. Sejam máx $\operatorname{supp}\left(\bar{T}\left(y_{n_{k_{2}}}\right)\right)=r_{2}$ e máx $\operatorname{supp}\left(y_{n_{k_{2}}}\right)=p_{2}$. Tomemos $L_{2}=\min \left\{s_{2}, q_{2}\right\}$ e $M_{2}=\operatorname{máx}\left\{p_{2}, r_{2}\right\}$ e consideremos $I\left(y_{n_{k_{2}}}\right)=\left\{L_{2}, L_{2}+\right.$ $\left.1, \ldots, M_{2}\right\}$.

Continuando desta forma obtemos uma subseqüência $\left(y_{n_{k_{i}}}\right)_{i=1}^{\infty}$ de $\left(y_{n_{k}}\right)_{k=1}^{\infty}$ contida em $Y$ tal que $I\left(y_{n_{k_{i}}}\right)<I\left(y_{n_{k_{i+1}}}\right), \forall i \in \mathbb{N}$.

Lema 1.4 Sejam $X$ o espaço de Gowers e Maurey, $T: X \rightarrow X$ um operador limitado, $\delta>0, k \in K, y^{*}=\sum_{i=1}^{M} y_{i}^{*} u m a(M, f)$-forma, $M \in L e z \in X, z=\sum_{i=1}^{M} z_{i}$, um vetor RIS normalizado de comprimento $M$ satisfazendo ran $\left(y_{i}^{*}\right) \subset I\left(z_{i}\right), 1 \leq$ $i \leq M, y^{*}(z)=0$ e $y^{*}(T(z))>\delta$. Então, existe uma $(M, f)$-forma $s^{*} \in Q$ tal que $\left|s^{*}(z)\right| \leq k^{-2}, \operatorname{ran}\left(s^{*}\right) \subset I(z) e s^{*}(T(z))>\frac{\delta}{2}$.

Demonstração: Temos por hipótese (veja Definição 0.36, Observação 0.37 e Definição 0.38) que $\left\|y^{*}\right\| \leq 1,\left\|y_{i}^{*}\right\| \leq f(M)^{-1}, y_{1}^{*}<y_{2}^{*}<\cdots<y_{M}^{*}$. Além disso, $z_{1}<\cdots<z_{M}$ e também ran $\left(y_{i}^{*}\right) \subset I\left(z_{i}\right)$.

Sejam $n_{i}=\operatorname{máx}\left\{\operatorname{máx} \operatorname{ran}\left(z_{i}\right)\right.$, máx $\left.\operatorname{ran}\left(y_{i}^{*}\right)\right\}, p_{i}=\min \left\{\min \operatorname{ran}\left(z_{i}\right), \min \operatorname{ran}\left(y_{i}^{*}\right)\right\}$, $1 \leq i \leq M, N=\operatorname{máx}\left\{n_{i}-p_{i}: 1 \leq i \leq M\right\}$ e coloquemos $y_{i}^{*}=\left(c_{p_{i}}^{i}, c_{p_{i}+1}^{i}, \ldots, c_{n_{i}}^{i}\right)$, onde alguns desses $c_{j}^{i}$ 's são nulos.

Seja $\varepsilon \in \mathbb{R}$, definindo:

(1) $\alpha=f(M)^{-1}$;

(2) $\beta(\varepsilon)=\varepsilon N+\alpha$;

(3) $\gamma(\varepsilon)=\varepsilon N M+1$; 
(4) $\theta(\varepsilon)=\left(1-\frac{\alpha}{\varepsilon N+\alpha}\right)(\varepsilon N M+1)+\varepsilon N M$;

(5) $\mu(\varepsilon)=\frac{\alpha}{\beta(\varepsilon) \gamma(\varepsilon)}$

Como $\lim _{\varepsilon \rightarrow 0^{+}} \theta(\varepsilon)=0, \mu(0)=1, \mu(\varepsilon) \leq 1, \forall \varepsilon \in \mathbb{R}$ e $\mu$ é contínua, pelo teorema do valor intermediário do cálculo, existe $\varepsilon \in \mathbb{R}$ tal que:

(6) $\theta(\varepsilon)<\min \left\{k^{-2}, \frac{\delta}{2\|T\|}\right\}$, onde $k \in K$ (veja Definições 0.39 e 0.41$)$;

(7) $\mu(\varepsilon)$ é um número racional.

Seja $r_{i}^{*}=\left(b_{p_{i}}^{i}, b_{p_{i}+1}^{i}, \ldots, b_{n_{i}}^{i}\right) \in Q$ (veja Definição 0.41$)$, onde os $b_{j}^{i}$ 's são não nulos, $1 \leq i \leq M$, com $\left|c_{j}^{i}-b_{j}^{i}\right| \leq \varepsilon, p_{i} \leq j \leq n_{i}$.

Seja $t \in X,\|t\| \leq 1, \operatorname{com} t=\sum_{i=1}^{\infty} a_{i} e_{i}, \operatorname{temos}\left|r_{i}^{*}(t)-y_{i}^{*}(t)\right|=\left|\sum_{j=p_{i}}^{n_{i}} a_{j}\left(c_{j}^{i}-b_{j}^{i}\right)\right| \leq$ $\sum_{j=p_{i}}^{n_{i}}\left|a_{j}\left(c_{j}^{i}-b_{j}^{i}\right)\right| \leq \varepsilon\left(n_{i}-p_{i}\right)$ máx $\left\{\left|a_{j}\right|: p_{i} \leq j \leq n_{i}\right\} \leq \varepsilon N$.

$\operatorname{Logo}\left|r_{i}^{*}(t)\right| \leq\left|r_{i}^{*}(t)-y_{i}^{*}(t)\right|+\left|y_{i}^{*}(t)\right| \leq \varepsilon N+\left\|y_{i}^{*}\right\|\|t\| \leq \varepsilon N+f(M)^{-1}=\beta(\varepsilon) \mathrm{e}$ $\operatorname{assim}\left\|r_{i}^{*}\right\| \leq \beta(\varepsilon)$, isto é, $\left\|\frac{r_{i}^{*}}{\beta(\varepsilon)}\right\| \leq 1,1 \leq i \leq M$.

Consideremos agora, $r^{*}=\sum_{i=1}^{M} r_{i}^{*}$. Seja $t \in X,\|t\| \leq 1 . \operatorname{Logo},\left|r^{*}(t)-y^{*}(t)\right| \leq$ $\sum_{i=1}^{M}\left|r_{i}^{*}(t)-y_{i}^{*}(t)\right| \leq \varepsilon N M$

Portanto, $\left|r^{*}(t)\right| \leq\left|r^{*}(t)-y^{*}(t)\right|+\left|y^{*}(t)\right| \leq \varepsilon N M+\left\|y^{*}\right\|\|t\| \leq \varepsilon N M+1=\gamma(\varepsilon)$ e $\operatorname{assim}\left\|r^{*}\right\| \leq \gamma(\varepsilon)$, isto é, $\left\|\frac{r^{*}}{\gamma(\varepsilon)}\right\| \leq 1$

Vamos finalmente definir $s^{*}$ pondo $s^{*}=\frac{\alpha}{\beta(\varepsilon) \gamma(\varepsilon)} r^{*}$, ou seja, $s^{*}=\sum_{i=1}^{M} \frac{\alpha}{\beta(\varepsilon) \gamma(\varepsilon)} r_{i}^{*}$. Portanto, $s^{*}$ tem as seguintes propriedades:

(a) $\left\|\frac{\alpha}{\beta(\varepsilon) \gamma(\varepsilon)} r_{i}^{*}\right\|=\frac{\alpha}{\gamma(\varepsilon)}\left\|\frac{r_{i}^{*}}{\beta(\varepsilon)}\right\| \leq \alpha 1=f(M)^{-1}$;

(b) $\left\|s^{*}\right\|=\left\|\frac{\alpha}{\beta(\varepsilon) \gamma(\varepsilon)} r^{*}\right\|=\frac{\alpha}{\beta(\varepsilon)}\left\|\frac{r^{*}}{\gamma(\varepsilon)}\right\| \leq 1$; 
(c) $r_{1}^{*}<r_{2}^{*}<\cdots<r_{M}^{*}$ e $\operatorname{ran}\left(s^{*}\right) \subset I(z)$;

(d) $\left|s^{*}(z)\right|=\left|s^{*}(z)-y^{*}(z)\right|=\left|\frac{\alpha}{\beta(\varepsilon) \gamma(\varepsilon)} r^{*}(z)-y^{*}(z)\right| \leq\left|\frac{\alpha}{\beta(\varepsilon) \gamma(\varepsilon)} r^{*}(z)-r^{*}(z)\right|$ $+\left|r^{*}(z)-y^{*}(z)\right|=\left|\left(\frac{\alpha}{\beta(\varepsilon) \gamma(\varepsilon)}-1\right) r^{*}(z)\right|+\left|r^{*}(z)-y^{*}(z)\right|$ $\leq\left(1-\frac{\alpha}{\beta(\varepsilon) \gamma(\varepsilon)}\right)\left\|r^{*}\right\|\|z\|+\varepsilon N M \leq\left(1-\frac{\alpha}{\beta(\varepsilon)}\right)(\varepsilon N M+1)+\varepsilon N M$ $=\theta(\varepsilon)<k^{-2}$.

Por (a), (b), (c) e (d), temos que $s^{*}$ é uma $(M, f)$-forma, $s^{*} \in Q, \operatorname{ran}\left(s^{*}\right) \subset I(z)$ e $\left|s^{*}(z)\right|<k^{-2}$.

Finalmente, temos pelo item (d) que $\left|s^{*}(t)-y^{*}(t)\right| \leq \theta(\varepsilon), \forall t \in X$ com $\|t\|=1$, logo $\left\|s^{*}-y^{*}\right\| \leq \theta(\varepsilon)$ e $\operatorname{assim}\left|s^{*}(T(z))-y^{*}(T(z))\right|=\left|\left(s^{*}-y^{*}\right)(T(z))\right| \leq \| s^{*}-$ $y^{*}\|\| T(z)\|\leq\| s^{*}-y^{*}\|\| T\|\leq \theta(\varepsilon)\| T\left\|<\frac{\delta}{2\|T\|}\right\| T \|=\frac{\delta}{2}$. Concluímos que $s^{*}(T(z))>$ $y^{*}(T(z))-\frac{\delta}{2}>\frac{\delta}{2}$.

Lema 1.5 Sejam $X$ o espaço de Gowers e Maurey, $T \in B(X)$ e $\left(y_{n}\right)_{n=1}^{\infty}$ uma seqüência contida em $X$, de $\ell_{1+}^{n}$-averages sucessivos com constante $1+\frac{\varepsilon}{4}, \varepsilon=\frac{1}{10}$ para todo $n \in \mathbb{N}$. Então $\lim _{n \rightarrow+\infty} d\left(T\left(y_{n}\right)-\left[y_{n}\right]\right)=0$, isto é, $\lim _{n \rightarrow+\infty} \inf \left\{\left\|T\left(y_{n}\right)-\alpha y_{n}\right\|\right.$ : $\alpha \in \mathbb{R}\}=0$.

Demonstração: Suponhamos que o resultado não seja válido, então existem $T \in$ $B(X), \delta>0$ e uma subseqüência de $\left(y_{n}\right)_{n=1}^{\infty}$, que continuaremos denotando por $\left(y_{n}\right)_{n=1}^{\infty}$, tais que $d\left(T\left(y_{n}\right),\left[y_{n}\right]\right)>2 \delta$ para todo $n \in \mathbb{N}$. Seja $\left(e_{j}\right)_{j=1}^{\infty}$ a base de $X$.

Aplicamos os resultados do Lema 1.3 à seqüência $\left(y_{n}\right)_{n=1}^{\infty}$; logo existem um subespaço fechado $Y$ de $X$, um operador limitado $\bar{T}: Y \rightarrow X$ tal que, se $T^{\prime}=\left.T\right|_{Y}$, então $\left\|\bar{T}-T^{\prime}\right\|<\frac{\delta}{8}$. Além disso, obtemos uma subseqüência de $\left(y_{n}\right)_{n=1}^{\infty}$, que para facilitar a notação denotaremos novamente por $\left(y_{n}\right)_{n=1}^{\infty}$, contida em $Y$ e tal que, se $I\left(y_{n}\right)$ é 
o menor intervalo que contém supp $\left(y_{n}\right)$ e supp $\left(\bar{T}\left(y_{n}\right)\right)$, então $I\left(y_{n}\right)<I\left(y_{n+1}\right)$ para todo $n \in \mathbb{N}$.

Como consequêencia do teorema de Hahn-Banach (veja Proposição 0.13), podemos tomar $S_{n}^{*} \in X^{*}$, tal que para todo $n \in \mathbb{N},\left\|S_{n}^{*}\right\|=1, S_{n}^{*}\left(\bar{T}\left(y_{n}\right)\right)=d\left(\bar{T}\left(y_{n}\right),\left[y_{n}\right]\right)$ $>\delta$ e $S_{n}^{*}\left(y_{n}\right)=0$

Consideremos os intervalos $I\left(y_{n}\right)=\left\{n_{n}, n_{n}+1, \ldots, m_{n}\right\}$ e as projeções $E_{n}$ : $X \rightarrow\left[e_{n_{n}}, e_{n_{n}+1}, \ldots, e_{m_{n}}\right]$, definidas por $E_{n}\left(\sum_{j=1}^{\infty} a_{j} e_{j}\right)=\sum_{j=n_{n}}^{m_{n}} a_{j} e_{j}$. Definimos então, os funcionais lineares $y_{n}^{*}: X \rightarrow \mathbb{R}$ pondo $y_{n}^{*}(z)=S_{n}^{*}\left(E_{n}(z)\right), \forall z \in X$.

O funcional $y_{n}^{*}$ é limitado, pois $S_{n}^{*}$ é limitado e $\left\|E_{n}(z)\right\| \leq\|z\|$ para todo $n \in \mathbb{N}$, já que $X$ satisfaz uma $f$-estimativa inferior, além disso vale também $y_{n}^{*}\left(y_{n}\right)=S_{n}^{*}\left(E_{n}\left(y_{n}\right)\right)=S_{n}^{*}\left(y_{n}\right)=0$ e $y_{n}^{*}\left(\bar{T}\left(y_{n}\right)\right)=S_{n}^{*}\left(E_{n}\left(\bar{T}\left(y_{n}\right)\right)=S_{n}^{*}\left(\bar{T}\left(y_{n}\right)\right)>\delta\right.$. Temos também que supp $\left(y_{n}^{*}\right) \subset I\left(y_{n}\right), \operatorname{logo} \operatorname{ran}\left(y_{n}^{*}\right) \subset I\left(y_{n}\right)$. Para justificar esta última afirmação, observemos que se $z \in X, z=\sum_{j=1}^{\infty} a_{j} e_{j}$ então, $y_{n}^{*}(z)=$ $S_{n}^{*}\left(E_{n}\left(\sum_{j=1}^{\infty} a_{j} e_{j}\right)\right)=S_{n}^{*}\left(\sum_{j=n_{n}}^{m_{n}} a_{j} e_{j}\right)=\sum_{j=n_{n}}^{m_{n}} a_{j} S_{n}^{*}\left(e_{j}\right)$ e considerando $S_{n}^{*}\left(e_{j}\right)=b_{j}$, $n_{n} \leq j \leq m_{n}$, teremos $y_{n}^{*}(z)=\sum_{j=n_{n}}^{m_{n}} a_{j} b_{j}$, isto é, $\operatorname{supp}\left(y_{n}^{*}\right) \subset\left\{n_{n}, n_{n}+1, \ldots, m_{n}\right\}=$ $I\left(y_{n}\right)$.

Mostremos agora que dados $p \in L$ e $m \in \mathbb{N}$, é sempre possível tomar um vetor RIS normalizado $z \in Y$ de comprimento $p$ e constante $1+\frac{\varepsilon}{4}$ e uma $(p, f)$-forma $y^{*}$ tais que $m<I(z), \operatorname{ran}\left(y^{*}\right) \subset I(z), y^{*}(z)=0$ e $y^{*}(\bar{T}(z))>\frac{\delta}{2}$.

De fato, dados $p \in L$ e $m \in \mathbb{N}$, podemos escolher uma subseqüência finita $y_{n_{1}}, y_{n_{2}}, \ldots, y_{n_{p}}\left(n_{1}>n_{2}>\cdots>n_{p}\right)$ da seqüência $\left(y_{n}\right)_{n=1}^{\infty}$, de tal forma que $n_{1} \geq 2(1+\varepsilon) M_{f}\left(\frac{p}{\varepsilon^{\prime}}\right) / \varepsilon^{\prime} f^{\prime}(1)$ com min $\operatorname{supp}\left(y_{n_{1}}\right)>m$ e $\frac{\varepsilon^{\prime}}{2} f\left(n_{i}\right)^{1 / 2} \geq\left|\operatorname{ran}\left(y_{n_{i}-1}\right)\right|$ para cada $i=2, \ldots, p$. Logo $y_{n_{1}}, \ldots, y_{n_{p}}$ formam uma RIS de comprimento $p \in L$ e 
constante $1+\frac{\varepsilon}{4}$ tal que $m<I\left(y_{n_{1}}\right)$. Escolhemos então $z=\left(y_{n_{1}}+\cdots+y_{n_{p}}\right) / \| y_{n_{1}}+$ $\cdots+y_{n_{p}} \|$, que é um vetor RIS normalizado, pertencente a $Y$ de comprimento $p$ dado, constante $1+\frac{\varepsilon}{4}$ e tal que $m<I(z)$.

Além disso, como $p \in\left[\log p, e^{p}\right]$, temos pelo Lema 0.48 que $\left\|\sum_{i=1}^{p} y_{n_{i}}\right\| \leq \frac{2 p}{f(p)}$.

Seja agora $y^{*}=\left(y_{n_{1}}^{*}+y_{n_{2}}^{*}+\cdots+y_{n_{p}}^{*}\right) f(p)^{-1} \in X^{*}$, temos $y^{*}\left(\sum_{i=1}^{p} \bar{T}\left(y_{n_{i}}\right)\right)=$ $\left(\sum_{i=1}^{p} f(p)^{-1} y_{n_{i}}^{*}\right)\left(\sum_{i=1}^{p} \bar{T}\left(y_{n_{i}}\right)\right)=\sum_{i=1}^{p} f(p)^{-1} y_{n_{i}}^{*}\left(\bar{T}\left(y_{n_{i}}\right)\right)$, pois $\operatorname{ran}\left(y_{n_{i}}^{*}\right) \subset I\left(y_{n_{i}}\right) . \operatorname{Logo}$ $y^{*}\left(\sum_{i=1}^{p} \bar{T}\left(y_{n_{i}}\right)\right) \geq \frac{\delta p}{f(p)}$.

Temos também que $y^{*}\left(\sum_{i=1}^{p} y_{n_{i}}\right)=\sum_{i=1}^{p} f(p)^{-1} y_{n_{i}}^{*}\left(y_{n_{i}}\right)=0$. Agora, $y^{*}(\bar{T}(z))=$ $\left(\left\|\sum_{i=1}^{p} y_{n_{i}}\right\|\right)^{-1} y^{*}\left(\sum_{i=1}^{p} \bar{T}\left(y_{n_{i}}\right)\right) \geq \frac{f(p)}{2 p} \frac{\delta p}{f(p)}=\frac{\delta}{2}$ e $y^{*}(z)=\left(\left\|\sum_{i=1}^{p} y_{n_{i}}\right\|\right)^{-1} y^{*}\left(\sum_{i=1}^{p} y_{n_{i}}\right)=$ 0. Obtemos também $\operatorname{ran}\left(y^{*}\right) \subset I(z)$, pois $\operatorname{ran}\left(y_{n_{i}}^{*}\right) \subset I\left(y_{n_{i}}\right), i=1,2, \ldots, p$.

Falta mostrar que $y^{*}$ é uma $(p, f)$-forma. Pela Definição 0.38 , provemos que valem as propriedades a seguir:

(a) $f(p)^{-1} y_{n_{i}}^{*}<f(p)^{-1} y_{n_{i+1}}^{*}$, para $i=1,2, \ldots, p-1$.

(b) Seja $z \in X$ com $\|z\| \leq 1 \operatorname{logo},\left|y_{n_{i}}^{*}(z)\right|=\left|S_{n_{i}}^{*}\left(E_{n_{i}}(z)\right)\right| \leq\left\|S_{n_{i}}^{*}\right\|\left\|E_{n_{i}}(z)\right\|=$ $\left\|E_{n_{i}}(z)\right\| \leq\|z\| \leq 1, \operatorname{assim}\left\|f(p)^{-1} y_{n_{i}}^{*}\right\| \leq f(p)^{-1}$ para $i=1,2, \ldots, p$.

(c) Seja $z \in X \operatorname{com}\|z\| \leq 1$, então $\left|y^{*}(z)\right|=\left|f(p)^{-1} \sum_{i=1}^{p} y_{n_{i}}^{*}(z)\right| \leq f(p)^{-1} \sum_{i=1}^{p}\left|y_{n_{i}}^{*}(z)\right|$ $\leq f(p)^{-1} \sum_{i=1}^{p}\left\|E_{n_{i}}(z)\right\| \leq\|\xi\|$, pois $X$ satisfaz uma $f$-estimativa inferior.

Concluímos então, que $\left\|y^{*}\right\| \leq 1$.

Baseados neste resultado, faremos a seguir a seguinte construção. Dado $k \in K$, seja $z_{1} \in Y, z_{1}=\left(y_{n_{1}}+\cdots+y_{n_{M_{1}}}\right) /\left\|y_{n_{1}}+\cdots+y_{n_{M_{1}}}\right\|$ um vetor RIS normalizado 
de comprimento $M_{1}=j_{2 k} \in L$ e constante $\left(1+\frac{\varepsilon}{4}\right)$, onde pelo Lema $0.40, M_{1}^{\varepsilon / 4}=$ $N_{1} \geq 4 M f\left(\frac{k}{\varepsilon}\right) / \varepsilon f^{\prime}(1)$ e seja $y_{1}^{*}=\left(y_{n_{1}}^{*}+\cdots+y_{n_{M_{1}}}^{*}\right) f\left(M_{1}\right)^{-1}$ a $\left(M_{1}, f\right)$-forma tal que $\operatorname{ran}\left(y_{n_{i}}^{*}\right) \subset I\left(y_{n_{i}}\right), 1 \leq i \leq M_{1}, y_{1}^{*}\left(z_{1}\right)=0$ e $y_{1}^{*}\left(\bar{T}\left(z_{1}\right)\right)>\frac{\delta}{2}$.

Pelo Lema 1.4, existe uma $\left(M_{1}, f\right)$-forma $s_{1}^{*} \in Q \operatorname{com}\left|s_{1}^{*}\left(z_{1}\right)\right| \leq k^{-2}$ e $s_{1}^{*}\left(\bar{T}\left(y_{1}\right)\right)>$ $\frac{\delta}{4}$, podemos assumir também que ran $\left(s_{1}^{*}\right) \subset I\left(z_{1}\right)$.

Seja agora $p=M_{2}=\sigma\left(s_{1}^{*}\right)$ (veja a Definição 0.41). Tomemos um vetor RIS normalizado $z_{2} \in Y$ de comprimento $M_{2}$ e constante $1+\frac{\varepsilon}{4}$, tal que $m=\operatorname{máx\operatorname {supp}}\left(y_{n_{M_{1}}}\right)$ $<I\left(z_{2}\right)$ (a construção de $z_{2}$ é análoga à feita para $z_{1}$ ).

Como no caso anterior, podemos determinar pelo Lema 1.4 uma $\left(M_{2}, f\right)$-forma $s_{2}^{*} \in Q \operatorname{com}\left|s_{2}^{*}\left(z_{2}\right)\right| \leq k^{-2}, s_{2}^{*}\left(\bar{T}\left(z_{2}\right)\right)>\frac{\delta}{4}$ e $\operatorname{ran}\left(s_{2}^{*}\right) \subset I\left(z_{2}\right)$.

Continuando esta construção, dado $k \in K$, obtemos um par de seqüências $z_{2}, z_{2}, \ldots, z_{k}$ e $s_{1}^{*}, s_{2}^{*}, \ldots, s_{k}^{*}$, com várias propriedades que necessitaremos.

(1) $I\left(z_{i}\right)<I\left(z_{i+1}\right)$ para todo $i, 1 \leq i \leq k-1$.

(2) $\left\|z_{i}\right\|=1,\left\|s_{i}^{*}\right\| \leq 1$ e $\operatorname{ran}\left(s_{i}^{*}\right) \subset I\left(z_{i}\right)$, para todo $i, 1 \leq i \leq k$.

(3) $\left|s_{i}^{*}\left(z_{i}\right)\right| \leq k^{-2}$ e $s_{i}^{*}\left(\bar{T}\left(z_{i}\right)\right)>\frac{\delta}{4}$ para todo $i, 1 \leq i \leq k$.

(4) A seqüência $s_{1}^{*}, s_{2}^{*}, \ldots, s_{k}^{*}$ foi construída de forma a ser uma seqüência especial de comprimento $k$, pois $s_{i}^{*}<s_{i+1}^{*}, s_{1}^{*} \in Q, s_{i}^{*} \in A_{j_{2 k}}^{*}(X)$ e $s_{i+1}^{*} \in A_{\sigma\left(s_{1}^{*}, \ldots, s_{i}^{*}\right)}^{*}(X)$, para todo $i, 1 \leq i \leq k-1$ (veja a Definição 0.43 ).

Vamos determinar uma limitação superior para $\left\|\sum_{i=1}^{k} z_{i}\right\|$. Para isso usaremos o Lema 0.49 , porém antes é necessário que $\left|\left(\sum_{i=1}^{k} s_{i}^{*}\right)\left(\sum_{i=1}^{k} E\left(z_{i}\right)\right)\right| \leq 2$ para todo intervalo $E$. 
Desenvolvendo o módulo acima temos: $\left|\left(\sum_{i=1}^{k} s_{i}^{*}\right)\left(\sum_{i=1}^{k} E\left(z_{i}\right)\right)\right|=\left|\sum_{i=1}^{k} \sum_{j=1}^{k} s_{i}^{*}\left(E\left(z_{j}\right)\right)\right|$ $=\left|\sum_{i=1}^{k} s_{i}^{*}\left(E\left(z_{i}\right)\right)\right|$, pois $\operatorname{ran}\left(s_{i}^{*}\right) \subset I\left(z_{i}\right)$ e $I\left(z_{i}\right)<I\left(z_{i+1}\right)$ acarretam $s_{i}^{*}\left(E\left(z_{j}\right)\right)=0$ para $i \neq j$.

Podemos verificar, utilizando a demonstração do Lema 1.4, item (d), que para $s_{i}^{*}\left(E\left(z_{i}\right)\right)$ vale também $\left|s_{i}^{*}\left(E\left(z_{i}\right)\right)\right| \leq k^{-2}$, seja qual for o intervalo $E$ e $i=1, \ldots, k$.

Assim, $\left|\left(\sum_{i=1}^{k} s_{i}^{*}\right)\left(\sum_{i=1}^{k} E\left(z_{i}\right)\right)\right| \leq \sum_{i=1}^{k}\left|s_{i}^{*}\left(E\left(z_{i}\right)\right)\right| \leq k k^{-2}<2$

Utilizando o Lema 0.49, teremos $\left\|\sum_{i=1}^{k} z_{i}\right\| \leq(1+2 \varepsilon) k f(k)^{-1}<2 k f(k)^{-1}$.

Pela definição de norma (veja Definição 0.45),

$$
\left\|\sum_{i=1}^{k} \bar{T}\left(z_{i}\right)\right\| \geq \sup \left\{\left|\bar{g}\left(E\left(\sum_{i=1}^{k} \bar{T}\left(z_{i}\right)\right)\right)\right|: k \in K, \bar{g} \in B_{k}^{*}(X), E \subset \mathbb{N}\right\} .
$$

Considerando $E=\operatorname{ran}\left(\sum_{i=1}^{k} \bar{T}\left(z_{i}\right)\right)$, temos $\left\|\sum_{i=1}^{k} \bar{T}\left(z_{i}\right)\right\| \geq\left|\bar{g}\left(\sum_{i=1}^{k} \bar{T}\left(z_{i}\right)\right)\right|=$ $\left|f(k)^{-\frac{1}{2}} \sum_{j=1}^{k} g_{j}\left(\sum_{i=1}^{k} \bar{T}\left(z_{i}\right)\right)\right| \geq f(k)^{-\frac{1}{2}} \sum_{j=1}^{k} g_{j}\left(\sum_{i=1}^{k} \bar{T}\left(z_{i}\right)\right)$, onde $\left(g_{j}\right)_{j=1}^{k}$ é uma seqüência especial de comprimento $k \in K$ e $\bar{g}=f(k)^{-\frac{1}{2}} \sum_{j=1}^{k} g_{j}$ um funcional especial (veja a Definição 0.44).

Consideremos, em particular, $g_{j}=s_{j}^{*}$ para qualquer $j, 1 \leq j \leq k$. Logo, temos $\left\|\sum_{i=1}^{k} \bar{T}\left(z_{i}\right)\right\| \geq f(k)^{-\frac{1}{2}} \sum_{i=1}^{k} s_{j}^{*}\left(\sum_{i=1}^{k} \bar{T}\left(z_{i}\right)\right)=f(k)^{-\frac{1}{2}} \sum_{j=1}^{k} \sum_{i=1}^{k} s_{j}^{*}\left(\bar{T}\left(z_{i}\right)\right)$.

Como ran $\left(s_{i}^{*}\right) \subset I\left(z_{i}\right)$ e $I\left(z_{i}\right)<I\left(z_{i+1}\right)$ teremos também $s_{j}^{*}\left(\bar{T}\left(z_{i}\right)\right)=0$ se $i \neq j$. Portanto, $\left\|\sum_{i=1}^{k} \bar{T}\left(z_{i}\right)\right\| \geq f(k)^{-\frac{1}{2}} \sum_{i=1}^{k} s_{i}^{*}\left(\bar{T}\left(z_{i}\right)\right) \geq f(k)^{-\frac{1}{2}} k \frac{\delta}{4}$.

Concluímos que dado $k \in K$, é possível construir uma seqüência $z_{1}, \ldots, z_{k}$ de vetores de $Y$, tais que $\left\|\sum_{i=1}^{k} z_{i}\right\| \leq 2 k f(k)^{-1}$ e $\left\|\sum_{i=1}^{k} \bar{T}\left(z_{i}\right)\right\|=\left\|\bar{T}\left(\sum_{i=1}^{k} z_{i}\right)\right\|>k f(k)^{-\frac{1}{2}} \frac{\delta}{4}$, isto é, $\|\bar{T}\| \geq \frac{\delta f(k)^{\frac{1}{2}}}{8}$. Como $\left\|\bar{T}-T^{\prime}\right\|<\frac{\delta}{8}$, teremos $\left\|T^{\prime}\right\| \geq\|\bar{T}\|-\frac{\delta}{8} \geq \frac{\delta}{8}\left(f(k)^{\frac{1}{2}}-1\right)$, 
o que contraria a hipótese de que $T$ é um operador limitado.

Lema 1.6 Sejam $X$ o espaço de Gowers e Maurey, $T \in B(X)$ e $\left(y_{n}\right)_{n=1}^{\infty}$ uma seqüência contida em $X$ de $\ell_{1+}^{n}$-averages sucessivos com constante $1+\frac{\varepsilon}{4}, \varepsilon=\frac{1}{10}$ para todo $n \in \mathbb{N}$. Então existe $\lambda \in \mathbb{R}$, tal que $\lim _{n \rightarrow+\infty} T\left(y_{n}\right)-\lambda y_{n}=0$.

Demonstração: Se $T=0$, então tomemos $\lambda=0$, $\operatorname{logo} \lim _{n \rightarrow+\infty} T\left(y_{n}\right)-\lambda y_{n}=0$. Suponhamos $T \neq 0$. Mostremos inicialmente que, sob a hipótese do lema, é possível obter uma subseqüência $\left(y_{n_{k}}\right)_{k=1}^{\infty}$ de $\left(y_{n}\right)_{n=1}^{\infty}$ e $\lambda \in \mathbb{R}$ tais que

$$
\lim _{k \rightarrow+\infty}\left(y_{n_{k}}\right)-\lambda y_{n_{k}}=0
$$

De fato, pelo Lema 1.5 e utilizando a hipótese, obtemos $\lim _{n \rightarrow+\infty} d\left(T\left(y_{n}\right),\left[y_{n}\right]\right)=0$, o que significa, $\lim _{n \rightarrow+\infty} \inf \left\{\left\|T\left(y_{n}\right)-\alpha y_{n}\right\|: \alpha \in \mathbb{R}\right\}=0$. Podemos então determinar uma subseqüência $\left(y_{n_{i}}\right)_{i=1}^{\infty}$, tal que para todo $i \in \mathbb{N}$, inf $\left\{\left\|T\left(y_{n_{i}}\right)-\alpha y_{n_{i}}\right\|: \alpha \in \mathbb{R}\right\}<\frac{1}{i}$. Logo, existe uma seqüência $\left(\alpha_{i}\right)_{i=1}^{\infty} \in \mathbb{R}$ tal que $\left\|T\left(y_{n_{i}}\right)-\alpha_{i} y_{n_{i}}\right\|<\frac{1}{i}$, isto é, $\lim _{i \rightarrow+\infty} T\left(y_{n_{i}}\right)-\alpha_{i} y_{n_{i}}=0$.

Além disso, $\left\|\alpha_{i} y_{n_{i}}\right\|=\left|\alpha_{i}\right|=\left\|T\left(y_{n_{i}}\right)-\alpha_{i} y_{n_{i}}+T\left(y_{n_{i}}\right)\right\| \leq\left\|T\left(y_{n_{i}}\right)-\alpha_{i} y_{n_{i}}\right\|+$ $\left\|T\left(y_{n_{i}}\right)\right\| \leq C+\|T\|, C>0$, pois a seqüência $T\left(y_{n_{i}}\right)-\alpha_{i} y_{n_{i}}$ é limitada. Segue que $\left(\alpha_{i}\right)_{i=1}^{\infty}$ é limitada, logo existem uma subseqüência $\left(\alpha_{i_{k}}\right)_{k=1}^{\infty}$ e $\lambda \in \mathbb{R}$ tais que $\lim _{k \rightarrow+\infty} \alpha_{i_{k}}=\lambda$.

Obtemos então, uma subseqüência $\left(y_{n_{i_{k}}}\right)_{k=1}^{\infty}$ de $\left(y_{n}\right)_{n=1}^{\infty}$ e $\lambda \in \mathbb{R}$ tais que $\lim _{k \rightarrow+\infty} T\left(y_{n_{i_{k}}}\right)-\alpha_{i_{k}} y_{n_{i_{k}}}=0 \mathrm{e} \lim _{k \rightarrow+\infty}\left(\alpha_{i_{k}}-\lambda\right) y_{n_{i_{k}}}=0$. Logo $\lim _{k \rightarrow+\infty} T\left(y_{n_{i_{k}}}\right)-\lambda y_{n_{i_{k}}}=$ $\lim _{k \rightarrow+\infty} T\left(y_{n_{i_{k}}}\right)-\alpha_{i_{k}} y_{n_{i_{k}}}+\left(\alpha_{i_{k}}-\lambda\right) y_{n_{i_{k}}}=0$.

Suponhamos agora, que para todo $\lambda \in \mathbb{R}$ não vale $\lim _{n \rightarrow+\infty} T\left(y_{n}\right)-\lambda y_{n}=0$.

Afirmamos que neste caso, existem $c, d \in \mathbb{R}^{*}$ e subseqüências de $\left(y_{n}\right)_{n=1}^{\infty}$ que, 
para facilitar a notação, escreveremos $\left(u_{n}\right)_{n=1}^{\infty}$ e $\left(v_{n}\right)_{n=1}^{\infty}$, tais que:

$$
\lim _{n \rightarrow+\infty} T\left(u_{n}\right)-c u_{n}=0 \mathrm{e}-\lim _{n \rightarrow \infty}(T-c I)\left(v_{n}\right)-d v_{n}=0
$$

Pelo resultado (1.1) obtido anterioremente é possível encontrar $\alpha \in \mathbb{R}$ e $\left(y_{n_{k}}\right)_{k=1}^{\infty}$ uma subseqüência de $\left(y_{n}\right)_{n=1}^{\infty}$, tais que

$$
\lim _{k \rightarrow+\infty} T\left(y_{n_{k}}\right)-\alpha y_{n_{k}}=0
$$

Como não vale $\lim _{n \rightarrow+\infty} T\left(y_{n}\right)-\alpha y_{n}=0$, então existe uma outra subseqüência $\left(y_{n_{l}}\right)_{l=1}^{\infty}$ de $\left(y_{n}\right)_{n=1}^{\infty}$ e $\varepsilon_{1}>0$ tais que $\left\|T\left(y_{n_{l}}\right)-\alpha y_{n_{l}}\right\|>\varepsilon_{1}, \forall l \in \mathbb{N}$.

Aplicando o resultado (1.1) para $(T-\alpha I) \in B(X)$ e a seqüência $\left(y_{n_{l}}\right)_{l=1}^{\infty}$ de $\ell_{1^{+}}^{n_{l}}$-averages sucessivos, existe $\beta \in \mathbb{R}$ e uma subseqüência $\left(y_{n_{l_{i}}}\right)_{i=1}^{\infty}$ de $\left(y_{n_{l}}\right)_{l=1}^{\infty}$ e conseqüentemente de $\left(y_{n}\right)_{n=1}^{\infty}$, tais que

$$
\left\|T\left(y_{n_{l_{i}}}\right)-\alpha\left(y_{n_{l_{i}}}\right)\right\|>\varepsilon_{1}, \forall i \in \mathbb{N}
$$

e

$$
\lim _{i \rightarrow+\infty}(T-\alpha I)\left(y_{n_{l_{i}}}\right)-\beta y_{n_{l_{i}}}=0 .
$$

Notemos que $\beta \neq 0$, pois se $\beta=0$ teríamos por (1.5) $\lim _{i \rightarrow+\infty} T\left(y_{n_{l_{i}}}\right)-\alpha y_{n_{l_{i}}}=0$, o que contraria (1.4).

Analisemos agora $\alpha \in \mathbb{R}$. Se $\alpha \neq 0$, então por (1.3) e (1.5) a afirmação (1.2) está provada onde $c=\alpha, d=\beta, u_{n}=y_{n_{k}}$ e $v_{n}=y_{n_{l_{i}}}$.

Vamos supor então, que $\alpha=0$. Utilizando a afirmação (1.5), teremos:

$$
\lim _{i \rightarrow+\infty} T\left(y_{n l_{i}}\right)-\beta y_{n l_{i}}=0
$$

e também como não vale $\lim _{n \rightarrow+\infty} T\left(y_{n}\right)-\beta y_{n}=0$, existe uma subseqüência $\left(y_{n_{r}}\right)_{r=1}^{\infty}$ de $\left(y_{n}\right)_{n=1}^{\infty}$ e $\varepsilon_{2}>0$ tais que $\left\|T\left(y_{n_{r}}\right)-\beta\left(y_{n_{r}}\right)\right\|>\varepsilon_{2}, \quad \forall r \in \mathbb{N}$. 
Aplicando novamente o resultado (1.1) para $(T-\beta I) \in B(X)$ e à seqüência $\left(y_{n_{r}}\right)_{r=1}^{\infty}$ de $\ell_{1+}^{n_{r}}$-averages sucessivos, existe $\gamma \in \mathbb{R}$ e uma subseqüência $\left(y_{n_{r_{i}}}\right)_{i=1}^{\infty}$ de $\left(y_{n_{r}}\right)_{r=1}^{\infty}$ e conseqüentemente de $\left(y_{n}\right)_{n=1}^{\infty}$, tais que:

$$
\lim _{i \rightarrow+\infty}(T-\beta I)\left(y_{n_{r_{i}}}\right)-\gamma\left(y_{n_{r_{i}}}\right)=0
$$

Notemos que também nesse caso $\gamma \neq 0$, logo por (1.6) e (1.7) a afirmação (1.2) está provada, onde $\beta=c, \gamma=d, u_{n}=y_{n_{l_{i}}}$ e $v_{n}=y_{n_{r_{i}}}$.

Voltando ao resultado $(1.2)$, como $\lim _{n \rightarrow+\infty} T\left(u_{n}\right)-c u_{n}=0$, podemos obter uma subseqüência $\left(u_{n_{k}}\right)_{k=1}^{\infty}$ de $\left(u_{n}\right)_{n=1}^{\infty}$ tal que $\left\|T\left(u_{n_{k}}\right)-c u_{n_{k}}\right\|<\frac{1}{2^{k}} \frac{|d|}{6}, k \in \mathbb{N}$. Isto garante que $\sum_{k=1}^{\infty}\left\|T\left(u_{n_{k}}\right)-c u_{n_{k}}\right\|<\frac{|d|}{6}$.

Analogamente, podemos tomar uma subseqüência $\left(v_{n_{k}}\right)_{k=1}^{\infty}$ de $\left(v_{n}\right)_{n=1}^{\infty}$, tal que $\left\|(T-c I)\left(v_{n_{k}}\right)-d v_{n_{k}}\right\|<\frac{1}{2^{k}} \frac{|d|}{6}, \log 0 \sum_{k=1}^{\infty}\left\|(T-c I)\left(v_{n_{k}}\right)-d v_{n_{k}}\right\|<\frac{|d|}{6}$.

Sejam $L=\left[\left(u_{n_{k}}\right)_{k=1}^{\infty}\right]$ e $M=\left[\left(v_{n_{k}}\right)_{k=1}^{\infty}\right]$. Fazendo uso de argumentos idênticos aos utilizados para a seqüência $\left(y_{n}\right)_{n=1}^{\infty}$ no Lema 1.3 , concluímos que $\left(u_{n_{k}}\right)_{k=1}^{\infty}$ e $\left(v_{n_{k}}\right)_{k=1}^{\infty}$ são seqüências básicas e portanto são bases de Schauder normalizadas de $L$ e $M$, respectivamente.

Pelo Teorema $0.52, X$ é um espaço hereditariamente indecomponível, logo pelo Lema 0.53, existem $x \in L$ e $u \in M \operatorname{com}\|x\|=\|u\|=1$ e $\|x-u\|<\delta$, onde $\delta=\min \left\{\frac{|d|}{2|c|}, \frac{|d|}{6\|T\|}\right\}$.

Observemos que se $x \in L, x=\sum_{k=1}^{\infty} a_{k} u_{n_{k}} \operatorname{com}\|x\|=1$; então $\left|a_{k}\right|=\left\|a_{k} u_{n_{k}}\right\| \leq$ $\|x\|=1, \forall k \in \mathbb{N}$, já que $X$ satisfaz uma $f$-estimativa inferior.

Assim, $\|T(x)-c x\|=\left\|\sum_{k=1}^{\infty} a_{k}\left(T\left(u_{n_{k}}\right)-c u_{n_{k}}\right)\right\| \leq \sum_{k=1}^{\infty}\left|a_{k}\right|\left\|T\left(u_{n_{k}}\right)-c u_{n_{k}}\right\| \leq$
$\sum_{k=1}^{\infty}\left\|T\left(u_{n_{k}}\right)-c u_{n_{k}}\right\|<\frac{|d|}{6}$. Utilizando o mesmo raciocínio, chegamos a $\|(T-c I)(u)-$ 
$d u \|<\frac{|d|}{6}$. Concluímos que:

$$
\begin{aligned}
\|c x-(c+d) u\| & \leq\|c x-T(x)\|+\|T(x)-T(u)\|+\|T(u)-(c+d) u\| \\
& <\frac{|d|}{6}+\|T\| \frac{|d|}{6\|T\|}+\frac{|d|}{6}=\frac{|d|}{2}
\end{aligned}
$$

e também:

$$
\begin{aligned}
\|c x-(c+d) u\| & =\|-d u-(-c x+c u)\| \geq\|-d u\|-\|c(u-x)\| \\
& >|d|-|c| \frac{|d|}{2|c|}=\frac{|d|}{2}
\end{aligned}
$$

o que é uma contradição.

Lema 1.7 Se X é o espaço de Gowers e Maurey, então X contém uma seqüência normalizada $\left(x_{i}\right)_{i=1}^{\infty}$ de vetores sucessivos, tal que para todo $T \in B(X)$ existe $\lambda \in \mathbb{R}$ satisfazendo $\lim _{i \rightarrow+\infty} T\left(x_{i}\right)-\lambda x_{i}=0$.

Demonstração: Sejam $\left(e_{j}\right)_{j=1}^{\infty}$ base de $X$ e $c=1+\frac{\varepsilon}{4}, \operatorname{com} \varepsilon=\frac{1}{10}$. Pelo Lema 0.35 , existe $x_{1} \in X, x_{1}$ um $\ell_{1^{+}}^{1}$-average com constante $c$. Consideremos $n_{1} \in \mathbb{N}$, $n_{1}>\operatorname{máx} \operatorname{supp}\left(x_{1}\right)$ e $X_{2}=\left[\left(e_{j}\right)_{j=n_{1}}^{\infty}\right]$.

Como $X_{2}$ é um subespaço de bloco de $X$ existe novamente, pelo Lema 0.35 , um vetor $x_{2} \in X_{2}<X, x_{2}$ um $\ell_{1+}^{2}$-average com constante $c$.

Continuando este raciocínio, obtemos uma seqüência $\left(x_{i}\right)_{i=1}^{\infty}$ contida em $X$, tal que $x_{i} \in X_{i}=\left[\left(e_{j}\right)_{j=n_{i-1}}^{\infty}\right]\left(n_{0}=1\right)$ e $x_{i}$ é um $\ell_{1^{+}}^{i}$-average com constante $c$, para todo $i \in \mathbb{N}$.

Assim, $\left(x_{i}\right)_{i=1}^{\infty}$ é uma seqüencia normalizada de vetores sucessivos de $X$. Aplicando o Lema 1.6, dado $T \in B(X)$, existe $\lambda \in \mathbb{R}$, tal que $\lim _{i \rightarrow+\infty} T\left(x_{i}\right)-\lambda x_{i}=0$. 
Vamos agora associar, à seqüência $\left(x_{i}\right)_{i=1}^{\infty}$ do lema anterior, uma seqüência de funcionais lineares $\left(x_{i}^{*}\right)_{i=1}^{\infty}$ contida em $X^{*}$. Estes funcionais são chamados de funcionais suporte para a seqüência $\left(x_{i}\right)_{i=1}^{\infty}$.

Lema 1.8 Se $X$ é o espaço de Gowers e Maurey $e\left(x_{i}\right)_{i=1}^{\infty}$ é a seqüência de vetores contida em $X$ com as propriedades do lema anterior, então existe uma seqüencia $\left(x_{i}^{*}\right)_{i=1}^{\infty}$ contida em $X^{*}$ de funcionais lineares, tais que $\operatorname{supp}\left(x_{i}^{*}\right) \subset \operatorname{ran}\left(x_{i}\right)$. Além disso, $x_{i}^{*}\left(x_{j}\right)=\delta_{i j}$ e $\left\|x_{i}^{*}\right\|=1$, para todo $i \in \mathbb{N}$.

Demonstração: Sejam $\left(x_{i}\right)_{i=1}^{\infty}$ a seqüência do lema anterior e $R_{i}^{*}:\left[x_{i}\right] \rightarrow \mathbb{R}$ definido por $R_{i}^{*}\left(\alpha x_{i}\right)=\alpha$. O funcional $R_{i}^{*} \in X^{*}$ para todo $i \in \mathbb{N}$; pois $R_{i}^{*}\left(\alpha x_{i}+\beta x_{i}\right)=$ $R_{i}^{*}\left((\alpha+\beta) x_{i}\right)=\alpha+\beta=R_{i}^{*}\left(\alpha x_{i}\right)+R_{i}^{*}\left(\beta x_{i}\right)$ e $R_{i}^{*}\left(\gamma\left(\alpha x_{i}\right)\right)=R_{i}^{*}\left((\gamma \alpha) x_{i}\right)=\gamma \alpha=$ $\gamma R_{i}^{*}\left(\alpha x_{i}\right)$.

Temos também que $\sup \left\{\left|R_{i}^{*}\left(\alpha x_{i}\right)\right|:\left\|\alpha x_{i}\right\| \leq 1\right\}=\sup \{|\alpha|:|\alpha| \leq 1\} \leq 1$. Além disso $\left\|R_{i}^{*}\right\|=1$, pois $R_{i}^{*}\left(x_{i}\right)=1$ e $\left\|x_{i}\right\|=1$.

Consideremos $\operatorname{ran}\left(x_{i}\right)=\left\{n_{i}, n_{i}+1, \ldots, n_{i}+m_{i}\right\}$. Como conseqüência do Teorema de Hahn-Banach (veja o Corolário 0.14), existe $\overline{R_{i}^{*}}:\left[e_{n_{i}}, e_{n_{i}+1}, \ldots, e_{n_{i}+m_{i}}\right]$ $\rightarrow \mathbb{R}, \overline{R_{i}^{*}} \in X^{*}$, tal que $\left.\overline{R_{i}^{*}}\right|_{\left[x_{i}\right]}=R_{i}^{*}$ e $\left\|\overline{R_{i}^{*}}\right\|=\left\|R_{i}^{*}\right\|=1$.

Dado $x=\sum_{j=1}^{\infty} a_{j} e_{j}$, sejam $E_{i}: X \rightarrow\left[e_{n_{i}}, e_{n_{i}+1}, \ldots, e_{n_{i}+m_{i}}\right]$, as projeções dadas por $E_{i}\left(\sum_{j=1}^{\infty} a_{j} e_{j}\right)=\sum_{j=n_{i}}^{n_{i}+m_{i}} a_{j} e_{j}$.

Vamos agora definir $x_{i}^{*}: X \rightarrow \mathbb{R}$ por $x_{i}^{*}(x)=\overline{R_{i}^{*}}\left(E_{i}(x)\right), \forall x \in X$. Cada $x_{i}^{*} \in X^{*}$, pois $\overline{R_{i}^{*}} \in X^{*}$ e decorrente da desigualdade $\left\|E_{i}(x)\right\| \leq\|x\|, \forall x \in X$, temos que $E_{i}: X \rightarrow\left[e_{n_{i}}, e_{n_{i}+1}, \ldots, e_{n_{i}+m_{i}}\right]$ é uma aplicação linear limitada.

A seqüência $\left(x_{i}^{*}\right)_{i=1}^{\infty}$ assim construída, satisfaz as seguintes propriedades: 
(1) $x_{i}^{*}\left(x_{j}\right)=\delta_{i j}$. De fato, se $i=j$, então $x_{i}^{*}\left(x_{j}\right)=\overline{R_{i}^{*}}\left(E_{i}\left(x_{i}\right)\right)=\overline{R_{i}^{*}}\left(x_{i}\right)=R_{i}\left(x_{i}\right)=$ 1 e se $i \neq j$, então $x_{i}^{*}\left(x_{j}\right)=\overline{R_{i}^{*}}\left(E_{i}\left(x_{j}\right)\right)=\overline{R_{i}^{*}}(0)=0$.

(2) $\left\|x_{i}^{*}\right\|=1$, pois $\sup \left\{\left|\overline{R_{i}^{*}}\left(E_{i}(x)\right)\right|:\|x\| \leq 1\right\} \leq \sup \left\{\left\|\overline{R_{i}^{*}}\right\|\left\|E_{i}(x)\right\|:\|x\| \leq 1\right\} \leq$ 1 , mas $x_{i}^{*}\left(x_{i}\right)=1$ e $\left\|x_{i}\right\|=1, \operatorname{logo}\left\|x_{i}^{*}\right\|=1$.

(3) $\operatorname{supp}\left(x_{i}^{*}\right) \subset \operatorname{ran}\left(x_{i}\right)$

Seja $x \in X, x=\sum_{j=1}^{\infty} a_{j} e_{j}$, então $x_{i}^{*}(x)=\overline{R_{i}^{*}}\left(E_{i}\left(\sum_{j=1}^{\infty} a_{j} e_{j}\right)\right)=\overline{R_{i}^{*}}\left(\sum_{j=n_{i}}^{n_{i}+m_{i}} a_{j} e_{j}\right)$ $=\sum_{j=n_{i}}^{n_{i}+m_{i}} a_{j} \overline{R_{i}^{*}}\left(e_{j}\right)$. Considerando $\overline{R_{i}^{*}}\left(e_{j}\right)=b_{j}, n_{i} \leq j \leq n_{i}+m_{i}$, teremos $x_{i}^{*}(x)=$ $\sum_{j=n_{i}}^{n_{i}+m_{i}} a_{j} b_{j}$. Podemos então escrever, $\operatorname{supp}\left(x_{i}^{*}\right) \subset\left\{n_{i}, n_{i}+1, \ldots, n_{i}+m_{i}\right\}=$ $\operatorname{ran}\left(x_{i}\right)$.

(4) $\left(x_{i}^{*}\right)_{i=1}^{\infty}$ é uma seqüência de vetores sucessivos. Como $\operatorname{supp}\left(x_{i}^{*}\right) \subset \operatorname{ran}\left(x_{i}\right)$ e $\operatorname{ran}\left(x_{i}\right)<\operatorname{ran}\left(x_{i+1}\right)$ para todo $i \in \mathbb{N}$, então máx supp $\left(x_{i}^{*}\right)<\min \operatorname{supp}\left(x_{i+1}^{*}\right)$.

Vamos iniciar agora a construção do espaço de Banach W, espaço importante na apresentação da primeira solução para o problema de Schroeder-Bernstein.

Sejam $X$ o espaço de Gowers e Maurey, $\mathcal{W}=(X \underset{\infty}{\oplus} X)=\left\{\left(z_{1}, z_{2}\right): z_{1}, z_{2} \in X\right\}$ com $\left\|\left(z_{1}, z_{2}\right)\right\|=\operatorname{máx}\left\{\left\|z_{1}\right\|,\left\|z_{2}\right\|\right\}$ e $\left(\mathcal{W}_{j}\right)_{j=1}^{\infty}$ uma seqüência de cópias isométricas de $\mathcal{W}$.

Definição 1.9 Definimos um espaço vetorial $\mathrm{V}$ do seguinte modo: $\mathrm{V}=\left\{\left(w_{1}, w_{2}\right.\right.$, $\left.\ldots, w_{j}, \ldots\right): w_{j} \in \mathcal{W}$ e $w_{j} \neq 0$ somente para um número finito de índices $\left.j \in \mathbb{N}\right\}$.

Observação 1.10 Consideraremos cada cópia $\mathcal{W}_{j}$ um subespaço vetorial de $\mathrm{V}$, através do isomorfismo sobre a imagem, $T: \mathcal{W}_{j} \rightarrow \mathrm{V}$ definido por $T\left(w_{j}\right)=$ $\left(0,0, \ldots, w_{j}, 0, \ldots\right) \in \mathrm{V}$ 
Faremos então a seguinte identificação: $\mathcal{W}_{j}=\left\{\left(0,0, \ldots, w_{j}, 0, \ldots\right): w_{j} \in \mathcal{W}\right\}$ e o vetor $\mathrm{w}_{j}$ de $\mathrm{V}$ como o vetor $\mathrm{w}_{j}=\left(0,0, \ldots, w_{j}, 0, \ldots\right)$, onde $w_{j} \in \mathcal{W}$.

Definição 1.11 Seja $\mathrm{w} \in \mathrm{V}$, definimos $\operatorname{supp}(\mathrm{w})=\left\{j \in \mathbb{N}: w_{j} \neq 0\right\}$ e escreveremos $\mathrm{w}<\mathrm{u}$, se máx $\operatorname{supp}(\mathrm{w})<\min \operatorname{supp}(\mathrm{u})$.

Fixemos agora uma seqüência normalizada de vetores $\left(x_{i}\right)_{i=1}^{\infty}$ de $X$, satisfazendo a propriedade do Lema 1.7 e também a seqüência $\left(x_{i}^{*}\right)_{i=1}^{\infty}$ de funcionais suporte para $\left(x_{i}\right)_{i=1}^{\infty}$ definida no Lema 1.8.

Estas duas seqüências serão muito utilizadas no decorrer do trabalho. Inicialmente vamos definir funcionais lineares limitados que serão utilizados na definição de uma norma que daremos ao espaço $\mathbf{V}$; antes porém faremos a seguinte observação.

Observação 1.12 Temos, $\mathcal{W}^{*}=\left(X \bigoplus_{\infty} X\right)^{*} \sim\left(X^{*} \oplus_{\infty} X^{*}\right)$, pelo isomorfismo $T$ : $\left(X^{*} \oplus_{\infty} X^{*}\right) \rightarrow\left(X \bigoplus_{\infty} X\right)^{*}$ definido por $T\left(z_{1}^{*}, z_{2}^{*}\right)\left(z_{1}, z_{2}\right)=z_{1}^{*}\left(z_{1}\right)+z_{2}^{*}\left(z_{2}\right)$.

Assim, identificaremos cada funcional linear limitado $w^{*} \in \mathcal{W}^{*}$ com um funcional linear limitado $\left(z_{1}^{*}, z_{2}^{*}\right) \in\left(X^{*} \oplus X^{*}\right)$ tal que $w^{*}\left(z_{1}, z_{2}\right)=z_{1}^{*}\left(z_{1}\right)+z_{2}^{*}\left(z_{2}\right),\left(z_{1}, z_{2}\right) \in$ $(X \underset{\infty}{\oplus} X), \operatorname{com}\left\|\left(z_{1}^{*}, z_{2}^{*}\right)\right\|=\operatorname{máx}\left\{\left\|z_{1}^{*}\right\|,\left\|z_{2}^{*}\right\|\right\}$.

Definição 1.13 Para cada $i, j \in \mathbb{N}$, definimos os funcionais lineares limitados $u_{i j}^{*}, v_{i j}^{*}$ e $w_{i j}^{*}$ em $\mathcal{W}_{j}^{*}$ como cópias dos funcionais $\left(x_{i}^{*},-x_{i}^{*}\right),\left(x_{i}^{*}, 0\right)$ e $\left(0, x_{i}^{*}\right)$, respectivamente.

Observação 1.14 Os funcionais $u_{i j}^{*}$, $v_{i j}^{*}$ e $w_{i j}^{*}$ pertencem também a $V^{*}$. Por exemplo, fixado $i \in \mathbb{N}, u_{i j}^{*}: \mathbf{V} \rightarrow \mathbb{R}$ é definido por $u_{i j}^{*}(\mathbf{w})=u_{i j}^{*}\left(w_{1}, w_{2}, \ldots, w_{j}, \ldots\right)=$ $u_{i j}^{*}\left(0,0, \ldots, w_{j}, 0, \ldots\right)=u_{i j}^{*}\left(w_{j}\right)=\left(x_{i}^{*},-x_{i}^{*}\right)\left(z_{1 j}, z_{2 j}\right)=x_{i}^{*}\left(z_{1 j}\right)+\left(-x_{i}^{*}\right)\left(z_{2 j}\right)$, onde $\mathrm{w} \in \mathrm{V}$ e $\left(z_{1 j}, z_{2 j}\right)=w_{j}$. 
Antes de definirmos uma conveniente norma em $\mathrm{V}$, será necessário que façamos uma partição de $\mathbb{N}$ em cinco subconjuntos infinitos que denominaremos $N_{0}, N_{1}, N_{2}$, $N_{3}$ e $N_{4}$.

Dada uma seqüência $\mathbf{w}=\left(w_{1}, w_{2}, \ldots, w_{j}, \ldots\right) \in \mathbf{V}$, definimos $\|\cdot\|_{V}: \mathbf{V} \rightarrow \mathbb{R}_{+}$ por:

$$
\begin{aligned}
\|\mathrm{w}\|_{V}= & \sup \left\{\left\|w_{j}\right\|: j \in \mathbb{N}\right\} \vee \sup \left\{\left(\sum_{j=1}^{\infty}\left|u_{i j}^{*}(\mathrm{w})\right|^{2}\right)^{\frac{1}{2}}: i \in N_{1}\right\} \\
& \vee \sup \left\{\left(\sum_{j=1}^{\infty}\left|v_{i j}^{*}(\mathrm{w})\right|^{2}\right)^{\frac{1}{2}}: i \in N_{2}\right\} \vee \sup \left\{\left(\sum_{j=1}^{\infty}\left|w_{i j}^{*}(\mathrm{w})\right|^{2}\right)^{\frac{1}{2}}: i \in N_{3}\right\} \\
& \vee \sup \left\{\left(\sum_{j=1}^{\infty}\left|v_{i j}^{*}(\mathrm{w})\right|^{4}+\left.w_{i j}^{*}(\mathrm{w})\right|^{4}\right)^{\frac{1}{4}}: i \in N_{4}\right\}
\end{aligned}
$$

Observemos inicialmente que $\|\cdot\|_{V}: V \rightarrow \mathbb{R}_{+}$está bem definida; pois $\sup \left\{\left\|w_{j}\right\|:\right.$ $j \in \mathbb{N} \|\}<\infty$, já que $w_{j} \neq 0$ somente para um número finito de índices $j \in \mathbb{N}$. Temos também sup $\left\{\left(\sum_{j=1}^{\infty}\left|u_{i j}^{*}(\mathrm{w})\right|^{2}\right)^{\frac{1}{2}}: i \in N_{1}\right\}<\infty$.

De fato, sejam $j \in \mathbb{N}$ e $\mathrm{w} \in \mathrm{V}$. Fixado $i \in N_{1}$, temos $\left|u_{i j}^{*}(\mathbf{w})\right|=\mid u_{i j}^{*}\left(w_{1}, w_{2}, \ldots\right.$, $\left.w_{j}, \ldots\right)|=| u_{i j}^{*}\left(w_{j}\right) \mid \leq\left\|u_{i j}^{*}\right\|\left\|w_{j}\right\|=\operatorname{máx}\left\{\left\|x_{i}^{*}\right\|,\left\|-x_{i}^{*}\right\|\right\}\left\|w_{j}\right\|=\left\|w_{j}\right\|$. Sejam $P=$ $\operatorname{supp}(\mathrm{w}), S=\sup \left\{\left\|w_{j}\right\|: j \in P\right\}$ e $|P|$ a cardinalidade do conjunto $P$. Então, $\left(\sum_{j=1}^{\infty}\left|u_{i j}^{*}(\mathrm{w})\right|^{2}\right)^{\frac{1}{2}}=\left(\sum_{j \in P}\left|u_{i j}^{*}(\mathrm{w})\right|^{2}\right)^{\frac{1}{2}} \leq\left(\sum_{j \in P}\left\|w_{j}\right\|^{2}\right)^{\frac{1}{2}} \leq\left(|P| S^{2}\right)^{\frac{1}{2}} \leq|P| S<\infty$. Assim, sup $\left\{\left(\sum_{j=1}^{\infty}\left|u_{i j}^{*}(\mathrm{w})\right|^{2}\right)^{\frac{1}{2}}: i \in N_{1}\right\} \leq|P| S<\infty$. Raciocínios análogos nos levam a:

$$
\begin{aligned}
& \sup \left\{\left(\sum_{j=1}^{\infty}\left|v_{i j}^{*}(\mathrm{w})\right|^{2}\right)^{\frac{1}{2}}: i \in N_{2}\right\} \leq|P| S<\infty, \\
& \sup \left\{\left(\sum_{j=1}^{\infty}\left|w_{i j}^{*}(\mathrm{w})\right|^{2}\right)^{\frac{1}{2}}: i \in N_{3}\right\} \leq|P| S<\infty
\end{aligned}
$$




$$
\text { e } \sup \left\{\left(\sum_{j=1}^{\infty}\left|v_{i j}^{*}(\mathrm{w})\right|^{4}+\sum_{j=1}^{\infty}\left|w_{i j}^{*}(\mathrm{w})\right|^{4}\right)^{\frac{1}{4}}: i \in N_{4}\right\} \leq 2|P| S<\infty
$$

Mostremos agora que $\|\mathrm{w}\|_{V}$ é norma em $\mathrm{V}$.

(1) Se $\|\mathrm{w}\|_{V}=0$ então, $\sup \left\{\left\|w_{j}\right\|: j \in \mathbb{N}\right\}=0$ logo, $\left\|w_{j}\right\|=0$ e portanto $\mathbf{w}=0$.

Se $\mathrm{w}=0$ então, $\left\|w_{j}\right\|=0 \operatorname{logo} \sup \left\{\left\|w_{j}\right\|: j \in \mathbb{N}\right\}=0$ e $u_{i j}^{*}(\mathrm{w})=0, v_{i j}^{*}(\mathrm{w})=0$, $w_{i j}^{*}(\mathrm{w})=0$, para todo $j \in \mathbb{N}$ e $i \in \mathbb{N}$ segue que $\|\mathrm{w}\|_{V}=0$.

(2) $\|\mathrm{w}\|_{V} \geq \sup \left\{\left\|w_{j}\right\|: j \in \mathbb{N}\right\} \geq 0$

(3) $\|\lambda \mathrm{w}\|_{V}=|\lambda|\|\mathrm{w}\|_{V}, \forall \lambda \in \mathbb{R}$.

Inicialmente temos que $\sup \left\{\left\|\lambda w_{j}\right\|: j \in \mathbb{N}\right\}=\sup \left\{|\lambda|\left\|w_{j}\right\|: j \in \mathbb{N}\right\}=$ $|\lambda| \sup \left\{\left\|w_{j}\right\|: j \in \mathbb{N}\right\}$. Sejam $\mathrm{w} \in \mathrm{V}$ e $j \in \mathbb{N}$, fixado $i \in N_{1}$, temos $\left|u_{i j}^{*}(\lambda \mathrm{w})\right|^{2}=$ $\left|\lambda u_{i j}^{*}(\mathrm{w})\right|^{2}=|\lambda|^{2}\left|u_{i j}^{*}(\mathrm{w})\right|^{2}, \log \mathrm{o}\left(\sum_{j=1}^{\infty}\left|u_{i j}^{*}(\lambda \mathrm{w})\right|^{2}\right)^{\frac{1}{2}}=\left(|\lambda|^{2} \sum_{j=1}^{\infty}\left|u_{i j}^{*}(\mathrm{w})\right|^{2}\right)^{\frac{1}{2}}=$ $|\lambda|\left(\sum_{j=1}^{\infty}\left|u_{i j}^{*}(\mathrm{w})\right|^{2}\right)^{\frac{1}{2}}$.

Assim, sup $\left\{\left(\sum_{j=1}^{\infty}\left|u_{i j}^{*}(\lambda \mathrm{w})\right|^{2}\right)^{\frac{1}{2}}: i \in N_{1}\right\}=\sup \left\{|\lambda|\left(\sum_{j=1}^{\infty}\left|u_{i j}^{*}(\mathrm{w})\right|^{2}\right)^{\frac{1}{2}}: i \in N_{1}\right\}$ $=|\lambda| \sup \left\{\left(\sum_{j=1}^{\infty}\left|u_{i j}^{*}(\mathrm{w})\right|^{2}\right)^{\frac{1}{2}}: i \in N_{1}\right\}$. De forma análoga obteremos $\sup \left\{\left(\sum_{j=1}^{\infty}\left|v_{i j}^{*}(\lambda \mathrm{w})\right|^{2}\right)^{\frac{1}{2}}: i \in N_{2}\right\}=|\lambda| \sup \left\{\left(\sum_{j=1}^{\infty}\left|v_{i j}^{*}(\mathrm{w})\right|^{2}\right)^{\frac{1}{2}}: i \in N_{2}\right\}$, $\sup \left\{\left(\sum_{j=1}^{\infty}\left|w_{i j}^{*}(\lambda \mathrm{w})\right|^{2}\right)^{\frac{1}{2}}: i \in N_{3}\right\}=|\lambda| \sup \left\{\left(\sum_{j=1}^{\infty}\left|w_{i j}^{*}(\mathrm{w})\right|^{2}\right)^{\frac{1}{2}}: i \in N_{3}\right\}$ $\mathrm{e} \quad \sup \left\{\left(\sum_{j=1}^{\infty}\left|v_{i j}^{*}(\lambda \mathrm{w})\right|^{4}+\left|w_{i j}^{*}(\lambda \mathrm{w})\right|^{4}\right)^{\frac{1}{4}}: i \in N_{4}\right\}$ $=|\lambda| \sup \left\{\left(\sum_{j=1}^{\infty}\left|v_{i j}^{*}(\mathrm{w})\right|^{4}+\left|w_{i j}^{*}(\mathrm{w})\right|^{4}\right)^{\frac{1}{4}}: i \in N_{4}\right\}$. 
Concluímos que:

$$
\begin{aligned}
\|\lambda \mathrm{w}\|_{V}= & |\lambda|\left(\sup \left\{\left\|w_{j}\right\|: j \in \mathbb{N}\right\} \vee \sup \left\{\left(\sum_{j=1}^{\infty}\left|u_{i j}^{*}(\mathrm{w})\right|^{2}\right)^{\frac{1}{2}}: i \in N_{1}\right\}\right. \\
& \vee \sup \left\{\left(\sum_{j=1}^{\infty}\left|v_{i j}^{*}(\mathrm{w})\right|^{2}\right)^{\frac{1}{2}}: i \in N_{2}\right\} \vee \sup \left\{\left(\sum_{j=1}^{\infty}\left|w_{i j}^{*}(\mathrm{w})\right|^{2}\right)^{\frac{1}{2}}: i \in N_{3}\right\} \\
& \left.\vee \sup \left\{\left(\sum_{j=1}^{\infty}\left|v_{i j}^{*}(\mathrm{w})\right|^{4}+\sum_{j=1}^{\infty}\left|w_{i j}^{*}(\mathrm{w})\right|^{4}\right)^{\frac{1}{4}}: i \in N_{4}\right\}\right)=|\lambda|\|\mathrm{w}\|_{V} .
\end{aligned}
$$

(4) $\|\mathrm{w}+\mathrm{u}\|_{V} \leq\|\mathrm{w}\|_{V}+\|\mathrm{u}\|_{V}, \forall \mathrm{w}, \mathrm{u} \in \mathrm{V}$.

Caso 1: $\|\mathrm{w}+\mathrm{u}\|_{V}=\sup \left\{\left\|w_{j}+u_{j}\right\|: j \in \mathbb{N}\right\}$

$$
\left\|w_{j}+u_{j}\right\| \leq\left\|w_{j}\right\|+\left\|u_{j}\right\|, \forall j \in \mathbb{N}
$$

$\log o \sup \left\{\left\|w_{j}+u_{j}\right\|: j \in \mathbb{N}\right\} \leq \sup \left\{\left\|w_{j}\right\|+\left\|u_{j}\right\|: j \in \mathbb{N}\right\}=\sup \left\{\left\|w_{j}\right\|: j \in\right.$ $\mathbb{N}\}+\sup \left\{\left\|u_{j}\right\|: j \in \mathbb{N}\right\} \leq\|\mathrm{w}\|_{V}+\|\mathbf{u}\|_{V}$.

Caso 2: $\|\mathrm{w}+\mathrm{u}\|_{V}=\sup \left\{\left(\sum_{j=1}^{\infty}\left|u_{i j}^{*}(\mathrm{w}+\mathrm{u})\right|^{2}\right)^{\frac{1}{2}}: i \in N_{1}\right\}$.

Sejam $\mathrm{w}, \mathrm{u} \in \mathrm{V}$ e $j \in \mathbb{N}$. Fixado $i \in N_{1}$, temos $\left|u_{i j}^{*}(\mathrm{w}+\mathrm{u})\right|^{2}=\mid u_{i j}^{*}(\mathrm{w})+$ $\left.u_{i j}^{*}(\mathrm{u})\right|^{2} \leq\left(\left|u_{i j}^{*}(\mathrm{w})\right|+\left|u_{i j}^{*}(\mathbf{u})\right|\right)^{2}$.

$$
\begin{aligned}
& \operatorname{Logo},\left(\sum_{j=1}^{\infty}\left|u_{i j}^{*}(\mathrm{w}+\mathrm{u})\right|^{2}\right)^{\frac{1}{2}} \leq\left(\sum_{j=1}^{\infty}\left(\left|u_{i j}^{*}(\mathbf{w})\right|+\left|u_{i j}^{*}(\mathbf{u})\right|\right)^{2}\right)^{\frac{1}{2}} \leq\left(\sum_{j=1}^{\infty}\left|u_{i j}^{*}(\mathbf{w})\right|^{2}\right)^{\frac{1}{2}}+ \\
& \left(\sum_{j=1}^{\infty}\left|u_{i j}^{*}(\mathbf{u})\right|^{2}\right)^{\frac{1}{2}} .
\end{aligned}
$$$$
\text { Assim, } \sup \left\{\left(\sum_{j=1}^{\infty}\left|u_{i j}^{*}(\mathrm{w}+\mathrm{u})\right|^{2}\right)^{\frac{1}{2}}: i \in N_{1}\right\} \leq \sup \left\{\left(\sum_{j=1}^{\infty}\left|u_{i j}^{*}(\mathrm{w})\right|^{2}\right)^{\frac{1}{2}}\right.
$$$$
\left.+\left(\sum_{j=1}^{\infty}\left|u_{i j}^{*}(\mathrm{u})\right|^{2}\right)^{\frac{1}{2}}: i \in N_{1}\right\}=\sup \left\{\left(\sum_{j=1}^{\infty}\left|u_{i j}^{*}(\mathrm{w})\right|^{2}\right)^{\frac{1}{2}}: i \in N_{1}\right\}+
$$$$
\sup \left\{\left(\sum_{i=1}^{\infty}\left|u_{i j}^{*}(\mathrm{u})\right|^{2}\right)^{\frac{1}{2}}: i \in N_{1}\right\} \leq\|\mathrm{w}\|_{V}+\|\mathrm{u}\|_{V} \text {. }
$$ 
De forma análoga obteremos $\|\mathrm{u}+\mathrm{w}\|_{V} \leq\|\mathrm{u}\|_{V}+\|\mathrm{w}\|_{V}$ para os casos:

$$
\begin{aligned}
& \|\mathrm{u}+\mathrm{w}\|_{V}=\sup \left\{\left(\sum_{j=1}^{\infty}\left|v_{i j}^{*}(\mathrm{w}+\mathrm{u})\right|^{2}\right)^{\frac{1}{2}}: i \in N_{2}\right\}, \\
& \|\mathrm{u}+\mathrm{w}\|_{V}=\sup \left\{\left(\sum_{j=1}^{\infty}\left|w_{i j}^{*}(\mathrm{w}+\mathrm{u})\right|^{2}\right)^{\frac{1}{2}}: i \in N_{3}\right\} .
\end{aligned}
$$

Finalmente, se $\|\mathrm{w}+\mathrm{u}\|=\sup \left\{\left(\sum_{j=1}^{\infty}\left|v_{i j}^{*}(\mathrm{w}+\mathrm{u})\right|^{4}+\left|w_{i j}^{*}(\mathrm{w}+\mathrm{u})\right|^{4}\right)^{\frac{1}{4}}: i \in N_{4}\right\}$, te$\operatorname{remos}\left(\sum_{j=1}^{\infty}\left|v_{i j}^{*}(\mathbf{w}+\mathbf{u})\right|^{4}+\left|w_{i j}^{*}(\mathbf{w}+\mathbf{u})\right|^{4}\right)^{\frac{1}{4}}=\left(\sum_{j=1}^{\infty}\left|v_{i j}^{*}(\mathbf{w})+v_{i j}^{*}(\mathbf{u})\right|^{4}+\mid w_{i j}^{*}(\mathbf{w})\right.$ $\left.+\left.w_{i j}^{*}(\mathrm{u})\right|^{4}\right)^{\frac{1}{4}} \leq\left(\sum_{j=1}^{\infty}\left|v_{i j}^{*}(\mathbf{w})\right|^{4}+\left|w_{i j}^{*}(\mathbf{w})\right|^{4}\right)^{\frac{1}{4}}+\left(\sum_{j=1}^{\infty}\left|v_{i j}^{*}(\mathbf{u})\right|^{4}+\left|w_{i j}^{*}(\mathbf{u})\right|^{4}\right)^{\frac{1}{4}}$

Assim, $\sup \left\{\left(\sum_{j=1}^{\infty}\left|v_{i j}^{*}(\mathbf{w}+\mathbf{u})\right|^{4}+\left|w_{i j}^{*}(\mathbf{w}+\mathbf{u})\right|^{4}\right)^{\frac{1}{4}}: i \in N_{4}\right\}$ $\leq \sup \left\{\left(\sum_{j=1}^{\infty}\left|v_{i j}^{*}(\mathrm{w})\right|^{4}+\left|w_{i j}^{*}(\mathrm{w})\right|^{4}\right)^{\frac{1}{4}}+\left(\sum_{j=1}^{\infty}\left|v_{i j}^{*}(\mathrm{u})\right|^{4}+\left|w_{i j}^{*}(\mathrm{u})\right|^{4}\right)^{\frac{1}{4}}: i \in N_{4}\right\}$ $=\sup \left\{\left(\sum_{j=1}^{\infty}\left|v_{i j}^{*}(\mathrm{w})\right|^{4}+\left|w_{i j}^{*}(\mathrm{w})\right|^{4}\right)^{\frac{1}{4}}: i \in N_{4}\right\}+\sup \left\{\left(\sum_{j=1}^{\infty}\left|v_{i j}^{*}(\mathrm{w})\right|^{4}+\left|w_{i j}^{*}(\mathrm{w})\right|^{4}\right)^{\frac{1}{4}}:\right.$ $\left.i \in N_{4}\right\}=\|\mathrm{w}\|_{V}+\|\mathrm{u}\|_{V}$.

Definição $1.15 \mathrm{O}$ completado de $\left(\mathrm{V},\|\cdot\|_{V}\right)$ é o espaço de Banach que denominaremos de W.

Observação 1.16 Cada cópia $\mathcal{W}_{j}$ de $\mathcal{W}=(X \underset{\infty}{\oplus X})$ é isomorfa ao subespaço $\mathcal{W}_{j}=$ $\left\{\left(0, \ldots, w_{j}, 0,0, \ldots\right): w_{j} \in W\right\}$ de $\mathbf{V}$ ou $\mathbf{W}$. De fato, pela definição de norma em $\mathrm{V}$ e em $\left(X \oplus_{\infty}^{\oplus} X\right)$, teremos $\left\|w_{j}\right\| \leq\left\|\left(0,0, \ldots, w_{j}, 0, \ldots\right)\right\|_{V} \leq 2\left\|w_{j}\right\|$.

Concluímos também que cada subespaço $\mathcal{W}_{j}$ de $\mathrm{W}$ é fechado.

Lema 1.17 A seqüência $\left(\mathcal{W}_{j}\right)_{j=1}^{\infty}$ de subespaços de $\mathrm{W}$ é uma decomposição de Schauder de W. 
Demonstração: Inicialmente observemos que $\mathcal{W}_{j} \neq\{0\}, \forall j \in \mathbb{N}$. Dado w $\in$ $\mathrm{V}$, com máx supp $(\mathbf{w})=p$, temos que $\mathbf{w}=\left(w_{1}, w_{2}, \ldots, w_{p}, 0,0, \ldots\right)=\sum_{j=1}^{p} \mathbf{w}_{j} \epsilon$ $\left[\bigcup_{j=1}^{\infty} \mathcal{W}_{j}\right]$. Logo, $\mathrm{V} \subset\left[\bigcup_{j=1}^{\infty} \mathcal{W}_{j}\right]$.

Como $\bigcup_{j=1}^{\infty} \mathcal{W}_{j} \subset \mathrm{W}$, então $\left[\bigcup_{j=1}^{\infty} \mathcal{W}_{j}\right] \subset \mathrm{W}$, pois $\mathrm{W}$ é fechado. Ainda, pelo fato de que $\mathrm{V} \subset\left[\bigcup_{j=1}^{\infty} \mathcal{W}_{j}\right]$, temos $[\mathrm{V}] \subset\left[\bigcup_{j=1}^{\infty} \mathcal{W}_{j}\right] ;$ mas $[\mathrm{V}]=\mathrm{W}$

Concluímos que $\mathrm{W}=[\mathrm{V}] \subset\left[\bigcup_{j=1}^{\infty} \mathcal{W}_{j}\right] \subset \mathrm{W}$, isto é,

$$
\left[\bigcup_{j=1}^{\infty} \mathcal{W}_{j}\right]=\mathrm{W}
$$

Mostremos agora que existe $C, \operatorname{com} 1 \leq C<\infty$, tal que $\left\|\sum_{j=1}^{n} \mathrm{w}_{j}\right\|_{V} \leq C\left\|\sum_{j=1}^{n+m} \mathrm{w}_{j}\right\|_{V}$, $\forall m, n \in \mathbb{N}, \mathrm{e} \mathrm{w}_{j} \in \mathcal{W}_{j}(j=1,2, \ldots, n+m)$.

Caso 1: Seja $\left\|\sum_{j=1}^{n} \mathrm{w}_{j}\right\|_{V}=\sup \left\{\left\|w_{j}\right\|: 1 \leq j \leq n\right\}$.

Como $\sup \left\{\left\|w_{j}\right\|: 1 \leq j \leq n\right\} \leq \sup \left\{\left\|w_{j}\right\|: 1 \leq j \leq n+m\right\} ;$ então $\left\|\sum_{j=1}^{n} \mathrm{w}_{j}\right\|_{V} \leq$ $\sup \left\{\left\|w_{j}\right\|: 1 \leq j \leq n+m\right\} \leq\left\|\sum_{j=1}^{n+m} \mathrm{w}_{j}\right\|_{V}$.

Caso 2: Seja $\left\|\sum_{j=1}^{n} \mathrm{w}_{j}\right\|_{V}=\sup \left\{\left(\sum_{k=1}^{\infty}\left|u_{i k}^{*}\left(\sum_{j=1}^{n} \mathrm{w}_{j}\right)\right|^{2}\right)^{\frac{1}{2}}: i \in N_{1}\right\}$.

$$
\begin{aligned}
& \text { Fixado } i \in N_{1} \text {, teremos }\left(\sum_{k=1}^{\infty}\left|u_{i k}^{*}\left(\sum_{j=1}^{n} \mathrm{w}_{j}\right)\right|^{2}\right)^{\frac{1}{2}}=\left(\sum_{k=1}^{\infty}\left|\sum_{j=1}^{n} u_{i k}^{*}\left(\mathrm{w}_{j}\right)\right|^{2}\right)^{\frac{1}{2}} \\
= & \left(\sum_{k=1}^{n}\left|u_{i k}^{*}\left(\mathrm{w}_{k}\right)\right|^{2}\right)^{\frac{1}{2}} \leq\left(\sum_{k=1}^{m+n}\left|u_{i k}^{*}\left(\mathrm{w}_{k}\right)\right|^{2}\right)^{\frac{1}{2}}=\left(\sum_{k=1}^{\infty}\left|\sum_{j=1}^{n+m} u_{i k}^{*}\left(\mathrm{w}_{j}\right)\right|^{\frac{1}{2}}\right)^{\frac{1}{2}} \\
= & \left(\sum_{k=1}^{\infty}\left|u_{i k}^{*}\left(\sum_{j=1}^{n+m} \mathrm{w}_{j}\right)\right|^{\frac{1}{2}} \operatorname{Logo}, \sup \left\{\left(\sum_{k=1}^{\infty}\left|u_{i k}^{*}\left(\sum_{j=1}^{n} \mathrm{w}_{j}\right)\right|^{2}\right)^{\frac{1}{2}}: i \in N_{1}\right\} \leq\right.
\end{aligned}
$$




$$
\leq \sup \left\{\left(\sum_{k=1}^{\infty}\left|u_{i k}^{*}\left(\sum_{j=1}^{n+m} \mathrm{w}_{j}\right)\right|^{2}\right)^{\frac{1}{2}}: i \in N_{1}\right\} \leq\left\|\sum_{j=1}^{n+m} \mathrm{w}_{j}\right\|_{V}
$$

De maneira análoga, teremos $\left\|\sum_{j=1}^{n} \mathrm{w}_{j}\right\|_{V} \leq\left\|\sum_{j=1}^{n+m} \mathrm{w}_{j}\right\|_{V}$, para os casos

$$
\begin{aligned}
\left\|\sum_{j=1}^{n} \mathrm{w}_{j}\right\|_{V} & =\sup \left\{\left(\sum_{k=1}^{\infty}\left|v_{i k}^{*}\left(\sum_{j=1}^{n} \mathrm{w}_{j}\right)\right|^{2}\right)^{\frac{1}{2}}: i \in N_{2}\right\}, \\
\left\|\sum_{j=1}^{n} \mathrm{w}_{j}\right\|_{V} & =\sup \left\{\left(\sum_{k=1}^{\infty}\left|w_{i k}^{*}\left(\sum_{j=1}^{n} \mathrm{w}_{j}\right)\right|^{2}\right)^{\frac{1}{2}}: i \in N_{3}\right\} \\
\mathrm{e}\left\|\sum_{j=1}^{n} \mathrm{w}_{j}\right\|_{V} & =\sup \left\{\left(\sum_{k=1}^{\infty}\left|v_{i k}^{*}\left(\sum_{j=1}^{n} \mathrm{w}_{j}\right)\right|^{4}+\left|w_{i k}^{*}\left(\sum_{j=1}^{n} \mathrm{w}_{j}\right)\right|^{4}\right)^{\frac{1}{4}}: i \in N_{4}\right\} .
\end{aligned}
$$

Concluímos que existe $C=1$ tal que $\forall m, n \in \mathbb{N}$ e $\mathrm{w}_{j} \in \mathcal{W}_{j}$,

$$
\left\|\sum_{j=1}^{n} \mathrm{w}_{j}\right\|_{V} \leq C\left\|\sum_{j=1}^{n+m} \mathrm{w}_{j}\right\|_{V}
$$

De (1.8) e (1.9), utilizando o Teorema 0.23 , temos que $\left(\mathcal{W}_{j}\right)_{j=1}^{\infty}$ é uma decomposição de Schauder do espaço W. Escreveremos W $=\sum_{j=1}^{\infty} \oplus \mathcal{W}_{j}$.

Observação 1.18 Pela Definição 0.19 segue que, dado $\mathrm{w} \in \mathrm{W}$, existe uma única. seqüência $\left(\mathrm{w}_{j}\right)_{j=1}^{\infty}$ com $\mathrm{w}_{j} \in \mathcal{W}_{j}$ tal que $\mathrm{w}=\sum_{j=1}^{\infty} \mathrm{w}_{j}$.

Observação 1.19 Como conseqüência do lema anterior, temos também que $\|\mathrm{w}\|=$ $\left\|\sum_{j=1}^{\infty} \mathrm{w}_{j}\right\|=\left\|\lim _{n \rightarrow+\infty} \sum_{j=1}^{n} \mathrm{w}_{j}\right\|=\lim _{n \rightarrow+\infty}\left\|\sum_{j=1}^{n} \mathrm{w}_{j}\right\|_{V}$ e como a constante de decomposição $C=1$, segue que $\left\|\sum_{j=1}^{n} \mathrm{w}_{j}\right\|_{V} \leq\left\|\sum_{j=1}^{n+1} \mathrm{w}_{j}\right\|_{V} \quad \forall n \in \mathbb{N}$. Concluímos que $\|\mathrm{w}\|=$ $\sup \left\{\left\|\sum_{j=1}^{n} \mathrm{w}_{j}\right\|_{V}: n \in \mathbb{N}\right\}$, onde $\mathrm{w} \in \mathbf{W}, \mathrm{w}=\sum_{j=1}^{\infty} \mathrm{w}_{j}$.

Além disso, a projeção coordenada $P_{j}$ associada à decomposição $\left(\mathcal{W}_{j}\right)_{j=1}^{\infty}, P_{j}$ : $\mathrm{W} \rightarrow \mathrm{W}$ definida por $P_{j}(\mathrm{w})=\mathrm{w}_{j}$ é, pela Definição 0.21 , limitada para todo $j \in \mathbb{N}$ e também $\left\|P_{j}(\mathrm{w})\right\|_{V}=\left\|\mathrm{w}_{j}\right\|_{V} \leq\left\|\sum_{k=1}^{j} \mathrm{w}_{k}\right\|_{V} \leq\|\mathrm{w}\|$, isto é, $\left\|P_{j}\right\|=1, \forall j \in \mathbb{N}$. 
Observação 1.20 Considerando cada subespaço $\mathcal{W}_{j}$ de $\mathrm{W}$, concluímos que a seqüência $\left(X_{i}\right)_{i=1}^{\infty}$ de cópias do espaço $X$, é também uma decomposição de Schauder de W.

De fato, supondo por exemplo que $i=2 j$ para algum $j \in \mathbb{N}$, a cópia $X_{i}$ de $X$ é isométrica ao subespaço de $\mathrm{V}, X_{2 j}=\left\{\left(0,0, \ldots,\left(0, z_{2 j}\right), 0,0, \ldots\right):\left(0, z_{2 j}\right) \in\right.$ $\mathcal{W}\}$ pela isometria $T: X_{i} \rightarrow X_{2 j}$ definida por $T\left(z_{i}\right)=\left(0,0, \ldots,\left(0, z_{i}\right), 0,0, \ldots\right)$, pois $\left\|T\left(z_{i}\right)\right\|_{V}=\left\|\left(0,0, \ldots,\left(0, z_{i}\right), 0,0, \ldots\right)\right\|_{V}=\left\|z_{i}\right\|$. Assim, para cada $j \in \mathbb{N}$, os subespaços de W, $X_{2 j-1}$ e $X_{2 j}$, são fechados.

Além disso, dado $\mathrm{w} \in \mathrm{W}$, existe uma única seqüência $\left(\mathrm{w}_{j}\right)_{j=1}^{\infty} \operatorname{com} \mathrm{w}_{j} \in \mathcal{W}_{j}$ e $\sum_{j=1}^{\infty} \mathrm{w}_{j}=\mathrm{w}$, mas como $\mathrm{w}_{j} \in \mathcal{W}_{j} \sim X_{2 j-1} \oplus_{\infty} X_{2 j}$, existe um único par $\left(z_{2 j-1}, z_{2 j}\right)$ com $\mathrm{w}_{j}=\left(0,0, \ldots,\left(z_{2 j-1}, z_{2 j}\right), 0,0, \ldots\right)$.

Assim, dado $\mathbf{w} \in \mathbf{W}$, existe uma única seqüência $\left(z_{2 j-1}, z_{2 j}\right)_{j=1}^{\infty} \operatorname{com} z_{2 j-1} \in$ $X_{2 j-1}$ e $z_{2 j} \in X_{2 j}$ tal que $\sum_{j=1}^{\infty}\left(z_{2 j-1}+z_{2 j}\right)=\mathrm{w}$.

Escreveremos simplesmente $\mathbf{W}=\sum_{i=1}^{\infty} \oplus X_{i}$ e $\mathbf{w}=\sum_{i=1}^{\infty} z_{i}, z_{i} \in X_{i}$, observando que a convergência se dá na norma definida em W.

Observação 1.21 As projeções $P_{i}: \mathrm{W} \rightarrow \mathrm{W}$ associadas a decomposição $\left(X_{i}\right)_{i=1}^{\infty}$ de W são, pela Definição 0.21 , limitadas. Além disso, supondo novamente $i=2 j$, temos $\left\|P_{i}(\mathrm{w})\right\|_{V}=\left\|\left(0,0, \ldots,\left(0, z_{2 j}\right), 0,0, \ldots\right)\right\|_{V}=\left\|z_{2 j}\right\| \leq \operatorname{máx}\left\{\left\|z_{2 j-1}\right\|,\left\|z_{2 j}\right\|\right\}=$ $\left\|w_{j}\right\| \leq\left\|\mathrm{w}_{j}\right\|_{V} \leq\left\|\sum_{k=1}^{j} \mathrm{w}_{j}\right\|_{V} \leq\|\mathrm{w}\|$, isto é, $\left\|P_{i}\right\|=1, \forall i \in \mathbb{N}$.

Lema 1.22 Se X é o espaço de Gowers e Maurey e W o espaço de Banach da Definição 1.15, então

(a) $\mathbf{W} \stackrel{c}{\hookrightarrow} X \oplus \mathbf{W} \in X \oplus \mathbf{W} \stackrel{c}{\hookrightarrow} \mathbf{W}$;

(b) $(X \oplus \mathrm{W})^{2} \sim \mathrm{W}$; 
(c) $(X \oplus \mathbf{W})^{3} \sim X \oplus \mathbf{W}$.

Demonstração: (a) $\mathbf{W} \stackrel{c}{\hookrightarrow} X \oplus \mathbf{W}$, pois existe $X<X \oplus \mathbf{W}$, tal que $X \oplus \mathbf{W} \sim X \oplus \mathbf{W}$

$$
\begin{aligned}
\mathrm{W} & =\sum_{j=1}^{\infty} \oplus \mathcal{W}_{j} \sim \mathcal{W}_{1} \oplus \sum_{j=2}^{\infty} \oplus \mathcal{W}_{j} \sim\left(X_{1} \oplus X_{2}\right) \oplus\left(\sum_{j=2}^{\infty} \oplus \mathcal{W}_{j}\right) \\
& \sim X_{1} \oplus\left(X_{2} \oplus \sum_{j=2}^{\infty} \oplus \mathcal{W}_{j}\right)
\end{aligned}
$$

Mostraremos que $\sum_{j=2}^{\infty} \oplus \mathcal{W}_{j} \sim \mathrm{W}$, logo o subespaço de $\mathbf{W}, X_{2} \oplus\left(\sum_{j=2}^{\infty} \oplus \mathcal{W}_{j}\right)$, será isomorfo a $X \oplus \mathbf{W}$, pois $X_{2}$ é cópia isométrica de $X$ e daí teremos $X_{1} \oplus\left(X_{2} \oplus\left(\sum_{j=1}^{\infty} \oplus \mathcal{W}_{j}\right)\right)$ $\sim X \oplus(X \oplus \mathbf{W})$ assim, por $(1.10)$, obteremos $\mathbf{W} \sim X \oplus(X \oplus \mathbf{W})$ e $X \oplus \mathbf{W} \stackrel{c}{\hookrightarrow} \mathbf{W}$.

Para mostrarmos que $\mathrm{W} \sim \sum_{j=2}^{\infty} \oplus \mathcal{W}_{j}$, observemos que $\sum_{j=2}^{\infty} \oplus \mathcal{W}_{j}$ é a decomposição de Schauder de um subespaço fechado $\mathbf{W}_{0}$ de $\mathbf{W}\left(\mathbf{W} \sim \mathcal{W}_{1} \oplus \mathbf{W}_{0}\right)$ e que dado $\mathrm{w} \in \mathrm{W}_{0}$, existe uma única seqüência $\left(\mathrm{w}_{j}\right)_{j=1}^{\infty}$ com $\mathrm{w}_{1}=0$ tal que $\mathrm{w}=\sum_{j=1}^{\infty} \mathrm{w}_{j}$.

Associamos a $\mathrm{w}$ a seqüência $\left(0, w_{2}, w_{3}, \ldots, w_{j}, w_{j+1}, \ldots\right)$, com $\|\mathrm{w}\|=$ $\sup \left\{\left\|\sum_{j=1}^{n} \mathrm{w}_{j}\right\|_{V}: n \in \mathbb{N}\right\}=\sup \left\{\left\|\left(0, w_{2}, w_{3}, \ldots, w_{j}, 0,0, \ldots\right)\right\|_{V}: j \in \mathbb{N}\right\}$.

Definimos então, a aplicação linear $T: \mathbf{W} \rightarrow \mathbf{W}_{0}$ por $T(\mathbf{w})=T\left(w_{1}, w_{2}, \ldots, w_{j}\right.$, $\ldots)=\left(0, w_{1}, w_{2}, \ldots, w_{j}, \ldots\right)$.

A aplicação $T$ está bem definida, pois $T(\mathbf{w})=\left(0, w_{1}, w_{2}, \ldots, w_{j}, \ldots\right)$ e $\sum_{j=1}^{\infty} \mathbf{w}_{j}$ converge, já que $\mathbf{w}=\left(w_{1}, w_{2}, \ldots, w_{j}, \ldots\right) \in \mathbf{W}$.

$T$ é isometria, pois $\|T(\mathbf{w})\|=\left\|T\left(w_{1}, w_{2}, \ldots, w_{j}, \ldots\right)\right\|=\left\|\left(0, w_{1}, w_{2}, \ldots, w_{j}, \ldots\right)\right\|$ $=\sup \left\{\|\left(0, w_{1}, w_{2}, \ldots, w_{j}, 0,0, \ldots \|_{V}: j \in \mathbb{N}\right\}=\sup \left\{\left\|\left(w_{1}, w_{2}, \ldots, w_{j}, 0,0, \ldots\right)\right\|_{V}:\right.\right.$ $j \in \mathbb{N}\}=\|w\|$.

Finalizando, dado $\mathrm{z} \in \mathbf{W}_{0}, \mathbf{z}=\left(0, w_{2}, w_{3}, \ldots, w_{j}, \ldots\right)$, temos que $\mathrm{z}=\sum_{j=2}^{\infty} \mathrm{w}_{j}$ converge, logo existe $\mathbf{x}=\left(w_{2}, w_{3}, \ldots, w_{j}, \ldots\right), \mathbf{x} \in \mathbf{W}$, tal que $T(\mathbf{x})=\mathbf{z}$. 
Assim, $T$ é isometria sobre $\mathbf{W}_{0}$ e $\mathbf{W} \sim \sum_{j=2}^{\infty} \oplus \mathcal{W}_{j}$

(b) $X \oplus \mathrm{W} \sim X_{1} \oplus \mathrm{W}$ e $X \oplus \mathrm{W} \sim X_{2} \oplus \mathbf{W}$, logo

$$
(X \oplus \mathbf{W})^{2} \sim\left(X_{1} \oplus \mathbf{W}\right) \underset{\infty}{\oplus}\left(X_{2} \oplus \mathbf{W}\right) .
$$

Mostremos inicialmente que $\mathbf{W} \sim\left(\sum_{j=1}^{\infty} \oplus \mathcal{W}_{2 j+1}\right)$ e $\mathbf{W} \sim\left(\sum_{j=1}^{\infty} \oplus \mathcal{W}_{2 j}\right)$.

Observemos que $\sum_{j=1}^{\infty} \oplus \mathcal{W}_{2 j}$ é a decomposição de Schauder de um subespaço fechado $\mathbf{W}_{2}$ de $\mathbf{W}\left(\mathbf{W} \sim\left(\sum_{j=1}^{\infty} \oplus \mathcal{W}_{2 j-1}\right) \oplus \mathbf{W}_{2}\right)$ e dado $\mathbf{w} \in \mathbf{W}_{2}$, existe uma única seqüência $\left(\mathrm{w}_{j}\right)_{j=1}^{\infty}$ com $\mathrm{w}_{2 j-1}=0$, tal que $\mathrm{w}=\sum_{j=1}^{\infty} \mathrm{w}_{j}$.

Identificamos $\mathbf{w}$ com a seqüência $\left(0, w_{2}, 0, w_{4}, 0, w_{6}, \ldots, 0, w_{2 j}, 0, \ldots\right)$ e $\|\mathbf{w}\|=$ $\sup \left\{\left\|\sum_{j=1}^{n} \mathrm{w}_{j}\right\|_{V}: n \in \mathbb{N}\right\}=\sup \left\{\left\|\left(0, w_{2}, 0, w_{4}, 0, \ldots, 0, w_{2 j}, 0, \ldots\right)\right\|_{V}: j \in \mathbb{N}\right\}$.

Dado $\mathbf{w} \in \mathbf{W}, \mathbf{w}=\left(w_{1}, w_{2}, \ldots, w_{j}, \ldots\right)$, definimos a aplicação linear $T: \mathbf{W} \rightarrow$ $\mathbf{W}_{2}$ por $T(\mathbf{w})=\left(0, w_{1}, 0, w_{2}, \ldots, 0, w_{j}, \ldots\right)$.

A aplicação $T$ está bem definida, pois $T(\mathbf{w})=\left(0, w_{1}, 0, w_{2}, \ldots, 0, w_{j}, 0, \ldots\right)$ e $\sum_{j=1}^{\infty} \mathbf{w}_{j}$ converge, já que $\mathrm{w}=\left(w_{1}, w_{2}, \ldots, w_{j}, \ldots\right) \in \mathrm{W}$.

A aplicação $T$ é isometria, pois $\|T(\mathbf{w})\|=\left\|T\left(w_{1}, w_{2}, \ldots, w_{j}, \ldots\right)\right\|=\|\left(0, w_{1}, 0, w_{2}\right.$, $\left.0, \ldots, 0, w_{j}, \ldots\right) \|=\sup \left\{\left\|\left(0, w_{1}, 0, w_{2}, 0, \ldots, 0, w_{j}, 0,0, \ldots\right)\right\|_{V: j \in \mathbb{N}}\right\}=\sup \left\{\|\left(w_{1}, w_{2}\right.\right.$, $\left.\left.\ldots, w_{j}, 0,0, \ldots\right) \|_{V: j \in \mathbb{N}}\right\}=\|\mathrm{w}\|$.

Temos também que se $\mathbf{z} \in \mathbf{W}_{2}, \mathbf{z}=\left(0, w_{2}, 0, w_{4}, 0, \ldots, 0, w_{2 j}, \ldots\right), \mathbf{z}=\sum_{j=1}^{\infty} \mathbf{w}_{2 j}$ converge, segue que existe $\mathrm{x}=\left(w_{2}, w_{4}, \ldots, w_{2 j}, \ldots\right), \mathrm{x} \in \mathbf{W}$, tal que $T(\mathrm{x})=\mathrm{z}$.

Assim, $T$ é isometria sobre $\mathbf{W}_{2}$ e $\mathbf{W} \sim \sum_{j=1}^{\infty} \oplus \mathcal{W}_{2 j}$.

Considerando $\sum_{j=1}^{\infty} \oplus \mathcal{W}_{2 j+1}$ a decomposição de Schauder do subespaço $\mathbf{W}_{3}$ de 
W e definindo a aplicação linear $T: \mathbf{W} \rightarrow \mathbf{W}_{3}$ por $T\left(w_{1}, w_{2}, \ldots, w_{j}, \ldots\right)=$ $\left(0,0, w_{1}, 0, w_{2}, 0, \ldots, w_{j}, \ldots\right)$, concluímos, utilizando raciocínio análogo ao caso anterior, que $\mathrm{W} \sim \mathrm{W}_{3}$.

$$
\begin{aligned}
& \text { Agora, de (1.11), teremos }(X \oplus \mathbf{W})^{2} \sim\left(X_{1} \oplus\left(\sum_{j=1}^{\infty} \oplus \mathcal{W}_{2 j+1}\right)\right) \oplus\left(X_{2} \oplus\left(\sum_{j=1}^{\infty} \oplus \mathcal{W}_{2 j}\right)\right) \\
& \sim\left(X_{1} \oplus X_{2} \oplus\left(\sum_{j=1}^{\infty} \oplus \mathcal{W}_{2 j+1}\right)\right) \underset{\infty}{\oplus}\left(\sum_{j=1}^{\infty} \oplus \mathcal{W}_{2 j}\right) \sim\left(\mathcal{W}_{1} \oplus\left(\sum_{j=1}^{\infty} \oplus \mathcal{W}_{2 j+1}\right)\right) \underset{\infty}{\oplus}\left(\sum_{j=1}^{\infty} \oplus \mathcal{W}_{2 j}\right) \\
& \sim\left(\sum_{j=1}^{\infty} \oplus \mathcal{W}_{2 j-1}\right) \underset{\infty}{\oplus}\left(\sum_{j=1}^{\infty} \oplus \mathcal{W}_{2 j}\right) \sim\left(\sum_{j=1}^{\infty} \oplus \mathcal{W}_{2 j-1}\right) \oplus\left(\sum_{j=1}^{\infty} \oplus \mathcal{W}_{2 j}\right) \sim \mathrm{W} .
\end{aligned}
$$

(c) $(X \oplus \mathbf{W})^{3} \sim(X \oplus \mathbf{W})^{2} \oplus_{\infty}(X \oplus \mathbf{W}) \sim \mathbf{W} \oplus \underset{\infty}{ }(X \oplus \mathbf{W}) \sim \mathrm{W} \oplus(\mathbf{W} \oplus X) \sim$ $(\mathrm{W} \oplus \mathbf{W}) \oplus X \sim \mathrm{W} \oplus X \sim X \oplus \mathbf{W}$.

$$
\text { Observemos que } \mathbf{W} \oplus \mathbf{W} \sim\left(\sum_{j=1}^{\infty} \oplus \mathcal{W}_{2 j-1}\right) \oplus\left(\sum_{j=1}^{\infty} \oplus \mathcal{W}_{2 j}\right) \sim \mathrm{W}
$$

A partir deste lema concluímos que $\mathbf{W}$ e $X \oplus \mathbf{W}$ são espaços de Banach, tais que $\mathbf{W} \stackrel{c}{\hookrightarrow} X \oplus \mathbf{W}$ e $X \oplus \mathbf{W} \stackrel{c}{\hookrightarrow} \mathbf{W}$.

Nosso principal objetivo é mostrar que $\mathbf{W} \nsucc X \oplus \mathbf{W}$. Observemos que se $Z=$ $X \oplus \mathbf{W}$, o contra-exemplo para o problema de Schroeder-Bernstein será dado por $Z=X \oplus \mathbf{W}$ e $Z^{2} \sim \mathbf{W}$ (pelo item (b) do lema anterior). Teremos também, $Z \sim Z^{3}$ (pelo item (c) do mesmo lema). 


\section{Capítulo 2}

\section{Uma solução para o problema de Schroeder-Bernstein para espaços de Banach}

Neste capítulo mostraremos que o par de espaços de Banach W e $X \oplus \mathbf{W}$, definidos no capítulo anterior, é contra-exemplo para o problema de SchroederBernstein.

Novamente, para facilitar a leitura, apresentaremos a seguir a estrutura dos resultados deste capítulo.

Apoiados na propriedade do Lema 1.7 do Capítulo 1, será possível a cada operador $T \in B(\mathbf{W})$ associar uma única matriz infinita de números reais, $\Lambda_{T} \in M_{\infty}(\mathbb{R})$ e um único operador $T_{\Lambda} \in c_{0}$, de tal forma que $T_{\Lambda}(a)=\Lambda_{T} a, \forall a \in c_{00}$. Indicaremos por $\Phi: B(\mathbf{W}) \rightarrow B\left(c_{0}\right)$ esta associação, isto é, $\Phi(T)=T_{\Lambda}$.

Mostraremos no Lema 2.18 que $\Phi$ é um homomorfismo sobre a álgebra dos operadores de W. No Lema 2.21 mostraremos que, se $\Phi(T)=T_{\Lambda}$ é um operador de Fredholm, então $i\left(T_{\Lambda}\right)$ é par.

No resultado principal (Teorema 2.22), iremos supor que exista $T: \mathbf{W} \rightarrow X \oplus \mathbf{W}$, 
$T$ um isomorfismo e concluiremos que, neste caso, $\Phi(T)=T_{\Lambda}$ é um operador de Fredholm com $i\left(T_{\Lambda}\right)$ ímpar, logo $\mathbf{W} \not X \oplus \mathbf{W}$.

A teoria apresentada neste capítulo pode ser encontrada em [G].

Provaremos inicialmente um lema, que será utilizado posteriormente por razões essencialmente "técnicas".

Lema 2.1 Se $\mathrm{w}_{1}, \mathrm{w}_{2}, \ldots, \mathrm{w}_{N}$ é uma seqüência de vetores sucessivos de $\mathrm{W}$, então

$$
\left\|\sum_{k=1}^{N} \mathrm{w}_{k}\right\|_{V} \leq\left(\sum_{k=1}^{N}\left\|\mathrm{w}_{k}\right\|_{V}^{2}\right)^{\frac{1}{2}}
$$

Demonstração: Observemos antes da demonstração, que os vetores $\mathrm{w}_{k}$ no enunciado do lema, não são necessariamente os vetores $\mathrm{w}_{k}=\left(0,0, \ldots, w_{k}, 0, \ldots\right) \in \mathcal{W}_{k}$, definidos anteriormente na decomposição de Schauder de $W$, mas sim quaisquer vetores de suporte finito de W. Seja $\sum_{k=1}^{N} \mathbf{w}_{k}=\left(w_{1}, w_{2}, \ldots, w_{j}, \ldots\right)$.

Caso 1: $\left\|\sum_{k=1}^{N} \mathrm{w}_{k}\right\|_{V}=\sup \left\{\left\|w_{j}\right\|: j \in \mathbb{N}\right\}$.

Neste caso, $\sup \left\{\left\|w_{j}\right\|: j \in \mathbb{N}\right\} \leq \sup \left\{\left\|P_{j}\left(\sum_{k=1}^{N} \mathrm{w}_{k}\right)\right\|_{V}: j \in \mathbb{N}\right\}$.

Como $\mathrm{w}_{1}<\mathrm{w}_{2}<\cdots<\mathrm{w}_{k}$, existirá $k_{0}$ com $1 \leq k_{0} \leq N$ e $j_{0} \in \mathbb{N}$, tais que $\sup \left\{\left\|P_{j}\left(\sum_{k=1}^{N} \mathrm{w}_{k}\right)\right\|_{V}: j \in \mathbb{N}\right\}=\left\|P_{j_{0}}\left(\mathrm{w}_{k_{0}}\right)\right\|_{V} \leq\left\|\mathrm{w}_{k_{0}}\right\|_{V}=\left(\left\|\mathrm{w}_{k_{0}}\right\|_{V}^{2}\right)^{\frac{1}{2}}$

$$
\leq\left(\sum_{k=1}^{N}\left\|\mathrm{w}_{k}\right\|_{V}^{2}\right)^{\frac{1}{2}}
$$

Caso 2: Seja $\left\|\sum_{k=1}^{N} \mathrm{w}_{k}\right\|_{V}=\sup \left\{\left(\sum_{j=1}^{\infty}\left|u_{i j}^{*}\left(\sum_{k=1}^{N} \mathrm{w}_{k}\right)\right|^{2}\right)^{\frac{1}{2}}: i \in N_{1}\right\}$.

Fixados $i \in N_{1}$ e $j \in \mathbb{N}$, teremos $\left|u_{i j}^{*}\left(\sum_{k=1}^{N} \mathrm{w}_{k}\right)\right|^{2}=\left|\sum_{k=1}^{N} u_{i j}^{*}\left(\mathrm{w}_{k}\right)\right|^{2}$. 


$$
\begin{aligned}
& \text { Como } \mathrm{w}_{1}<\mathrm{w}_{2}<\cdots<\mathrm{w}_{N},\left|\sum_{k=1}^{N} u_{i j}^{*}\left(\mathrm{w}_{k}\right)\right|^{2}=\left|u_{i j}^{*}\left(\mathrm{w}_{k}\right)\right|^{2} \text { para um único } k \\
& \text { tal que } P_{j}\left(\mathrm{w}_{k}\right) \neq 0 \text { ou }\left|\sum_{k=1}^{N} u_{i j}^{*}\left(\mathrm{w}_{k}\right)\right|^{2}=0 \text {, se } P_{j}\left(\mathrm{w}_{k}\right)=0,1 \leq k \leq N \text {. Assim, } \\
& \sum_{j=1}^{\infty}\left|u_{i j}^{*}\left(\sum_{k=1}^{N} \mathrm{w}_{k}\right)\right|^{2}=\sum_{j=1}^{\infty}\left|\sum_{k=1}^{N} u_{i j}^{*}\left(\mathrm{w}_{k}\right)\right|^{2}=\sum_{k=1}^{N}\left(\sum_{j=1}^{\infty}\left|u_{i j}^{*}\left(\mathrm{w}_{k}\right)\right|^{2}\right) \leq \sum_{k=1}^{N}\left\|\mathrm{w}_{k}\right\|_{V}^{2} \text {. } \\
& \text { Concluímos que }\left(\sum_{j=1}^{\infty}\left|u_{i j}^{*}\left(\sum_{k=1}^{N} \mathrm{w}_{k}\right)\right|^{2}\right)^{\frac{1}{2}} \leq\left(\sum_{k=1}^{N}\left\|\mathrm{w}_{k}\right\|_{V}^{2}\right)^{\frac{1}{2}} \text { para todo } i \in N_{1} \text {. Lo- } \\
& \text { go, }\left\|\sum_{k=1}^{N} \mathrm{w}_{k}\right\|_{V} \leq\left(\sum_{k=1}^{N}\left\|\mathrm{w}_{k}\right\|_{V}^{2}\right)^{\frac{1}{2}} .
\end{aligned}
$$

De maneira análoga, obtemos a desigualdade ao trocarmos $u_{i j}^{*}$ por $v_{i j}^{*}$ ou $w_{i j}^{*}$ no valor de $\left\|\sum_{k=1}^{N} \mathrm{w}_{k}\right\|_{V}$.

Definição 2.2 Sejam $P_{i}: \mathrm{W} \rightarrow \mathrm{W}$ as projeções coordenada associadas à decomposição $\left(X_{i}\right)_{i=1}^{\infty}$ de $\mathbf{W}$ e $T: \mathbf{W} \rightarrow \mathbf{W}$ um operador limitado. Definimos para cada $i, j \in \mathbb{N}$ o operador $T_{i j}: X_{j} \rightarrow X_{i}$ como sendo a restrição de $P_{i} T$ em $X_{j}$, isto é, $T_{i j}=\left.P_{i} T\right|_{X_{j}}$

Observação $2.3 T_{i j}$ é limitado para todo $i, j \in \mathbb{N}$ pois $T \in B(\mathbf{W})$ e $P_{i}$ é limitado para todo $i \in \mathbb{N}$ (pela Observação 1.21).

Pelo Lema 1.7, para cada $i, j \in \mathbb{N}$ existe $\lambda_{i j} \in \mathbb{R}$, tal que $\lim _{n \rightarrow+\infty} T_{i j}\left(x_{n j}\right)-\lambda_{i j} x_{n i}=$ 0 , onde $\left(x_{n i}\right)_{n=1}^{\infty}$ indica a cópia da seqüência de $X,\left(x_{n}\right)_{n=1}^{\infty}$ do Lema 1.7 , em cada. $X_{i}$.

Observação 2.4 Para cada $i, j \in \mathbb{N}, \lambda_{i j} \in \mathbb{R}$ é único, pois se $\lambda_{i j}$ e $\beta_{i j}$ são tais que $\lim _{n \rightarrow+\infty} T_{i j} x_{n j}-\lambda_{i j} x_{n i}=0$ e $\lim _{n \rightarrow+\infty} T_{i j} x_{n j}-\beta_{i j} x_{n i}=0$, então $\lim _{n \rightarrow+\infty}\left(\beta_{i j}-\lambda_{i j}\right) x_{n i}=0$ segue que $\lim _{n \rightarrow+\infty}\left|\beta_{i j}-\lambda_{i j}\right|\left\|x_{n i}\right\|=0, \operatorname{logo} \lim _{n \rightarrow+\infty}\left|\beta_{i j}-\lambda_{i j}\right|=0$ e conseqüentemente $\lambda_{i j}=\beta_{i j}$. 
Definição 2.5 Dado $T \in B(\mathrm{~W})$ pela observação anterior, podemos definir uma aplicação $\phi: B(\mathbf{W}) \rightarrow M_{\infty}(\mathbb{R})$ por $\phi(T)=\left(\lambda_{i j}\right)_{i, j=1}^{\infty}$, onde o termo geral $\lambda_{i j} \in \mathbb{R}$ é tal que $\lim _{n \rightarrow+\infty} T_{i j}\left(x_{n j}\right)-\lambda_{i j} x_{n i}=0$ e $M_{\infty}(\mathbb{R})$ é o espaço vetorial das matrizes infinitas sobre IR.

Escreveremos $\phi(T)=\Lambda_{T}=\left(\lambda_{i j}\right)_{i, j=1}^{\infty}$, para representar esta matriz associada ao operador $T \in B(\mathbf{W})$.

Observação $2.6 \phi: B(\mathbf{W}) \rightarrow M_{\infty}(\mathbb{R})$ é uma aplicação linear. De fato, sejam $T, S \in B(\mathbf{W}), \Lambda_{T}=\left(\lambda_{i j}\right)_{i, j=1}^{\infty}$ e $\Lambda_{S}=\left(\beta_{i j}\right)_{i, j=1}^{\infty}$ as matrizes associadas aos operadores $T$ e $S$.

Para todo $i, j \in \mathbb{N}$, teremos $\lim _{n \rightarrow+\infty} T_{i j}\left(x_{n j}\right)-\lambda_{i j} x_{n i}=0 \mathrm{e} \lim _{n \rightarrow+\infty} S_{i j}\left(x_{n j}\right)-\beta_{i j} x_{n i}=$ 0. Logo, $\lim _{n \rightarrow+\infty} T_{i j}\left(x_{n j}\right)+S_{i j}\left(x_{n j}\right)-\left(\lambda_{i j}+\beta_{i j}\right) x_{n i}=0$, então $\lim _{n \rightarrow+\infty}\left(T_{i j}+S_{i j}\right)\left(x_{n j}\right)-$ $\left(\lambda_{i j}+\beta_{i j}\right) x_{n i}=0$, portanto $\lim _{n \rightarrow+\infty}(T+S)_{i j}\left(x_{n j}\right)-\left(\lambda_{i j}+\beta_{i j}\right) x_{n i}=0$, isto é, $\phi(T+S)=$ $\Lambda_{T+S}=\left(\lambda_{i j}+\beta_{i j}\right)_{i, j=1}^{\infty}=\Lambda_{T}+\Lambda_{S}=\phi(T)+\phi(S)$.

Além disso, dado $\gamma \in \mathbb{R}, \lim _{n \rightarrow+\infty} T_{i j}\left(x_{n j}\right)-\lambda_{i j} x_{n i}=0, \log 0 \lim _{n \rightarrow+\infty} \gamma\left[T_{i j}\left(x_{n j}\right)-\right.$ $\left.\lambda_{i j} x_{n i}\right]=0$, portanto $\lim _{n \rightarrow+\infty}\left(\gamma T_{i j}\right)\left(x_{n j}\right)-\left(\gamma \lambda_{i j}\right) x_{n i}=0, \quad$ conseqüentemente $\lim _{n \rightarrow+\infty}(\gamma T)_{i j}\left(x_{n j}\right)-\left(\gamma \lambda_{i j}\right) x_{n i}=0$, isto é, $\phi(\gamma T)=\Lambda_{\gamma T}=\left(\gamma \lambda_{i j}\right)_{i, j=1}^{\infty}=\gamma \Lambda_{T}=\gamma \phi(T)$.

Mostraremos a seguir que a cada operador $T \in B(\mathrm{~W})$ podemos associar, a partir de uma aplicação que denominaremos de $\Phi$, um único operador $T_{\Lambda} \in c_{0}$, tal que $T_{\Lambda}(a)=\phi(T)(a)=\Lambda_{T} a, \forall a \in c_{00}$, onde $\Lambda_{T}=\left(\lambda_{i j}\right)_{i, j}^{\infty}$ é a matriz associada ao operador $T$.

Nosso objetivo imediato será mostrar que $\Phi: \mathrm{W} \rightarrow c_{0}$ é na verdade um homomorfismo de álgebra; para isso precisaremos dos lemas que virão a seguir $(2.10,2.11$, $2.12,2.14$ e 2.16). Antes porém, daremos a definição de um produto que utilizaremos nesses lemas. 
Definição 2.7 Sejam $S=\left\{\left(a_{i}\right)_{i=1}^{\infty}, a_{i} \in \mathbb{R}\right\}$ o espaço vetorial das seqüências de números reais e $X$ o espaço de Gowers e Maurey. Dados $a \in S$ e $z \in X$, definimos o produto $a \otimes z$ pelo vetor $\left(a_{1} z, a_{2} z, \ldots, a_{n} z, \ldots\right)$.

Escreveremos $(a \otimes z)_{i}$, para representar a projeção do vetor em cada cópia $X_{i}$ de $X$, isto é, $(a \otimes z)_{i}=a_{i} z_{i}$ e $(a \otimes z)_{j}$ para representar a projeção do vetor em $\mathcal{W}_{j}$, isto é, $(a \otimes z)_{j}=a_{2 j-1} z_{2 j-1}+a_{2 j} z_{2 j}$. Assim, representaremos também o vetor $a \otimes z$, a partir da seqüência: $\left(a_{1} z_{1}, a_{2} z_{2}, \ldots, a_{i} z_{i}, \ldots\right)$ onde $z_{i}$ é cópia de $z \in X$, em cada cópia $X_{i}$.

Observação 2.8 Se $a \otimes z \in \mathbf{W}$, escreveremos também $a \otimes z=\sum_{i=1}^{\infty} a_{i} z_{i}$ e $(a \otimes z)_{i}=$ $P_{i}(a \otimes z)$, onde $P_{i}$ são as projeções associadas à decomposição $\left(X_{i}\right)_{i=1}^{\infty}$ de $\mathbf{W}$.

Analogamente, se $a \otimes z \in \mathrm{W}$, escreveremos também $a \otimes z=\sum_{j=1}^{\infty} \mathrm{w}_{j} \mathrm{e}(a \otimes z)_{j}=$ $P_{j}(a \otimes z)$, onde $P_{j}$ são as projeções associadas à decomposição $\left(\mathcal{W}_{j}\right)_{j=1}^{\infty}$ de $\mathbf{W}$.

Observação 2.9 Se $a \in c_{00}$, então $a \otimes z \in \mathrm{W}$, pois $a \otimes z \in \mathrm{V}$, já que $\operatorname{supp}(a)$ é finito.

Lema 2.10 Se $a \in c_{00}, T \in B(\mathbf{W})$ e $\Lambda_{T}=\left(\lambda_{k, j}\right)_{k, j}^{\infty}$ é a matriz associada a $T$, então a seqüência $T\left(a \otimes x_{n}\right)-\left(\Lambda_{T} a\right) \otimes x_{n}$ converge pontualmente a zero, isto é, $\lim _{n \rightarrow+\infty}\left(T\left(a \otimes x_{n}\right)\right)_{i}-\left(\Lambda_{T} a \otimes x_{n}\right)_{i}=0$.

Demonstração: Seja $a \in c_{00}$ com máx $\operatorname{supp}(a)=p$, então $T\left(a \otimes x_{n}\right)=T\left(a_{1} x_{n 1}+\right.$ $\left.a_{2} x_{n 2}+\cdots+a_{p} x_{n p}\right)=\sum_{j=1}^{p} a_{j} T\left(x_{n j}\right), \quad \forall n \in \mathbb{N}$.

$$
\text { Assim, }\left(T\left(a \otimes x_{n}\right)\right)_{i}=\sum_{j=1}^{p} a_{j}\left(T\left(x_{n j}\right)\right)_{i}=\sum_{j=1}^{p} a_{j} T_{i j}\left(x_{n j}\right) .
$$

Por outro lado, $\Lambda_{T} a=\left(\lambda_{11} a_{1}+\lambda_{12} a_{2}+\cdots+\lambda_{1 p} a_{p}, \lambda_{21} a_{1}+\cdots+\lambda_{2 p} a_{p}, \ldots, \lambda_{k 1} a_{1}+\right.$ $\left.\cdots+\lambda_{k p} a_{p}, \ldots\right)=\left(\sum_{j=1}^{p} \lambda_{k j} a_{j}\right)_{k=1}^{\infty} \mathrm{e}\left(\Lambda_{T} a \otimes x_{n}\right)=\left(\sum_{j=1}^{p} \lambda_{k j} a_{j} x_{n k}\right)_{k=1}^{\infty}, \forall n \in \mathbb{N}$. 
Portanto, $\left(\Lambda_{T} a \otimes x_{n}\right)_{i}=\sum_{j=1}^{p} \lambda_{i j} a_{j} x_{n i}$.

Assim, fixado $i \in \mathbb{N},\left(T\left(a \otimes x_{n}\right)\right)_{i}-\left(\Lambda_{T} a \otimes x_{n}\right)_{i}=\sum_{j=1}^{p} a_{j} T_{i j}\left(x_{n j}\right)-\sum_{j=1}^{p} \lambda_{i j} a_{j} x_{n i}=$ $\sum_{j=1}^{p} a_{j}\left[T_{i j}\left(x_{n j}\right)-\lambda_{i j} x_{n i}\right]$, para todo $n \in \mathbb{N}$.

Como $\lim _{n \rightarrow+\infty} T_{i j}\left(x_{n j}\right)-\lambda_{i j} x_{n i}=0$, então $\lim _{n \rightarrow+\infty} a_{j}\left[T_{i j}\left(x_{n j}\right)-\lambda_{i j} x_{n i}\right]=0$, para cada $j, 1 \leq j \leq p$

Podemos concluir então que

$$
\lim _{n \rightarrow+\infty} \sum_{j=1}^{p} a_{j}\left[T_{i j}\left(x_{n j}\right)-\lambda_{i j} x_{n i}\right]=0 .
$$

Lema 2.11 (a) Se $c \in c_{0}$ e $n \in N_{0}$, então $c \otimes x_{n} \in \mathrm{W} e\left\|c \otimes x_{n}\right\|=\|c\|_{\infty}$.

(b) Se $a \in c_{00}$ e $n \in N_{4}$, então $\left\|a \otimes x_{n}\right\|_{V}=\|a\|_{4}$.

Demonstração: (a) Sejam $c \in c_{0}, c=\left(c_{i}\right)_{i=1}^{\infty}$ e $n \in N_{0}$. Mostremos que $c \otimes x_{n}=$ $\left(c_{1} x_{n 1}, c_{2} x_{n 2}, \ldots, c_{i} x_{n i}, \ldots\right)$ é um vetor de $\mathbf{W}$, isto é, que $\sum_{i=1}^{\infty} c_{i} x_{n i}$ converge na norma definida em $\mathrm{V}$.

Consideremos a seqüência das somas parciais $S_{k}=\sum_{i=1}^{k} c_{i} x_{n i}$. Sejam $l, k \in \mathbb{N}$, $\operatorname{com} l>k, \log 0\left\|S_{l}-S_{k}\right\|_{V}=\left\|\sum_{i=1}^{l} c_{i} x_{n i}-\sum_{i=1}^{k} c_{i} x_{n i}\right\|_{V}=\| c_{k+1} x_{n k+1}+c_{k+2} x_{n k+2}+$ $\cdots+c_{l} x_{n l} \|_{V}$.

Observemos que se $n \in N_{0}$ e $i \in N_{1}$, então para todo $j \in \mathbb{N}, u_{i j}^{*}\left(c_{2 j-1} x_{n 2 j-1}, c_{2 j} x_{n 2 j}\right)$ $=\left(x_{i}^{*},-x_{i}^{*}\right)\left(c_{2 j-1} x_{n 2 j-1}, c_{2 j} x_{n 2 j}\right)=c_{2 j-1} x_{i}^{*}\left(x_{n 2 j-1}\right)-c_{2 j} x_{i}^{*}\left(x_{n 2 j}\right)=c_{2 j-1} 0-c_{2 j} 0=0$; pois $N_{0} \cap N_{1}=\emptyset$.

O mesmo ocorrerá com $v_{i j}^{*}$ se $i \in N_{2}, w_{i j}^{*}$ se $i \in N_{3}$ e também com $v_{i j}^{*}$ e $w_{i j}^{*}$ se $i \in N_{4}$. Assim, $\left\|S_{l}-S_{k}\right\|_{V}=\operatorname{máx}\left\{\left\|c_{i} x_{n i}\right\|: k+1 \leq i \leq l\right\}=\operatorname{máx}\left\{\left|c_{i}\right|\left\|x_{n i}\right\|:\right.$ $k+1 \leq i \leq l\}=\operatorname{máx}\left\{\left|c_{i}\right|: k+1 \leq i \leq l\right\}$, mas $\lim _{i \rightarrow+\infty}\left|c_{i}\right|=0, \operatorname{logo}\left\|S_{l}-S_{k}\right\|_{V} \rightarrow 0$ se $l, k \rightarrow+\infty$, isto é, $\sum_{i=1}^{\infty} c_{i} x_{n i}$ converge e $c \otimes x_{n} \in \mathrm{W}$. 
Pela Observação 1.19, temos $\left\|c \otimes x_{n}\right\|=\left\|\sum_{i=1}^{\infty} c_{i} x_{n i}\right\|=\sup \left\{\left\|\sum_{i=1}^{k} c_{i} x_{n i}\right\|_{V}: k \in \mathbb{N}\right\}=$ $\sup \left\{\left\|c_{i} x_{n i}\right\|: i \in \mathbb{N}\right\}=\sup \left\{\left|c_{i}\right|: i \in \mathbb{N}\right\}=\|c\|_{\infty}$.

(b) Sejam $n_{0} \in N_{4}$ e $a \in c_{00} \operatorname{com} \operatorname{máx} \operatorname{supp}(a)=p$. Como $n_{0} \in N_{4}$, pela definição de norma em $\mathbf{V}$, se $i \in N_{1}$, então para todo $j \in \mathbb{N}$, temos $u_{i j}^{*}\left(\mathrm{w}_{j}\right)=u_{i j}^{*}\left(a_{2 j-1} x_{n_{0} 2 j-1}+\right.$ $\left.a_{2 j} x_{n_{0} 2 j}\right)=\left(x_{i}^{*},-x_{i}^{*}\right)\left(a_{2 j-1} x_{n_{0} 2 j-1}+a_{2 j} x_{n_{0} 2 j}\right)=a_{2 j-1} x_{i}^{*}\left(x_{n_{0} 2 j-1}\right)-a_{2 j} x_{i}^{*}\left(x_{n_{0} 2 j}\right)=$ $a_{2 j-1} 0-a_{2 j} 0=0$, pois $N_{4} \cap N_{1}=\emptyset$.

O mesmo ocorrerá com $v_{i j}^{*}$ se $i \in N_{2}, w_{i j}^{*}$ se $i \in N_{3}$ e também com $v_{i j}^{*}$ e $w_{i j}^{*}$ se $i \in N_{4} \operatorname{com} i \neq n_{0}$. Assim, $\left\|a \otimes x_{n_{0}}\right\|_{V}=\left\|\sum_{i=1}^{p} a_{i} x_{n_{0} i}\right\|_{V}=\operatorname{máx}\left\{\left\|a_{i} x_{n_{0} i}\right\|: 1 \leq i \leq p\right\} \vee$ $\sup \left\{\left(\sum_{j=1}^{\infty}\left|v_{i j}^{*}\left(a \otimes x_{n_{0}}\right)\right|^{4}+\left|w_{i j}^{*}\left(a \otimes x_{n_{0}}\right)\right|^{4}\right)^{\frac{1}{4}}: i \in N_{4}\right\}=\operatorname{máx}\left\{\left|a_{i}\right|: 1 \leq i \leq p\right\} \vee$ $\left(\left|x_{n_{0}}^{*}\left(a_{1} x_{n_{0} 1}\right)\right|^{4}+\left|x_{n_{0}}^{*}\left(a_{2} x_{n_{0} 2}\right)\right|^{4}+\cdots+\left|x_{n_{0}}^{*}\left(a_{p} x_{n_{0} p}\right)\right|^{4}\right)^{\frac{1}{4}}=\operatorname{máx}\left\{\left|a_{i}\right|: 1 \leq i \leq p\right\} \vee$ $\left(\sum_{i=1}^{p}\left|a_{i}\right|^{4}\right)^{\frac{1}{4}}=\left(\sum_{i=1}^{p}\left|a_{i}\right|^{4}\right)^{\frac{1}{4}}=\|a\|_{4}$.

Lema 2.12 Se $T \in B(\mathrm{~W})$ e $\Lambda_{T}$ é a matriz associada a $T,\left(\phi(T)=\Lambda_{T}\right)$, então existe um único operador $T_{\Lambda} \in B\left(c_{0}\right)$, tal que $T_{\Lambda}(a)=\Lambda_{T} a, \forall a \in c_{00}$. Além disso, $\left\|T_{\Lambda}\right\| \leq\|T\|$.

Demonstração: Seja $T \in B(\mathbf{W}), T=0$, então $\lim _{n \rightarrow+\infty} T_{i j}\left(x_{n j}\right)-\lambda_{i j}\left(x_{n i}\right)=0$ é equivalente a $\lim _{n \rightarrow+\infty}-\lambda_{i j}\left(x_{n i}\right)=0$, isto é, $\lambda_{i j}=0$, logo $\Lambda_{T}=(0)_{i, j=1}^{\infty}$ e $T_{\Lambda}$ é o operador nulo de $c_{0}$.

Caso contrário podemos supor, sem perda de generalidade, que $\|T\|=1$.

Consideremos $L: c_{00} \rightarrow l_{\infty}$ definida por $L(a)=\Lambda_{T} a=\phi(T)(a), \quad \forall a \in c_{00}$.

Afirmamos que se $\|a\|_{\infty}=1$, então $\left\|\Lambda_{T} a\right\|_{\infty} \leq 1$. Provada esta afirmação, teremos mostrado que $L$ está bem definida, pois dado $a \in c_{00},\left\|\Lambda_{T} \frac{a}{\|a\|_{\infty}}\right\|_{\infty} \leq 1$ acarreta $\left\|\Lambda_{T} a\right\|_{\infty} \leq\|a\|_{\infty}$ e $\Lambda_{T} a \in l_{\infty}$, e também que a aplicação linear $L$ de $c_{00}$ 
para $l_{\infty}$ é limitada, com norma menor ou igual a 1 .

Suponhamos que a afirmação anterior seja falsa, logo existe $a \in c_{00}$, com $\|a\|_{\infty}=$ 1 e $\varepsilon>0$, tais que $\left\|\Lambda_{T} a\right\|_{\infty} \geq 1+3 \varepsilon$.

Seja $b=\Lambda_{T} a$, fixemos $i$, tal que $\left|b_{i}\right| \geq 1+2 \varepsilon$ (isto é possível, pois $\left\|\Lambda_{T} a\right\|_{\infty} \geq$ $1+3 \varepsilon)$

Pelo Lema 2.10, temos que $T\left(a \otimes x_{n}\right)-b \otimes x_{n}$ converge pontualmente a zero, isto é, $\lim _{n \rightarrow+\infty} P_{i}\left(T\left(a \otimes x_{n}\right)\right)-b_{i} x_{n i}=0$, para todo $i \in \mathbb{N}$.

Logo, para $i$ fixado anteriormente, existirá $n_{i}$ tal que se $n>n_{i}$, então $\| P_{i}(T(a \otimes$ $\left.\left.x_{n}\right)\right)-b_{i} x_{n i} \|<\frac{\varepsilon}{2}$.

Temos também $\left\|b_{i} x_{n i}\right\|=\left|b_{i}\right| \geq 1+2 \varepsilon, \forall n \in \mathbb{N}$. Como $\left\|b_{i} x_{n i}\right\|=\| P_{i}\left(T\left(a \otimes x_{n}\right)\right)+$ $b_{i} x_{n i}-P_{i}\left(T\left(a \otimes x_{n}\right)\right)\|\leq\| P_{i}\left(T\left(a \otimes x_{n}\right)\right)-b_{i} x_{n i}\|+\| P_{i}\left(T\left(a \otimes x_{n}\right)\right) \|$, podemos tomar $n_{0} \in N_{0}, n_{0}>n_{i}$ tal que $\left\|P_{i}\left(T\left(a \otimes x_{n_{0}}\right)\right)\right\| \geq\left\|b_{i} x_{n_{0} i}\right\|-\left\|P_{i}\left(T\left(a \otimes x_{n_{0}}\right)\right)-b_{i} x_{n_{0} i}\right\| \geq$ $1+2 \varepsilon-\frac{\varepsilon}{2} \geq 1+\varepsilon$.

Segue que $1+\varepsilon \leq \| P_{i}\left(T\left(a \otimes x_{n_{0}}\right)\|\leq\| T\left(a \otimes x_{n_{0}}\right) \|\right.$, mas $n_{0} \in N_{0}$, logo pelo Lema 2.11 item (a), $\left\|a \otimes x_{n_{0}}\right\|_{V}=\|a\|_{\infty}=1$;

Obtemos assim $1+\varepsilon \leq\left\|T\left(a \otimes x_{n_{0}}\right)\right\| \leq\left\|a \otimes x_{n_{0}}\right\|_{V}=1$, o que é uma contradição.

Assim, $L: c_{00} \rightarrow l_{\infty}$ é uma aplicação linear limitada com $\|L\| \leq 1$. Podemos estender $L$ a um operador único $T_{\Lambda}: c_{0} \rightarrow l_{\infty}, \operatorname{com} T_{\Lambda}(a)=L(a)=\Lambda_{T} a, \forall a \in c_{00}$ e $\left\|T_{\Lambda}\right\| \leq 1$. Mostramos, que se $\|T\|=1$, então $\left\|T_{\Lambda}\right\| \leq 1$, isto é, $\forall T \in B(\mathbf{W})$, $\left\|T_{\Lambda}\right\| \leq\|T\|$.

Vamos agora, utilizando argumentos semelhantes, mostrar que $T_{\Lambda}$ é um operador limitado de $c_{0}$ para $c_{0}$. 
Consideraremos para $a \in c_{00}$, com máx supp $(a)=p,\|a\|_{4}=\left(\sum_{i=1}^{p}\left|a_{i}\right|^{4}\right)^{\frac{1}{4}}$.

Dado $T \in B(\mathrm{~W})$, seja $L_{1}: c_{00} \rightarrow l_{4}$ definida por $L_{1}(a)=\Lambda_{T} a, \forall a \in c_{00}$. Afirmamos que se $\|a\|_{4}=1$, então $\left\|\Lambda_{T} a\right\|_{4} \leq 1$, onde denotando $b=\Lambda_{T} a,\left\|\Lambda_{T} a\right\|_{4}=$ $\left(\sum_{i=1}^{\infty}\left|b_{i}\right|^{4}\right)^{\frac{1}{4}}$.

Provada esta afirmação, teremos mostrado como no caso anterior que dado $a \in$ $c_{00},\left\|\Lambda_{T} \frac{a}{\|a\|_{4}}\right\|_{4} \leq 1$ acarreta $\left\|\Lambda_{T} a\right\|_{4} \leq\|a\|_{4}$, logo $\Lambda_{T} a \in l_{4}$ e $L_{1}$ estará bem definida e assim, mostraremos que se $a \in c_{00}$, então $\Lambda_{T} a \in c_{0}$.

Suponhamos novamente que a afirmação seja falsa, logo existem $a \in c_{00}$ com $\|a\|_{4}=1$ e $\varepsilon>0$ tais que $\left\|\Lambda_{T} a\right\|_{4}=\left(\sum_{i=1}^{\infty}\left|b_{i}\right|^{4}\right)^{\frac{1}{4}} \geq 1+3 \varepsilon$. Fixemos $M \in \mathbb{N}$ tal que $\left(\sum_{i=1}^{M}\left|b_{i}\right|^{4}\right)^{\frac{1}{4}} \geq 1+2 \varepsilon$ (isto é possível, pois $\left\|\Lambda_{T} a\right\|_{4} \geq 1+3 \varepsilon$ ).

Pelo Lema 2.10, temos que $T\left(a \otimes x_{n}\right)-b \otimes x_{n}$ converge pontualmente a zero, isto é, $\lim _{n \rightarrow+\infty} P_{i}\left(T\left(a \otimes x_{n}\right)\right)-b_{i} x_{n i}=0$.

Portanto existem $n_{1}, n_{2}, \ldots, n_{M}$ naturais, tais que se $n>\operatorname{máx}\left\{n_{1}, n_{2}, \ldots, n_{M}\right\}$, então $\left\|P_{i}\left(T\left(a \otimes x_{n}\right)\right)-b_{i} x_{n i}\right\| \leq \frac{\varepsilon}{2 i}, 1 \leq i \leq M$.

Segue que existe $n_{0} \in N_{4}, n_{0}>\operatorname{máx}\left\{n_{1}, \ldots, n_{M}\right\}$ tal que

$$
\left\|P_{i}\left(T\left(a \otimes x_{n_{0}}\right)\right)-b_{i} x_{n_{0} i}\right\| \leq \frac{\varepsilon}{2 i}, 1 \leq i \leq M .
$$

Temos também que para todo $\left.i, 1 \leq i \leq M, \mid x_{n_{0}}^{*}\left(P_{i}\left(T\left(a \otimes x_{n_{0}}\right)\right)\right)-b_{i} x_{n_{0} i}\right) \mid \leq$ $\left\|x_{n_{0}}^{*}\right\|\left\|P_{i}\left(T\left(a \otimes x_{n_{0}}\right)\right)-b_{i} x_{n_{0} i}\right\|=\left\|P_{i}\left(T\left(a \otimes x_{n_{0}}\right)\right)-b_{i} x_{n_{0} i}\right\|$.

Assim, de (2.1) teremos também:

$$
\left.\mid x_{n_{0}}^{*}\left(P_{i}\left(T\left(a \otimes x_{n_{0}}\right)\right)\right)-b_{i} x_{n_{0} i}\right) \mid \leq \frac{\varepsilon}{2 i}, \quad 1 \leq i \leq M .
$$


Além disso, da Observação 1.19, $\left\|T\left(a \otimes x_{n_{0}}\right)\right\| \geq\left\|\sum_{i=1}^{M} P_{i}\left(T\left(a \otimes x_{n_{0}}\right)\right)\right\|_{V}$.

Pela definição de norma em $\mathrm{V}$ e considerando $P_{i}\left(T\left(a \otimes x_{n_{0}}\right)\right)=z_{i} \in X_{i}$ e $\mathrm{w}_{j}=z_{2 j-1}+z_{2 j}$, teremos:

$$
\begin{aligned}
\left\|\sum_{i=1}^{M} P_{i}\left(T\left(a \otimes x_{n_{0}}\right)\right)\right\|_{V} \geq \sup \left\{\left(\sum_{j=1}^{\infty}\left|v_{n j}^{*}\left(\mathrm{w}_{j}\right)\right|^{4}+\left|w_{n j}^{*}\left(\mathrm{w}_{j}\right)\right|^{4}\right)^{\frac{1}{4}}: n \in N_{4}\right\} \\
\geq\left(\left|x_{n_{0}}^{*}\left(z_{1}\right)\right|^{4}+\left|x_{n_{0}}^{*}\left(z_{2}\right)\right|^{4}+\cdots+\left|x_{n_{0}}^{*}\left(z_{M}\right)\right|^{4}\right)^{\frac{1}{4}} \\
=\left(\sum_{i=1}^{M}\left|x_{n_{0}}^{*}\left(P_{i}\left(T\left(a \otimes x_{n_{0}}\right)\right)\right)\right|^{4}\right)^{\frac{1}{4}}
\end{aligned}
$$

Observemos também que $b_{i}=x_{n_{0}}^{*}\left(b_{i} x_{n_{0} i}\right)-x_{n_{0}}^{*}\left(P_{i}\left(T\left(a \otimes x_{n_{0}}\right)\right)\right)+x_{n_{0}}^{*}\left(P_{i}(T(a \otimes\right.$ $\left.x_{n_{0}}\right)$ )), logo pela desigualdade de Minkowsky [Ho, pág. 98], podemos escrever:

$$
\left.\left(\sum_{i=1}^{M}\left|x_{n_{0}}^{*}\left(P_{i}\left(T\left(a \otimes x_{n_{0}}\right)\right)\right)\right|^{4}\right)^{\frac{1}{4}} \geq\left(\sum_{i=1}^{M}\left|b_{i}\right|^{4}\right)^{\frac{1}{4}}-\left.\left(\sum_{i=1}^{M} \mid x_{n_{0}}^{*}\left(P_{i}\left(T\left(a \otimes x_{n_{0}}\right)\right)\right)-b_{i} x_{n_{0} i}\right)\right|^{4}\right)^{\frac{1}{4}}
$$

Assim, de (2.2), (2.3) e (2.4), obtemos $\left\|T\left(a \otimes x_{n_{0}}\right)\right\| \geq\left(\sum_{i=1}^{M}\left|x_{n_{0}}^{*}\left(P_{i}\left(T\left(a \otimes x_{n_{0}}\right)\right)\right)\right|^{4}\right)^{\frac{1}{4}} \geq$ $\left(\sum_{i=1}^{M}\left|b_{i}\right|^{4}\right)^{\frac{1}{4}}-\left(\sum_{i=1}^{M} \frac{\varepsilon^{4}}{\left(2^{4}\right)^{i}}\right)^{\frac{1}{4}} \geq\left(\sum_{i=1}^{M}\left|b_{i}\right|^{4}\right)^{\frac{1}{4}}-\varepsilon\left(\sum_{i=1}^{M} \frac{1}{\left(2^{4}\right)^{i}}\right)^{\frac{1}{4}} \geq\left(\sum_{i=1}^{M}\left|b_{i}\right|^{4}\right)^{\frac{1}{4}}-\varepsilon \geq$ $1+2 \varepsilon-\varepsilon=1+\varepsilon$. Mas, $n_{0} \in N_{4}$, logo pelo Lema 2.11 item (b), $\left\|a \otimes x_{n_{0}}\right\|_{V}=\|a\|_{4}=1$.

Obtemos então, $1+\varepsilon \leq\left\|T\left(a \otimes x_{n_{0}}\right)\right\| \leq\left\|a \otimes x_{n_{0}}\right\|_{V}=1$, o que é uma contradição.

Concluímos que dado $T \in B(\mathbf{W})$, podemos definir uma aplicação linear limitada $L: c_{00} \rightarrow c_{0}$ por $L(a)=\Lambda_{T} a, \forall a \in c_{00}$ e estender $L$ a um operador único limitado $T_{\Lambda}: c_{0} \rightarrow c_{0} \operatorname{com} T_{\Lambda}(a)=L(a)=\Lambda_{T} a, \forall a \in c_{00}$, além disso $\left\|T_{\Lambda}\right\| \leq\|T\|$.

Definição 2.13 A partir do lema anterior podemos definir uma aplicação $\Phi$ : $B(\mathbf{W}) \rightarrow B\left(c_{0}\right)$ por $\Phi(T)=T_{\Lambda}, \forall T \in B(\mathbf{W})$. 
Lema 2.14 (a) Sejam $a \in c_{00} e T \in B(\mathrm{~W})$. Suponhamos que exista $c \in c_{0}$ tal que $T\left(a \otimes x_{n}\right)-c \otimes x_{n}$ converge pontualmente a zero. Então $c=T_{\Lambda}(a)$.

(b) Sejam $c \in c_{0}, n \in N_{0} e S \in B(\mathrm{~W})$. Então a seqüência $S\left(c \otimes x_{n}\right)-$ $S_{\Lambda}(c) \otimes x_{n}$ converge pontualmente a zero.

Demonstração: (a) Seja $b=T_{\Lambda}(a)-c$. Consideremos a seqüência $b \otimes x_{n}$. Mostremos que $b \otimes x_{n}$ converge pontualmente a. zero.

De fato, $b \otimes x_{n}=\left(T_{\Lambda}(a)-c\right) \otimes x_{n}=T_{\Lambda}(a) \otimes x_{n}-c \otimes x_{n}=-T\left(a \otimes x_{n}\right)+T_{\Lambda}(a) \otimes$ $x_{n}+T\left(a \otimes x_{n}\right)-c \otimes x_{n}$.

Pelo Lema 2.10, $T\left(a \otimes x_{n}\right)-T_{\Lambda}(a) \otimes x_{n}$ converge pontualmente a zero e pela hipótese $T\left(a \otimes x_{n}\right)-c \otimes x_{n}$ converge pontualmente a zero, logo $b \otimes x_{n}$ converge pontualmente a zero.

Concluímos que $\lim _{n \rightarrow+\infty}\left(b \otimes x_{n}\right)_{i}=0$ para todo $i \in \mathbb{N}$, isto é, $\lim _{n \rightarrow+\infty}\left\|b_{i} x_{n i}\right\|=0$, conseqüentemente, $\left|b_{i}\right|=0$ e $b_{i}=0$. Assim, $b=0$ e $T_{\Lambda}(a)=c$.

(b) Suponhamos que $S=0, \operatorname{logo} S\left(c \otimes x_{n}\right)=0$. Temos também $S_{\Lambda}=0$, logo $S\left(c \otimes x_{n}\right)-S_{\Lambda}(c) \otimes x_{n}=0$ para todo $n \in \mathbb{N}$ e, portanto, converge pontualmente a zero.

Sejam então, $S \in B(\mathbf{W}), S \neq 0, c \in c_{0}, c=\sum_{i=1}^{\infty} c_{i} e_{i}$ e $\varepsilon>0$.

Existe $b \in c_{00}, b=\sum_{i=1}^{p} c_{i} e_{i}$ tal que $\|c-b\|_{\infty}<\frac{\varepsilon}{4\|S\|}$.

Consideremos para $n \in N_{0}$ a seqüência $S\left(c \otimes x_{n}-b \otimes x_{n}\right)=S\left((c-b) \otimes x_{n}\right)$.

Temos que $\left\|S\left((c-b) \otimes x_{n}\right)\right\| \leq\|S\|\left\|(c-b) \otimes x_{n}\right\|=\|S\|\|c-b\|_{\infty}<\|S\| \frac{\varepsilon}{4\|S\|}=\frac{\varepsilon}{4}$. Logo, para cada $i \in \mathbb{N}$,

$$
\left\|\left(S\left(c \otimes x_{n}\right)\right)_{i}-\left(S\left(b \otimes x_{n}\right)\right)_{i}\right\|=\left\|P_{i}\left(S\left((c-b) \otimes x_{n}\right)\right)\right\| \leq
$$




$$
\leq\left\|P_{i}\right\|\left\|S\left((c-b) \otimes x_{n}\right)\right\|<\frac{\varepsilon}{4}
$$

Analisemos agora, para $n \in N_{0}$, a seqüência $S_{\Lambda}(b) \otimes x_{n}-S_{\Lambda}(c) \otimes x_{n}=\left(S_{\Lambda}(b)-\right.$ $\left.S_{\Lambda}(c)\right) \otimes x_{n}=S_{\Lambda}(b-c) \otimes x_{n}$. Neste caso, temos $\left\|S_{\Lambda}(b-c) \otimes x_{n}\right\|=\left\|S_{\Lambda}(b-c)\right\|_{\infty} \leq$ $\left\|S_{\Lambda}\right\|\|b-c\|_{\infty} \leq\|S\| \frac{\varepsilon}{4\|S\|}=\frac{\varepsilon}{4}$. Assim, para cada $i \in \mathbb{N}$,

$$
\begin{gathered}
\left\|\left(S_{\Lambda}(b) \otimes x_{n}\right)_{i}-\left(S_{\Lambda}(c) \otimes x_{n}\right)_{i}\right\|=\left\|P_{i}\left(S_{\Lambda}(b-c) \otimes x_{n}\right)\right\| \\
\leq\left\|P_{i}\right\|\left\|S_{\Lambda}(b-c) \otimes x_{n}\right\|<\frac{\varepsilon}{4}
\end{gathered}
$$

Sabemos, pelo Lema 2.10, que $S\left(b \otimes x_{n}\right)-S_{\Lambda}(b) \otimes x_{n}$ converge pontualmente a. zero logo, para cada $i \in \mathbb{N}$, existe $n_{i} \in \mathbb{N}$ tal que se $n>n_{i}$, teremos

$$
\left\|\left(S\left(b \otimes x_{n}\right)\right)_{i}-\left(S_{\Lambda}(b) \otimes x_{n}\right)_{i}\right\|<\frac{\varepsilon}{2}
$$

Finalizando, podemos escrever: $\left\|\left(S\left(c \otimes x_{n}\right)\right)_{i}-\left(S_{\Lambda}(c) \otimes x_{n}\right)_{i}\right\| \leq \|\left(S\left(c \otimes x_{n}\right)_{i}-\right.$ $\left(S\left(b \otimes x_{n}\right)\right)_{i}\|+\|\left(S\left(b \otimes x_{n}\right)\right)_{i}-\left(S_{\Lambda}(b) \otimes x_{n}\right)_{i}\|+\|\left(S_{\Lambda}(b) \otimes x_{n}\right)_{i}-\left(S_{\Lambda}(c) \otimes x_{n}\right)_{i} \|$.

Assim, para cada $i \in \mathbb{N}$, dado $\varepsilon>0, \exists n_{i} \in N_{0}$ e $b \in c_{00}$ tais que, se $n>n_{i}$, utilizando (2.5), (2.6) e (2.7), obtemos $\left\|\left(S(c) \otimes x_{n}\right)_{i}-\left(S_{\Lambda}(c) \otimes x_{n}\right)_{i}\right\|<\frac{\varepsilon}{4}+\frac{\varepsilon}{2}+\frac{\varepsilon}{4}=$ $\varepsilon$.

Concluímos que para $n \in N_{0}$, a seqüência $S\left(c \otimes x_{n}\right)-S_{\Lambda}(c) \otimes x_{n}$ converge pontualmente a zero.

Observação 2.15 Podemos também concluir que para $n \in N_{0}, c \in c_{0}$ e $S \in B(\mathrm{~W})$, se existe $d \in c_{0}$ tal que a seqüência $S\left(c \otimes x_{n}\right)-d \otimes x_{n}$ converge pontualmente a zero, então $d=S_{\Lambda}(c)$. A demonstração é idêntica à do item (a) do lema anterior. Assim, esse resultado e o item (b) do lema anterior, caracterizam a imagem do operador $T_{\Lambda}$, isto é, se $T \in B(\mathbf{W}), \Phi(T)=T_{\Lambda}: c_{0} \rightarrow c_{0}$ é tal que $\operatorname{Im} T_{\Lambda}=\left\{d \in c_{0}\right.$ tal que $T\left(c \otimes x_{n}\right)-d \otimes x_{n}$ converge pontualmente a zero, para $c \in c_{0}$ e $\left.n \in N_{0}\right\}$. 
Lema 2.16 Se $T \in B(\mathbf{W})$ e $\left(\mathbf{z}_{n}\right)_{n=1}^{\infty} \in \mathbf{W}$ é uma seqüência limitada que converge pontualmente a zero, então $\left(T\left(\mathbf{z}_{n}\right)\right)_{n=1}^{\infty}$ converge pontualmente a zero.

Demonstração: Vamos supor que $\left(T\left(\mathbf{z}_{n}\right)\right)_{n=1}^{\infty}$ não converge pontualmente a zero, em conseqüência existem $\delta>0, i \in \mathbb{N}$ e uma subseqüência de $\left(T\left(\mathbf{z}_{n}\right)\right)_{n=1}^{\infty}$, que continuaremos denotando por $\left(T\left(\mathbf{z}_{n}\right)\right)_{n=1}^{\infty}$, tais que $\left\|P_{i}\left(T\left(\mathbf{z}_{n}\right)\right)\right\|>\delta$, para todo $n \in \mathbb{N}$.

Além disso, podemos a partir da hipótese, obter também uma subseqüência de $\left(\mathbf{z}_{n}\right)_{n=1}^{\infty},\left(\mathbf{z}_{n_{k}}\right)_{k=1}^{\infty}$ e uma seqüência de vetores $\left(\mathrm{v}_{k}\right)_{k=1}^{\infty}$ de $\mathrm{V}$, tais que $\left\|\mathrm{z}_{n_{k}}-\mathrm{v}_{k}\right\|<\frac{1}{2^{k}}$ e máx $\operatorname{supp}\left(\mathrm{v}_{k}\right)<\min \operatorname{supp}\left(\mathrm{v}_{k+1}\right)$, para todo $k \in \mathbb{N}$.

De fato, consideremos $\mathbf{z}_{n_{1}} \in \mathbf{W}, \mathbf{z}_{n_{1}}=\sum_{i=1}^{\infty} z_{n_{1} i}$. Existe $s_{1} \in \mathbb{N}$, tal que $\left\|\sum_{i=s_{1}+1}^{\infty} z_{n_{1} i}\right\|$ $<\frac{1}{2}$. Seja $\mathrm{v}_{1}=\sum_{i=1}^{s_{1}} z_{n_{1} i} \in \mathrm{V}$, portanto

$$
\left\|\mathbf{z}_{n_{1}}-\mathrm{v}_{1}\right\|=\left\|\sum_{i=s_{1}+1}^{\infty} z_{n_{1} i}\right\|<\frac{1}{2} .
$$

Agora, como $\left(\mathbf{z}_{n}\right)_{n=1}^{\infty}$ converge pontualmente a zero, existem $m_{1}, m_{2}, \ldots, m_{s_{1}+1}$ naturais, tais que $\left\|z_{m_{i} i}\right\|<\frac{1}{2^{3}} \frac{1}{2^{i}}$, para todo $i, 1 \leq i \leq s_{1}+1$.

Tomemos $n_{2} \in \mathbb{N}, n_{2}=\operatorname{máx}\left\{n_{1}, m_{1}, m_{2}, \ldots, m_{s_{1}+1}\right\} ;$ assim teremos $\left\|z_{n_{2} i}\right\|<$ $\frac{1}{2^{3}} \frac{1}{2^{i}}$, para todo $i, 1 \leq i \leq s_{1}+1$.

Consideremos então, $\mathbf{z}_{n_{2}} \in \mathbf{W}, \mathbf{z}_{n_{2}}=\sum_{i=1}^{\infty} z_{n_{2} i}$. Existe $s_{2}>s_{1}+2$, tal que $\left\|\sum_{i=s_{2}+1}^{\infty} z_{n_{2} i}\right\|<\frac{1}{2^{3}}$.

$$
\begin{gathered}
\text { Seja } \mathrm{v}_{2}=\sum_{i=s_{1}+2}^{s_{2}} z_{n_{2} i} \in \mathrm{V} \text {, portanto }\left\|\mathrm{z}_{n_{2}}-\mathrm{v}_{2}\right\|=\left\|\sum_{i=1}^{s_{1}+1} z_{n_{2} i}+\sum_{i=s_{2}+1}^{\infty} z_{n_{2} i}\right\| \leq \\
\left\|\sum_{i=1}^{s_{1}+1} z_{n_{2} i}\right\|+\left\|\sum_{i=s_{2}+1}^{\infty} z_{n_{2} i}\right\| \leq \sum_{i=1}^{s_{1}+1}\left\|z_{n_{2} i}\right\|+\left\|\sum_{i=s_{2}+1}^{\infty} z_{n_{2} i}\right\|<\frac{1}{2^{3}}\left(\sum_{i=1}^{s_{1}+1} \frac{1}{2^{i}}\right)+\frac{1}{2^{3}}<\frac{1}{2^{3}}+\frac{1}{2^{3}}=\frac{1}{2^{2}} .
\end{gathered}
$$

Temos também que máx supp $\left(\mathrm{v}_{1}\right)<\min \operatorname{supp}\left(\mathrm{v}_{2}\right)$. 
Vamos provar por indução que para todo $k \in \mathbb{N}$, existem $\mathrm{z}_{n_{k}} \in \mathrm{W}$ e $\mathrm{v}_{k} \in \mathrm{V}$,

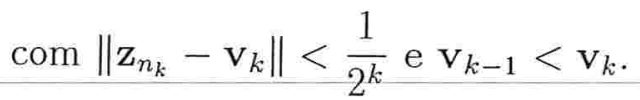

Suponhamos que para $k>2$ exista $\mathbf{z}_{n_{k}}=\sum_{i=1}^{\infty} z_{n_{k} i}$ e $\mathbf{v}_{k}=\sum_{i=s_{k-1}+2}^{s_{k}} z_{n_{k} i}$.

Como $\left(\mathbf{z}_{n}\right)_{n=1}^{\infty}$ converge pontualmente a zero, existem $m_{1}, m_{2}, \ldots, m_{s_{k}+1}$ naturais, tais que $\left\|z_{m_{i} i}\right\|<\frac{1}{2^{k+2}} \frac{1}{2^{i}}$, para todo $i, 1 \leq i \leq s_{k}+1$.

Tomemos $n_{k+1} \in \mathbb{N}, n_{k+1}=\operatorname{máx}\left\{n_{1}, n_{2}, \ldots, n_{k}, m_{1}, m_{2}, \ldots, m_{s_{k}+1}\right\}$; portanto $\left\|z_{n_{k+1} i}\right\|<\frac{1}{2^{k+2}} \frac{1}{2^{i}}$, para todo $i, 1 \leq i \leq s_{k}+1$.

Considerando $\mathbf{z}_{n_{k+1}} \in \mathrm{W}, \mathbf{z}_{n_{k+1}}=\sum_{i=1}^{\infty} z_{n_{k+1}}$, existe $s_{k+1}>s_{k}+2$, tal que $\left\|\sum_{i=s_{k+1}+1}^{\infty} z_{n_{k+1} i}\right\|<\frac{1}{2^{k+2}}$.

Seja então $\mathbf{v}_{k+1}=\sum_{i=s_{k}+2}^{s_{k+1}} z_{n_{k+1}}$. Portanto, obtemos $\left\|z_{n_{k+1}}-\mathrm{v}_{k+1}\right\| \leq \sum_{i=1}^{s_{k+1}}\left\|z_{n_{k+1}}\right\|$ $+\left\|\sum_{i=s_{k+1}+1}^{\infty} z_{n_{k+1} i}\right\|<\frac{1}{2^{k+2}}\left(\sum_{i=1}^{s_{k}+1} \frac{1}{2^{i}}\right)+\frac{1}{2^{k+2}}<\frac{1}{2^{k+1}}$ e também $\mathrm{v}_{k}<\mathrm{v}_{k+1}$.

Novamente, para facilitar a notação, denotemos a subseqüência $\left(\mathbf{z}_{n_{k}}\right)_{k=1}^{\infty}$ obtida, por $\left(\mathbf{z}_{n}\right)_{n=1}^{\infty}$; assim supondo falso o resultado do lema, obtivemos até aqui, uma sequência $\left(\mathbf{z}_{n}\right)_{n=1}^{\infty}$ de $\mathbf{W}$, uma seqüência $\left(\mathbf{v}_{n}\right)_{n=1}^{\infty}$ de $\mathrm{V}, i \in \mathbb{N}$ e $\delta>0$ tais que $\left\|\mathbf{z}_{n}-\mathrm{v}_{n}\right\|<\frac{1}{2^{n}}$ e $\left\|P_{i}\left(T\left(\mathbf{z}_{n}\right)\right)\right\|>\delta, \forall n \in \mathbb{N}$.

Olhemos agora para os vetores $P_{i}\left(T\left(\mathbf{z}_{n}\right)\right)$ pertencentes à cópia $X_{i}$. Seja $P_{i}\left(T\left(\mathbf{z}_{n}\right)\right)$ $=\sum_{k=1}^{\infty} \lambda_{k}^{n} e_{k}$ para todo $n \in \mathbb{N}$ (pois $\left(e_{k}\right)_{k=1}^{\infty}$ é base de $X$ ). Para estes vetores pode ocorrer uma das duas situações:

(1) $\lim _{n \rightarrow+\infty} \lambda_{k}^{n}=0, \forall k \in \mathbb{N}$, ou

(2) existem $k_{0} \in \mathbb{N}, \varepsilon>0$ e uma subseqüência $\left(\mathbf{z}_{n_{r}}\right)_{r=1}^{\infty}$ de $\left(\mathbf{z}_{n}\right)_{n=1}^{\infty}$, tais que $\left|\lambda_{k_{0}}^{n_{r}}\right|>\varepsilon, \forall r \in \mathbb{N}$. 
Comecemos pela (2). Neste caso, podemos supor sem perda de generalidade que $\lambda_{k_{0}}^{n_{r}}>\varepsilon, \forall r \in \mathbb{N}$ (pois haverá uma subseqüência de $\left(\mathbf{z}_{n_{r}}\right)_{r=1}^{\infty}$, que denotaremos também por $\left(\mathbf{z}_{n_{r}}\right)_{r=1}^{\infty}$ de termos positivos ou negativos que verificam a desigualdade (2)).

Logo, para todo $r \in \mathbb{N}$, teremos $\left\|T\left(\mathbf{z}_{n_{1}}+\mathrm{z}_{n_{2}}+\cdots+\mathbf{z}_{n_{r}}\right)\right\| \geq \| P_{i}\left(T\left(\mathbf{z}_{n_{1}}+\right.\right.$ $\left.\left.\cdots+\mathbf{z}_{n_{r}}\right)\right)\|=\| P_{i}\left(T\left(\mathbf{z}_{n_{1}}\right)\right)+\cdots+P_{i}\left(T\left(\mathbf{z}_{n_{r}}\right)\right)\|\geq\|\left(\lambda_{k_{0}}^{n_{1}}+\lambda_{k_{0}}^{n_{2}}+\cdots+\lambda_{k_{0}}^{n_{r}}\right) e_{k_{0}} \|=$ $\left|\lambda_{k_{0}}^{n_{1}}+\lambda_{k_{0}}^{n_{2}}+\cdots+\lambda_{k_{0}}^{n_{r}}\right|=\lambda_{k_{0}}^{n_{1}}+\cdots+\lambda_{k_{0}}^{n_{r}}>r \varepsilon$.

Assim, $\quad r \varepsilon \leq\left\|T\left(\sum_{i=1}^{r} \mathbf{z}_{n_{i}}\right)\right\| \leq\left\|T\left(\sum_{i=1}^{r} \mathbf{z}_{n_{i}}\right)-T\left(\sum_{i=1}^{r} \mathbf{v}_{n_{i}}\right)\right\|+\left\|T\left(\sum_{i=1}^{r} \mathbf{v}_{n_{i}}\right)\right\| \leq$ $\|T\|\left\|\sum_{i=1}^{r}\left(\mathbf{z}_{n_{i}}-\mathrm{v}_{n_{i}}\right)\right\|+\left\|T\left(\sum_{i=1}^{r} \mathrm{v}_{n_{i}}\right)\right\| \leq\|T\|\left(\sum_{i=1}^{r} \frac{1}{2^{n_{i}}}\right)+\|T\|\left\|\sum_{i=1}^{r} \mathrm{v}_{n_{i}}\right\|$.

Como $\left(\mathrm{v}_{n_{i}}\right)_{i=1}^{\infty}$ é uma seqüência de vetores sucessivos de $\mathrm{W}$, pelo Lema 2.1 , temos $\|T\|\left(\sum_{i=1}^{r} \frac{1}{2^{n_{i}}}\right)+\|T\|\left\|\sum_{i=1}^{r} \mathrm{v}_{n_{i}}\right\| \leq\|T\|+\|T\|\left(\sum_{i=1}^{r}\left\|\mathrm{v}_{n_{i}}\right\|^{2}\right)^{\frac{1}{2}} \leq\|T\|+\|T\|\left(\sum_{i=1}^{r}\left\|\mathrm{z}_{n_{i}}\right\|^{2}\right)^{\frac{1}{2}} \leq$ $\|T\|+\|T\|\left(r c^{2}\right)^{\frac{1}{2}}$, pois $\left(\mathbf{z}_{n}\right)_{n=1}^{\infty}$ é limitada, $\operatorname{logo}\left\|\mathbf{z}_{n_{i}}\right\| \leq c, c>0$, para todo $i \in \mathbb{N}$.

Concluímos então que para todo $r \in \mathbb{N}, r \varepsilon \leq\|T\|\left(1+r^{\frac{1}{2}} c\right)$, logo para $r>\frac{1}{c^{2}}$, isto é, $r^{\frac{1}{2}} c>1$, teremos

$$
r \varepsilon \leq\|T\| 2 r^{\frac{1}{2}} c \quad \text { e } \quad \varepsilon r^{\frac{1}{2}} \leq 2\|T\| c
$$

o que é um absurdo, pois $\lim _{r \rightarrow+\infty} \varepsilon r^{\frac{1}{2}}=+\infty$.

Analisemos então a situação (1). Suponhamos que $\lim _{n \rightarrow+\infty} \lambda_{k}^{n}=0$ para todo $k \in \mathbb{N}$. Neste caso, mostraremos que existirá $\varepsilon>0, \varepsilon=\frac{\delta}{4}$ e uma subseqüencia $\left(\mathbf{z}_{n_{r}}\right)_{r=1}^{\infty}$ de $\left(\mathbf{z}_{n}\right)_{n=1}^{\infty}$, tais que $\frac{\varepsilon r}{\log _{2}(r+1)} \leq\left\|T\left(\mathbf{z}_{n_{1}}+\mathbf{z}_{n_{2}}+\cdots+\mathbf{z}_{n_{r}}\right)\right\|$, para todo $r \in \mathbb{N}$. 
De fa.to, seja $P_{i}\left(T\left(\mathbf{z}_{n_{1}}\right)\right) \in X_{i}, \quad P_{i}\left(T\left(\mathbf{z}_{n_{1}}\right)\right)=\sum_{k=1}^{\infty} \lambda_{k}^{n_{1}} e_{k}$. Existe $s_{1} \in \mathbb{N}$, tal que $\left\|\sum_{k=s_{1}+1}^{\infty} \lambda_{k}^{n_{1}} e_{k}\right\|<\frac{\delta}{2^{3}}$, portanto $\left\|\sum_{k=1}^{s_{1}} \lambda_{k}^{n_{1}} e_{k}\right\|>\frac{\delta}{2}$ (pois $\left.\left\|P_{i}\left(T\left(\mathbf{z}_{n_{1}}\right)\right)\right\|>\delta\right)$.

Seja $u_{1} \in X_{i}, u_{1}=\sum_{k=1}^{s_{1}} \lambda_{k}^{n_{1}} e_{k}$, portanto $\left\|u_{1}\right\|>\frac{\delta}{2}$ e $\left\|P_{i}\left(T\left(\mathbf{z}_{n_{1}}\right)\right)-u_{1}\right\|<\frac{\delta}{2^{3}}$.

Como $\lim _{n \rightarrow+\infty} \lambda_{k}^{n}=0$, existem $m_{1}, m_{2}, \ldots, m_{s_{1}}$ tais que $\left|\lambda_{k}^{m_{k}}\right|<\frac{\delta}{2^{6}} \frac{1}{2^{k}}$, para todo $k, 1 \leq k \leq s_{1}$. Tomemos $n_{2}=\operatorname{máx}\left\{n_{1}, m_{1}, \ldots, m_{s_{1}}\right\}$ e obtemos $\left\|\lambda_{k}^{n_{2}} \epsilon_{k}\right\|<\frac{\delta}{2^{6}} \frac{1}{2^{k}}$, para todo $k, 1 \leq k \leq s_{1}$.

Consideremos $P_{i}\left(T\left(\mathbf{z}_{n_{2}}\right)\right)=\sum_{k=1}^{\infty} \lambda_{k}^{n_{2}} e_{k} \in X_{i}$. Existe $s_{2}>s_{1}$, tal que $\left\|\sum_{k=s_{2}+1}^{\infty} \lambda_{k}^{n_{2}} e_{k}\right\|$ $<\frac{\delta}{2^{6}}$.

Seja $u_{2} \in X_{i}, u_{2}=\sum_{k=s_{1}+1}^{s_{2}} \lambda_{k}^{n_{2}} e_{k}$, portanto $\left\|P_{i}\left(T\left(\mathbf{z}_{n_{2}}\right)\right)-u_{2}\right\|=\| \sum_{i=1}^{s_{1}} \lambda_{k}^{n_{2}} e_{k}$ $+\sum_{k=s_{2}+1}^{\infty} \lambda_{k}^{n_{2}} e_{k}\left\|\leq \sum_{k=1}^{s_{1}}\right\| \lambda_{k}^{n_{2}} e_{k}\|+\| \sum_{k=s_{2}+1}^{\infty} \lambda_{k}^{n_{2}} e_{k} \|<\frac{\delta}{2^{6}}\left(\sum_{k=1}^{s_{1}} \frac{1}{2^{k}}\right)+\frac{\delta}{2^{6}}<\frac{\delta}{2^{5}}$.

Temos também $\left\|u_{2}\right\|>\frac{\delta}{2}$ e min supp $\left(u_{1}\right)<\operatorname{máx} \operatorname{supp}\left(u_{2}\right)$ (suporte em relação à base $\left(e_{k}\right)_{k=1}^{\infty}$ de $\left.X_{i}\right)$.

Vamos provar por indução que para todo $r \in \mathbb{N}$, existem $P_{i}\left(T\left(\mathrm{z}_{n_{r}}\right)\right) \in X_{i}$ e $u_{r} \in X_{i}$, tais que $\left\|P_{i}\left(T\left(\mathrm{z}_{n_{r}}\right)\right)-u_{r}\right\|<\frac{\delta}{2^{2 r+2}},\left\|u_{r}\right\|>\frac{\delta}{2}$ e $u_{r-1}<u_{r}$.

Suponhamos que para $r>2$, tenhamos $P_{i}\left(T\left(\mathbf{z}_{n_{r}}\right)\right)=\sum_{k=1}^{\infty} \lambda_{k}^{n_{r}} e_{k}$ e $u_{r}=\sum_{k=s_{r-1}+1}^{s_{r}} \lambda_{k}^{n_{r}} e_{k}$.

Como $\lim _{n \rightarrow+\infty} \lambda_{k}^{n}=0$, existem $m_{1}, m_{2}, \ldots, m_{s_{k}}$ tais que $\left|\lambda_{k}^{m_{k}}\right|<\frac{\delta}{2^{2(r+1)+2}} \frac{1}{2^{k}}$, para todo $k, 1 \leq k \leq s_{r}$.

Tomemos $n_{r+1}=\operatorname{máx}\left\{n_{1}, n_{2}, \ldots, n_{k}, m_{1}, m_{2}, \ldots, m_{s_{k}}\right\}$ e obtemos $\left\|\lambda_{k}^{n_{r+1}} e_{k}\right\|<$ $\frac{\delta}{2^{2(r+1)+2}} \frac{1}{2^{k}}$, para todo $k, 1 \leq k \leq s_{r}$. 
Consideremos $P_{i}\left(T\left(\mathbf{z}_{n_{r+1}}\right)\right)=\sum_{k=1}^{\infty} \lambda_{k}^{n_{r+1}} e_{k} \in X_{i}$. Existe $s_{r+1}>s_{r}$, tal que $\left\|\sum_{k=s_{r+1}+1}^{\infty} \lambda_{k}^{n_{r+1}} e_{k}\right\|<\frac{\delta}{2^{2(r+1)+2}}$.

Seja $u_{r+1} \in X_{i}, u_{r+1}=\sum_{k=s_{r}+1}^{s_{r+1}} \lambda_{k}^{n_{r+1}} e_{k}$, portanto $\left\|P_{i}\left(T\left(\mathrm{z}_{n_{r+1}}\right)\right)-u_{r+1}\right\|=\| \sum_{k=1}^{s_{r}} \lambda_{k}^{n_{r+1}} e_{k}$ $+\sum_{k=s_{r+1}+1}^{\infty} \lambda_{k}^{n_{r+1}} e_{k}\left\|\leq \sum_{k=1}^{s_{r}}\right\| \lambda_{k}^{n_{r+1}} e_{k}\|+\| \sum_{k=s_{r+1}+1}^{\infty} \lambda_{k}^{n_{r+1}} e_{k} \|<\frac{\delta}{2^{2(r+1)+2}}\left(\sum_{k=1}^{s_{r}} \frac{1}{2^{k}}\right)$ $+\frac{\delta}{2^{2(r+1)+2}}<\frac{\delta}{2^{2(r+1)+1}}$.

Obtemos também $\left\|u_{r+1}\right\|>\frac{\delta}{2}$ e $u_{r}<u_{r+1}$.

Assim, para todo $r \in \mathbb{N}$, teremos $\left\|P_{i}\left(T\left(\mathbf{z}_{n_{1}}\right)\right)+P_{i}\left(T\left(\mathbf{z}_{n_{2}}\right)\right)+\cdots+P_{i}\left(T\left(\mathbf{z}_{n_{r}}\right)\right)\right\| \geq$ $\left\|u_{1}+u_{2}+\cdots+u_{r}\right\|-\left(\left\|u_{1}-P_{i}\left(T\left(\mathbf{z}_{n_{1}}\right)\right)\right\|+\cdots+\left\|u_{r}-P_{i}\left(T\left(\mathbf{z}_{n_{r}}\right)\right)\right\|\right)$.

Como $u_{1}<u_{2}<\cdots<u_{r}$, pelo Lema 1.1, temos que $\left\|u_{1}+u_{2}+\cdots+u_{r}\right\| \geq$ $r \frac{\delta}{2} \frac{1}{\log _{2}(r+1)}$.

Portanto, $\left\|T\left(\mathrm{z}_{n_{1}}+\overline{\mathrm{z}}_{n_{2}}+\cdots+\mathrm{z}_{n_{r}}\right)\right\| \geq\left\|P_{i}\left(T\left(\mathrm{z}_{n_{1}}\right)\right)+\cdots+P_{i}\left(T\left(\mathbf{z}_{n_{r}}\right)\right)\right\|>$ $r \frac{\delta}{2} \frac{1}{\log _{2}(r+1)}-\sum_{i=1}^{r} \frac{\delta}{2^{2 i+1}}>r \frac{\delta}{2} \frac{1}{\log _{2}(r+1)}-\frac{\delta}{4}=\frac{\delta}{4}\left(\frac{2 r}{\log _{2}(r+1)}-1\right) \geq$ $\frac{\delta}{4}\left(\frac{2 r}{\log _{2}(r+1)}-\frac{r}{\log _{2}(r+1)}\right)=\frac{\delta}{4}\left(\frac{r}{\log _{2}(r+1)}\right)$.

Concluímos como no caso anterior, utilizando o Lema 2.1, que para todo $r \in \mathbb{N}$, $r \frac{\delta}{4} \frac{1}{\log _{2}(r+1)} \leq\left\|T\left(\sum_{i=1}^{r} \mathbf{z}_{n_{i}}\right)\right\| \leq\|T\|\left(1+r^{\frac{1}{2}} c\right) \operatorname{logo}$, para $r>\frac{1}{c^{2}}$, isto é, $r^{\frac{1}{2}} c>1$, teremos

$$
r \frac{\delta}{4} \frac{1}{\log _{2}(r+1)} \leq\|T\| 2 r^{\frac{1}{2}} c \quad \text { e } \quad \frac{\delta}{4} \frac{r^{\frac{1}{2}}}{\log _{2}(r+1)} \leq 2\|T\| c
$$

o que é um absurdo, pois $\lim _{r \rightarrow+\infty} \frac{r^{\frac{1}{2}}}{\log _{2}(r+1)}=+\infty$.

Lema 2.17 Se $S$ e $T \in B(\mathrm{~W})$, então $\Phi(S T)=\Phi(S) \Phi(T)$, isto é, $(S T)_{\Lambda}=S_{\Lambda} T_{\Lambda}$. 
Demonstração: Dados $S, T \in B(\mathbf{W}), a \in c_{00}$, sejam $\Phi(T)=T_{\Lambda} \in B\left(c_{0}\right)$ e $\Phi(S)=$ $S_{\Lambda} \in B\left(c_{0}\right)$.

Pelo Lema 2.10, a seqüência $\left(\mathbf{z}_{n}\right)_{n=1}^{\infty}=T\left(a \otimes x_{n}\right)-T_{\Lambda}(a) \otimes x_{n}$ converge pontualmente a zero; ainda pelo Lema 2.12 , temos $\left\|\Lambda_{T} a\right\|_{\infty}=\left\|T_{\Lambda}(a)\right\|_{\infty} \leq\left\|T_{\Lambda}\right\|\|a\|_{\infty} \leq$ $\|T\|\|a\|_{\infty}$.

Se $n \in N_{0}$, então $z_{n} \in \mathrm{W}$; pois $T_{\Lambda}(a) \in c_{0}$ (pelo Lema 2.12) e $T_{\Lambda}(a) \otimes x_{n} \in \mathrm{W}$ (pelo Lema 2.11, item (a)). Temos também que $\left(\mathbf{z}_{n}\right)_{n \in N_{0}}$ é limitada, pois $\left\|\mathbf{z}_{n}\right\|=$ $\left\|T\left(a \otimes x_{n}\right)-T_{\Lambda}(a) \otimes x_{n}\right\| \leq\left\|T\left(a \otimes x_{n}\right)\right\|+\left\|T_{\Lambda}(a) \otimes x_{n}\right\| \leq\|T\|\left\|a \otimes x_{n}\right\|_{V}+\left\|T_{\Lambda}(a)\right\|_{\infty}$, mas, pela observação anterior, $\|T\|\left\|a \otimes x_{n}\right\|_{V}+\left\|T_{\Lambda}(a)\right\|_{\infty} \leq\|T\|\|a\|_{\infty}+\|T\|\|a\|_{\infty}=$ $2\|T\|\|a\|_{\infty}$.

Agora, consideremos

$$
\begin{aligned}
& (S T)\left(a \otimes x_{n}\right)=S\left(T\left(a \otimes x_{n}\right)\right)=S\left(T_{\Lambda}(a) \otimes x_{n}+\mathrm{z}_{n}\right)=S\left(T_{\Lambda}(a) \otimes x_{n}\right)-S_{\Lambda}\left(T_{\Lambda}(a)\right) \\
& \otimes x_{n}+S_{\Lambda}\left(T_{\Lambda}(a)\right) \otimes x_{n}+S\left(\mathrm{z}_{n}\right)=S_{\Lambda}\left(T_{\Lambda}(a)\right) \otimes x_{n}+\mathrm{w}_{n}+S\left(\mathrm{z}_{n}\right),
\end{aligned}
$$

onde $\mathbf{w}_{n}=S\left(T_{\Lambda}(a) \otimes x_{n}\right)-S_{\Lambda}\left(T_{\Lambda}(a)\right) \otimes x_{n}$.

A seqüência $\left(\mathbf{z}_{n}\right)_{n \in N_{0}}$ converge pontualmente a zero, logo pelo Lema 2.16 $\left(S\left(\mathbf{z}_{n}\right)\right)_{n \in N_{0}}$ converge pontualmente a zero.

Além disso, a seqüência $\left(\mathrm{w}_{n}\right)_{n \in N_{0}}$ converge pontualmente a zero, pelo Lema 2.14, item (b).

Assim, voltando à expressão $(2.8)$, temos $(S T)\left(a \otimes x_{n}\right)-S_{\Lambda}\left(T_{\Lambda}(a)\right) \otimes x_{n}=$ $\mathrm{w}_{n}+S\left(\mathbf{z}_{n}\right)$, que converge pontualmente a zero se $n \in N_{0}$. Logo, pelo Lema 2.14, item (a), temos $S_{\Lambda}\left(T_{\Lambda}(a)\right)=(S T)_{\Lambda}(a)$.

Concluímos que, para todo $a \in c_{00}, S_{\Lambda}\left(T_{\Lambda}(a)\right)=(S T)_{\Lambda}(a), \log \circ(S T)_{\Lambda}=S_{\Lambda} T_{\Lambda}$, 
isto é, $\Phi(S T)=\Phi(S) \Phi(T)$.

Lema $2.18 \Phi: B(\mathrm{~W}) \rightarrow B\left(c_{0}\right)$ é um homomorfismo de álgebra.

Demonstração: Para $a \in c_{00}, \Phi(T+S)(a)=\phi(T+S)(a)$ e pela Observação 2.6, $\phi(T+S)(a)=\phi(T)(a)+\phi(S)(a)=T_{\Lambda}(a)+S_{\Lambda}(a)=\Phi(T)(a)+\Phi(S)(a)$.

Assim, para todo $a \in c_{00}, \Phi(T+S)(a)=\Phi(T)(a)+\Phi(S)(a)$. Concluímos que $\Phi(T+S)=\Phi(T)+\Phi(S)$.

Analogamente, $\Phi(\gamma T)(a)=\phi(\gamma T)(a)$ e novamente pela Observação 2.6, $\phi(\gamma T)(a)$ $=\gamma \phi(T)(a)=\gamma T_{\Lambda}(a)=\gamma \Phi(T)(a)$, isto é, $\Phi(\gamma T)=\gamma \Phi(T), \forall \gamma \in \mathbb{R}$.

O lema anterior garante $\Phi(T S)=\Phi(T) \Phi(S)$. Pela Definição 0.30, $\Phi$ é um homomorfismo de álgebra.

A seguir, iremos enunciar e demonstrar dois resultados de álgebra linear, que serão utilizados na demonstração do Lema 2.21.

Lema 2.19 (a) Sejam $V$ um espaço vetorial e $S$ um subespaço de dimensão finita de $W=V \oplus V$, com a seguinte propriedade: se $(a, b) \in S$, então $(b, a) \in S,(a, 0) \in S$ $e(0, b) \in S$. Então, $\operatorname{dim} S$ é par.

(b) Sejam $V$ um espaço vetorial, $W=V \oplus V, P_{1}, P_{2}: W \rightarrow W$ projeções definidas por $P_{1}(a, b)=(a, 0)$ e $P_{2}(a, b)=(0, b), Z=P_{1}(Z) \oplus P_{2}(Z)$ um subespaço de $W$ de codimensão finita com a propriedade: $(a, 0) \in P_{1}(Z)$, se e somente se, $(0, a) \in P_{2}(Z)$. Então $P_{1}(Z)$ e $P_{2}(Z)$ têm codimensão finita em $V \oplus 0$ e $0 \oplus V$, respectivamente. Além disso, $Z$ tem codimensão par.

Demonstração: (a) Consideremos os seguintes subespaços de $S: S \cap(V \oplus 0)=S_{1}$ e $S \cap(0 \oplus V)=S_{2}$. Temos $S=S_{1} \oplus S_{2}$, pois $S_{1} \cap S_{2}=\{(0,0)\}$ e dado $(a, b) \in S$, $(a, b)=(a, 0)+(0, b) \operatorname{com}(a, 0) \in S_{1}$ e $(0, b) \in S_{2}$. 
Suponhamos inicialmente que $S_{1}=\{(0,0)\}$. Então, pela propriedade de $S$, $S_{2}=\{(0,0)\}$. Assim, $S=\{(0,0)\}$ e $\operatorname{dim} S=0$. Se $S_{1} \neq\{(0,0)\}$, tomemos uma base $\beta, \beta=\left\{\left(a_{1}, 0\right),\left(a_{2}, 0\right), \ldots,\left(a_{k}, 0\right)\right\}$ de $S_{1}$.

Pela propriedade do subespaço $S$, temos que $\beta^{\prime}=\left\{\left(0, a_{1}\right),\left(0, a_{2}\right), \ldots,\left(0, a_{k}\right)\right\}$ é um conjunto de vetores de $S_{2}$ e $\beta^{\prime}$ é base de $S_{2}$.

De fato, $\alpha_{1}\left(0, a_{1}\right)+\alpha_{2}\left(0, a_{2}\right)+\cdots+\alpha_{k}\left(0, a_{k}\right)=(0,0) \Leftrightarrow \alpha_{1} a_{1}+\alpha_{2} a_{2}+\cdots+\alpha_{k} a_{k}=0$, se e somente se, $\alpha_{1}=\alpha_{2}=\cdots=\alpha_{k}=0$, pois o conjunto $\beta$ é l.i. Portanto, $\beta^{\prime}$ é um conjunto l.i.

Agora, dado $(0, a) \in S_{2}$, consideremos o vetor $(a, 0) \in S_{1}$. Como $[\beta]=S_{1}$, existem $\alpha_{1}, \alpha_{2}, \ldots, \alpha_{k}$ tais que $(a, 0)=\alpha_{1}\left(a_{1}, 0\right)+\alpha_{2}\left(a_{2}, 0\right)+\cdots+\alpha_{k}\left(a_{k}, 0\right)$, isto é, $\alpha_{1} a_{1}+\alpha_{2} a_{2}+\cdots+\alpha_{k} a_{k}=a, \log 0(0, a)=\alpha_{1}\left(0, a_{1}\right)+\alpha_{2}\left(0, a_{2}\right)+\cdots+\alpha_{k}\left(0, a_{k}\right)$, portanto,

$$
\left[\beta^{\prime}\right]=S_{2}
$$

De (2.9) e (2.10), $\beta^{\prime}$ é base de $S_{2}$. Como $S=S_{1} \oplus S_{2}$ e $S$ tem dimensão finita, teremos $\operatorname{dim} S=\operatorname{dim} S_{1}+\operatorname{dim} S_{2}=2 k$.

(b) Se $W=V \oplus V$ tem dimensão finita, então $P_{1}(Z)$ e $P_{2}(Z)$ têm codimensão finita.

Segue que $V \oplus 0=P_{1}(Z) \oplus F_{1}$ e $0 \oplus V=P_{2}(Z) \oplus F_{2}$, com dimensão de $F_{1}$ e $F_{2}$ finita.

Logo, $\operatorname{dim} V \oplus 0=\operatorname{dim} P_{1}(Z)+\operatorname{dim} F_{1}=\operatorname{dim} P_{2}(Z)+\operatorname{dim} F_{2}=\operatorname{dim} 0 \oplus V$.

Mas, $P_{1}(Z) \sim P_{2}(Z)$, pelo isomorfismo $T: P_{1}(Z) \rightarrow P_{2}(Z)$ definido por $T(a, 0)=$ $(0, a), \forall(a, 0) \in P_{1}(Z), \operatorname{assim} \operatorname{dim} P_{2}(Z)=\operatorname{dim} P_{1}(Z)$ e $\operatorname{dim} F_{1}=\operatorname{dim} F_{2}$. 
Agora, $(a, b) \in V \oplus V$ se escreve de maneira única como soma de $\left(a_{1}, 0\right)+\left(f_{1}, 0\right) \in$ $P_{1}(Z)+F_{1}$ e $\left(0, a_{2}\right)+\left(0, f_{2}\right) \in P_{2}(Z)+F_{2}$, mas $\left(\left(a_{1}, 0\right)+\left(f_{1}, 0\right)\right)+\left(\left(0, a_{2}\right)+\right.$ $\left.\left(0, f_{2}\right)\right)=\left(\left(a_{1}, 0\right)+\left(0, a_{2}\right)\right)+\left(\left(f_{1}, 0\right)+\left(0, f_{2}\right)\right)$, onde $\left(a_{1}, 0\right)+\left(0, a_{2}\right) \in P_{1}(Z)+P_{2}(Z)$ e $\left(f_{1}, 0\right)+\left(0, f_{2}\right) \in F_{1}+F_{2}$.

Logo, podemos escrever $W=V \oplus V=\left(P_{1}(Z) \oplus P_{2}(Z)\right) \oplus\left(F_{1} \oplus F_{2}\right)=Z \oplus\left(F_{1} \oplus F_{2}\right)$. Concluímos que a codimensão de $Z$ é igual a $\operatorname{dim} F_{1} \oplus F_{2}=\operatorname{dim} F_{1}+\operatorname{dim} F_{2}=$ $2 \operatorname{dim} F_{1}$.

Analisemos agora o caso em que $\operatorname{dim} W$ é infinita. Inicialmente observemos que, dada uma base $\left\{\left(a_{i}, 0\right)_{i \in I}\right\}$ de $P_{1}(Z)$, devido à hipótese: $(a, 0) \in P_{1}(Z)$, se e somente se, $(0, a) \in P_{2}(Z)$, temos que $\left\{\left(0, a_{i}\right)_{i \in I}\right\}$ é base de $P_{2}(Z)$ e como $Z=P_{1}(Z) \oplus P_{2}(Z)$, teremos também que $B=\left\{\left(a_{i}, 0\right)_{i \in I} \cup\left(0, a_{i}\right)_{i \in I}\right\}$ é base de $Z$.

Suponhamos que $P_{1}(Z)$ tenha codimensão infinita, logo existe um conjunto infinito $\left\{\left(c_{j}, 0\right)_{j \in J}\right\}$ de vetores de $V \oplus 0$ tal que $A=\left\{\left(a_{i}, 0\right)_{i \in I} \cup\left(c_{j}, 0\right)_{j \in J}\right\}$ é um conjunto linearmente independente em $V \oplus 0$.

Pela hipótese, os vetores $\left(0, c_{j}\right)_{j \in J} \in P_{2}(Z)$ e considerando o conjunto $A^{\prime}=$ $\left\{\left(0, a_{i}\right)_{i \in I} \cup\left(0, c_{j}\right)_{j \in I}\right\}$, temos que $A^{\prime}$ é um conjunto de vetores linearmente independentes em $0 \oplus V$. Segue que o conjunto $D=\left\{\left(a_{i}, 0\right)_{i \in I} \cup\left(0, a_{i}\right)_{i \in I} \cup\left(c_{j}, 0\right)_{j \in J} \cup\right.$ $\left.\left(0, c_{j}\right)_{j \in J}\right\}$ é um conjunto de vetores linearmente independentes de $W$.

De fato, dada uma combinação linear desses vetores e igualando ao vetor nulo de $W$, teremos $\alpha_{1}\left(a_{1}, 0\right)+\cdots+\alpha_{p}\left(a_{p}, 0\right)+\beta_{1}\left(0, a_{1}^{\prime}\right)+\cdots+\beta_{q}\left(0, a_{q}^{\prime}\right)+\gamma_{1}\left(c_{1}, 0\right)+$ $\cdots+\gamma_{r}\left(c_{r}, 0\right)+\delta_{1}\left(0, c_{1}^{\prime}\right)+\cdots+\delta_{s}\left(0, c_{s}^{\prime}\right)=(0,0)$, se e somente se,

$$
\begin{aligned}
& \alpha_{1} a_{1}+\cdots+\alpha_{p} a_{p}+\gamma_{1} c_{1}+\cdots+\gamma_{r} c_{r}=0 \\
& \text { e } \quad \beta_{1} a_{1}^{\prime}+\cdots+\beta_{q} a_{q}^{\prime}+\delta_{1} c_{1}^{\prime}+\cdots+\delta_{s} c_{s}^{\prime}=0 .
\end{aligned}
$$


Como $A$ é um conjunto l.i., de (2.11) teremos $\alpha_{1}=\cdots=\alpha_{p}=\gamma_{1}=\cdots=\gamma_{r}=0$ e como $A^{\prime}$ é um conjunto 1.i., de (2.12), teremos $\beta_{1}=\cdots=\beta_{q}=\delta_{1}=\cdots=\delta_{s}=0$.

Concluímos que existe uma base $B$ de $Z$ e um conjunto infinito $C=\left\{\left(c_{j}, 0\right)_{j \in J} \cup\right.$ $\left.\left(0, c_{j}\right)_{j \in J}\right\}$ de vetores de $W$, tal que $B \cup C$ é um conjunto de vetores linearmente independentes de $W$, contrariando a hipótese de que $Z$ tem codimensão finita. Assim, a. codimensão de $P_{1}(Z)$ e de $P_{2}(Z)$ é finita.

Para finalizar, consideremos então que $V \oplus 0=P_{1}(Z) \oplus F_{1}$ tenha uma base $\left\{\left(a_{i}, 0\right)_{i \in I} \cup\left(c_{n}, 0\right)_{n=1}^{k}\right\}$, onde $\left(c_{n}, 0\right)_{n=1}^{k}$ é base de $F_{1}$ e portanto $\operatorname{dim} F_{1}=k$.

Como $\left\{\left(0, a_{i}\right)_{i \in I} \cup\left(0, c_{n}\right)_{n=1}^{k}\right\}$ é uma base de $0 \oplus V=P_{2}(Z) \oplus F_{2}$ e $\left(0, a_{i}\right)_{i \in I}$ é base de $P_{2}(Z)$, resulta que $\left(0, c_{n}\right)_{n=1}^{k}$ é base de $F_{2}$ e $\operatorname{assim} \operatorname{dim} F_{2}=k$.

Podemos escrever, como no caso finito, que $W=V \oplus V=\left(P_{1}(Z) \oplus P_{2}(Z)\right) \oplus\left(F_{1} \oplus\right.$ $\left.F_{2}\right)=Z \oplus\left(F_{1} \oplus F_{2}\right)$. Concluímos que a codimensão de $Z$ é igual a $\operatorname{dim}\left(F_{1} \oplus F_{2}\right)=$ $\operatorname{dim} F_{1}+\operatorname{dim} F_{2}=2 k$.

Lema 2.20 Sejam $V$ um espaço vetorial, $W=V \oplus V, A: V \rightarrow V$ um operador linear, $L: W \rightarrow W$ um operador linear definido por $L(a, b)=(A(a), A(b)), \forall(a, b) \in$ $W$. Se $\operatorname{dim} \operatorname{Ker} L<\infty$ e codimensão de $L(W)<\infty$, então a diferença entre $\operatorname{dim} \operatorname{Ker} L$ e a codimensão de $L(W)$ é par.

Demonstração: Se $(a, b) \in \operatorname{Ker} L$, então $(A(a), A(b))=(0,0), \operatorname{logo} A(a)=0$, $A(b)=0$ e como $A(0)=0$, teremos $(b, a) \in \operatorname{Ker} L,(a, 0) \in \operatorname{Ker} L$ e $(0, b) \in \operatorname{Ker} L$.

Concluímos pelo lema anterior item (a), que $\operatorname{dim} \operatorname{Ker} L$ é par.

Mostremos agora que codimensão de $L(W)$ é par. Preliminarmente, observemos que se $(A(a), A(b)) \in L(W)$, então existe $(a, b) \in W$, tal que $L(a, b)=(A(a), A(b))$, 
$\operatorname{logo} L(b, a)=(A(b), A(a)) \in L(W), L(a, 0)=(A(a), 0) \in L(W)$ e $L(0, b)=$ $(0, A(b)) \in L(W)$.

Considerando as projeções $P_{1}, P_{2}: W \rightarrow W$ definidas por $P_{1}(a, b)=(a, 0) \mathrm{e}$ $P_{2}(a, b)=(0, b)$, temos pela observação anterior que $P_{1}(L(W)) \subset L(W)$, pois se $(A(a), A(b)) \in W$, então $P_{1}(A(a), A(b))=(A(a), 0) \in L(W)$. Analogamente, temos $P_{2}(L(W)) \subset L(W)$.

Definindo $\left.P_{1}\right|_{L(W)}: L(W) \rightarrow L(W)$, temos que $\left.P_{1}\right|_{L(W)}$ é projeção, o mesmo ocorrendo com $\left.P_{2}\right|_{L(W)}: L(W) \rightarrow L(W)$.

Assim, podemos escrever que

$$
L(W)=P_{1}(L(W)) \oplus P_{2}(L(W))
$$

Temos também que

$$
(A(a), 0) \in P_{1}(L(W)) \Leftrightarrow(0, A(a)) \in P_{2}(L(W))
$$

De fato, se $(A(a), 0) \in P_{1}(L(W))$, então existe $(A(a), A(b)) \in L(W)$ tal que $P_{1}(A(a), A(b))=(A(a), 0) . \quad$ Como $(A(a), A(b)) \in L(W)$, então $(A(b), A(a)) \in$ $L(W)$, segue que $P_{2}(A(b), A(a))=(0, A(a))$ e $(0, A(a)) \in P_{2}(L(W))$. A recíproca se prova de forma análoga.

Finalizando, por hipótese temos que a codimensão de $L(W)$ é finita, além disso valendo (2.13) e (2.14), concluímos pelo lema anterior, item (b), que $L(W)$ tem codimensão par e assim, a diferença entre $\operatorname{dim} \operatorname{Ker} L$ e a codimensão de $L(W)$ é par.

Lema 2.21 Seja $T \in B(\mathrm{~W})$. Se $\Phi(T)=T_{\Lambda}$ é um operador de Fredholm com indice $n$, então $n$ é par. 
Demonstração: Dado $T \in B(\mathrm{~W})$, seja $\Phi(T)=T_{\Lambda}: c_{0} \rightarrow c_{0}$ o operador determinado no Lema 2.12.

Identifiquemos $c_{0}$ com $c_{0} \underset{\infty}{\oplus c_{0}}$; a partir da isometria: $\ell\left(a_{1}, b_{1}, a_{2}, b_{2}, \ldots\right) \mapsto\left(\left(a_{1}, a_{2}\right.\right.$, $\left.\ldots),\left(b_{1}, b_{2}, \ldots\right)\right)$.

Se $(a, b) \in c_{0} \bigoplus_{\infty} c_{0}$ e $z \in X$, definimos o produto $(a, b) \odot z$ por $(a, b) \odot z=$ $\ell^{-1}(a, b) \otimes z=\left(a_{1} z, b_{1} z, a_{2} z, b_{2} z, \ldots, a_{i} z, b_{i} z, \ldots\right)=\left(a_{1} z_{1}, b_{1} z_{2}, a_{2} z_{3}, b_{2} z_{4}, \ldots, a_{i} z_{2 i-1}\right.$, $\left.b_{i} z_{2 i}, \ldots\right)$ (veja Definição 2.7 ).

Seja $\tilde{T}_{\Lambda}: c_{0} \oplus_{\infty} c_{0} \rightarrow c_{0} \oplus_{\infty} c_{0}$ o operador limitado definido por $\tilde{T}_{\Lambda}(a, b)=\ell T_{\Lambda} \ell^{-1}(a, b)$, $\forall(a, b) \in c_{0} \oplus_{\infty} c_{0}$

Podemos para este operador escrever,

$$
\begin{aligned}
\tilde{T}_{\Lambda}(a, b) & =\tilde{T}_{\Lambda}((a, 0)+(0, b))=\tilde{T}_{\Lambda}(a, 0)+\tilde{T}_{\Lambda}(0, b) \\
& =\left(P_{1} \tilde{T}_{\Lambda} i_{1}(a), P_{2} \tilde{T}_{\Lambda} i_{1}(a)\right)+\left(P_{1} \tilde{T}_{\Lambda} i_{2}(b), P_{2} \tilde{T}_{\Lambda} i_{2}(b)\right)
\end{aligned}
$$

onde $P_{1}$ e $P_{2}$ são as projeções $P_{1}, P_{2}: c_{0} \oplus_{\infty} c_{0} \rightarrow c_{0} \bigoplus_{\infty} c_{0}$ definidas por $P_{1}(a, b)=(a, 0)$ e $P_{2}(a, b)=(0, b)$ respectivamente, e $i_{1}$ e $i_{2}$ são as inclusões $i_{1}, i_{2}: c_{0} \rightarrow c_{0} \underset{\infty}{\oplus} c_{0}$, definidas por $i_{1}(a)=(a, 0)$ e $i_{2}(b)=(0, b)$, respectivamente.

Assim, definindo os operadores limitados de $c_{0}, A=P_{1} \tilde{T}_{\Lambda} i_{1}, B=P_{1} \tilde{T}_{\Lambda} i_{2}, C=$ $P_{2} \tilde{T}_{\Lambda} i_{1}$ e $D=P_{2} \tilde{T}_{\Lambda} i_{2}$, podemos, da expressão (2.15), escrever:

$$
\begin{aligned}
\tilde{T}_{\Lambda}(a, b)= & (A(a), C(a))+(B(b), D(b))=(A(a)+B(b), C(a)+D(b)) \\
= & (A(a)+B(b), C(a)+D(b))-(0, A(b))+(0, A(b)) \\
= & (A(a), A(b))+(B(b), 0)+(0, C(a))-(0, A(b)-D(b)) \\
& \forall(a, b) \in c_{0} \bigoplus_{\infty} c_{0} .
\end{aligned}
$$

Agora, considerando os operadores de $c_{0} \oplus \oplus_{\infty} ; \tilde{T}_{\Lambda_{A}}, \tilde{T}_{\Lambda_{B}}, \tilde{T}_{\Lambda_{C}}$ e $\tilde{T}_{\Lambda_{A-D}}$ definidos 
por:

$$
\begin{aligned}
& \tilde{T}_{\Lambda_{A}}(a, b)=(A(a), A(b)), \quad \tilde{T}_{\Lambda_{B}}(a, b)=(B(b), 0), \\
& \tilde{T}_{\Lambda_{C}}(a, b)=(0, C(a)) \text { e } \tilde{T}_{\Lambda_{A-D}}(a, b)=(0, A(b)-D(b)) .
\end{aligned}
$$

Podemos finalmente escrever:

$$
\tilde{T}_{\Lambda}(a, b)=\tilde{T}_{\Lambda_{A}}(a, b)+\tilde{T}_{\Lambda_{S}}(a, b)
$$

onde

$$
\tilde{T}_{\Lambda_{S}}(a, b)=\tilde{T}_{\Lambda_{B}}(a, b)+\tilde{T}_{\Lambda_{C}}(a, b)-\tilde{T}_{\Lambda_{A-D}}(a, b) .
$$

Nosso objetivo é demonstrar que os operadores $B, C$ e $A-D$ são estritamente singulares e daí como $\tilde{T}_{\Lambda_{B}}=i_{1} B i_{2}^{-1} P_{2}$, pela. Proposição 0.25 item (b), $\tilde{T}_{\Lambda_{B}}$ é estritamente singular, o mesmo ocorrendo com $\tilde{T}_{\Lambda_{C}}=i_{2} C i_{1}^{-1} P_{1}$ e $\tilde{T}_{\Lambda_{A-D}}=i_{2}(A-D) i_{2}^{-1} P_{2}$.

Logo, pela Proposição 0.25 , item (a), teremos $\tilde{T}_{\Lambda_{S}}$ um operador estritamente singular.

Além disso, $\tilde{T}_{\Lambda}$ é um operador de Fredholm de índice $n$, já que por hipótese $T_{\Lambda}$ é um operador de Fredholm $\operatorname{com} i\left(T_{\Lambda}\right)=n$ e $i(\ell)=0=i\left(\ell^{-1}\right)$, pois $\ell$ é uma isometria sobrejetora.

Logo, pela Proposição 0.28 , teremos $i\left(\tilde{T}_{\Lambda}\right)=i\left(T_{\Lambda}\right)=n$. Mas, da expressão $(2.16)$ e pela Proposição $0.29, \tilde{T}_{\Lambda_{A}}=\tilde{T}_{\Lambda}-\tilde{T}_{\Lambda_{S}}$ é um operador de Fredhom com

$$
i\left(\tilde{T}_{\Lambda_{A}}\right)=i\left(\tilde{T}_{\Lambda}-\tilde{T}_{\Lambda_{S}}\right)=i\left(\tilde{T}_{\Lambda}\right)=n
$$

Finalizando a argumentação, como $\tilde{T}_{\Lambda_{A}}: c_{0} \bigoplus_{\infty} c_{0} \rightarrow c_{0} \oplus c_{0}$ é um operador de Fredholm definido por $\tilde{T}_{\Lambda_{A}}=(A(a), A(b))$, onde $A$ é um operador de $c_{0}$, temos pelo Lema 2.20 que a diferença entre $\operatorname{dim} \operatorname{Ker} \tilde{T}_{\Lambda_{A}}$ e a codimensão de $\tilde{T}_{\Lambda_{A}}\left(c_{0} \oplus c_{\infty}\right)$ é par, isto é, $i\left(\tilde{T}_{\Lambda_{A}}\right)$ é par. Logo, por (2.17), $n$ é par e assim, $i\left(T_{\Lambda}\right)=n$ é par. 
Comecemos então, supondo que o operador $B$ definido anteriormente não seja estritamente singular. Sendo assim, mostremos inicialmente que é possível determinar uma seqüência $\left(a_{n}\right)_{n=1}^{\infty}$ de vetores de $c_{0}$, normalizada, com $a_{n}<a_{n+1}$ e $\varepsilon>0$ tais que $\left\|B\left(a_{n}\right)\right\|_{\infty}>\varepsilon, \forall n \in \mathbb{N}$.

De fato, se $B$ não é estritamente singular, existe um subespaço fechado $A$ de $c_{0} \operatorname{com} \operatorname{dim} A=\infty$ e tal que $\left.B\right|_{A}$ é um isomorfismo sobre a imagem. Logo, existe $M>0$ tal que $\|B(c)\|_{\infty} \geq M\|c\|_{\infty}, \forall c \in A$.

Seja. $c_{1} \in A$, com $\left\|c_{1}\right\|_{\infty}=1$. Como $c_{1} \in c_{0}$, podemos escrever $c_{1}=\sum_{i=1}^{\infty} \alpha_{i}^{1} e_{i}=$ $\lim _{n \rightarrow+\infty} S_{n}$, onde $S_{n}=\sum_{i=1}^{n} \alpha_{i}^{1} e_{i}$ e também concluir que existe $n_{1} \in \mathbb{N}$ tal que máx $\left\{\left|\alpha_{i}\right|\right.$ : $i \in \mathbb{N}\}=\left|\alpha_{n_{1}}\right|=1$.

Consideremos então, $S_{n_{1}}=\sum_{i=1}^{n_{1}} \alpha_{i}^{1} e_{i}$. Logo, $\left\|S_{n_{1}}\right\|_{\infty}=1$. Além disso, $\left.B\right|_{A}$ é um operador limitado, logo $\lim _{n \rightarrow+\infty} B\left(S_{n}\right)=B\left(c_{1}\right)$, isto é, $\lim _{n \rightarrow+\infty}\left\|B\left(S_{n}\right)\right\|_{\infty}=\left\|B\left(c_{1}\right)\right\|_{\infty}$. Assim, existe $m_{1} \in \mathbb{N}$ tal que $\left\|B\left(S_{m_{1}}\right)\right\|_{\infty}>\frac{M}{2}$.

Podemos então, determinar $p_{1} \in \mathbb{N}, p_{1}=\operatorname{máx}\left\{n_{1}, m_{1}\right\}$, tal que $S_{p_{1}}=\sum_{i=1}^{p_{1}} \alpha_{i}^{1} e_{i}$ tem as seguintes propriedades: $\left\|S_{p_{1}}\right\|_{\infty}=1$ e $\left\|B\left(S_{p_{1}}\right)\right\|_{\infty}>\frac{M}{2}$. Tomemos $a_{1}=S_{p_{1}}$.

Agora, como $A$ é um subespaço de dimensão infinita de $c_{0}$ e $\left(e_{i}\right)_{i=1}^{\infty}$ é base de Schauder de $c_{0}$, pela Observação 0.11 , existe $c_{2} \in A$ da forma $c_{2}=\sum_{i=p_{1}+1}^{\infty} \alpha_{i}^{2} e_{i} \mathrm{e}$ $\left\|c_{2}\right\|_{\infty}=1$

Então, utilizando o raciocínio anterior, podemos encontrar $p_{2} \in \mathbb{N}\left(p_{2} \geq p_{1}+1\right)$ tal que $\left\|S_{p_{2}}\right\|_{\infty}=1$, onde $S_{p_{2}}=\sum_{i=p_{1}+1}^{p_{2}} \alpha_{i}^{2} \epsilon_{i}$ e $\left\|B\left(S_{p_{2}}\right)\right\|_{\infty}>\frac{M}{2}$. Ponhamos $a_{2}=S_{p_{2}}$.

Continuando desta forma, obtemos uma seqüência $\left(a_{n}\right)_{n=1}^{\infty}$ de vetores de $c_{0}$, com $a_{n}=S_{p_{n}}=\sum_{i=p_{n-1}+1}^{p_{n}} \alpha_{i}^{n} e_{i}$ (considerando $\left.p_{0}=0\right)$, tal que $\left\|a_{n}\right\|_{\infty}=1,\left\|B\left(a_{n}\right)\right\|_{\infty}>$ 
$\frac{M}{2}=\varepsilon$ e como $p_{n} \geq p_{n-1}+1>p_{n-1}$, temos máx $\operatorname{supp}\left(S_{p_{n}}\right)<\min \operatorname{supp}\left(S_{p_{n+1}}\right)$, isto é, $a_{n}<a_{n+1}, \forall n \in \mathbb{N}$.

Mostremos agora que é possível também determinar uma subseqüência $\left(a_{n_{p}}\right)_{p=1}^{\infty}$ de $\left(a_{n}\right)_{n=1}^{\infty}$, uma seqüência crescente $\left(j_{i}\right)_{i=1}^{\infty}, j_{i} \in \mathbb{N}$ e $\varepsilon>0$, tais que o módulo da. coordenada $j_{i}$ do vetor $B\left(a_{n_{i}}\right)$ é maior do que $\varepsilon$ e a soma do módulo da coordenada. $j_{i}, \operatorname{dos}\left(B\left(a_{n_{p}}\right)\right)$ vetores com $i \neq p$, é menor do que $\frac{\varepsilon}{2}$.

Para isso, observemos inicialmente que $a_{n} \stackrel{w}{\longrightarrow} 0$; pois $\left(a_{n}\right)_{n=1}^{\infty}$ é uma base de bloco de $\left(e_{n}\right)_{n=1}^{\infty}$ normalizada em $c_{0}$. Logo, pela Proposição $0.9,\left(a_{n}\right)_{n=1}^{\infty} \sim\left(e_{n}\right)_{n=1}^{\infty}$ e como $e_{n} \stackrel{w}{\longrightarrow} 0$, então $a_{n} \stackrel{w}{\longrightarrow} 0$.

De $a_{n} \stackrel{w}{\longrightarrow} 0$, temos $B\left(a_{n}\right) \stackrel{w}{\longrightarrow} 0$. Considerando a seqüência $\left(e_{j}^{*}\right)_{j=1}^{\infty}$ de funcionais biortogonais associados à base $\left(e_{i}\right)_{i=1}^{\infty}$ e $B\left(a_{n}\right)=\sum_{i=1}^{\infty} \gamma_{i}^{n} e_{i}$, temos que para todo $j \in \mathbb{N}$, $\lim _{n \rightarrow+\infty} e_{j}^{*}\left(B\left(a_{n}\right)\right)=\lim _{n \rightarrow+\infty} e_{j}^{*}\left(\sum_{i=1}^{\infty} \gamma_{i}^{n} e_{i}\right)=\lim _{n \rightarrow+\infty} \gamma_{j}^{n}=0$.

Vamos escrever, para facilitar a notação, $\gamma_{j}^{n}=\left(B\left(a_{n}\right)\right)_{j}$, a $j$-ésima coordenada do vetor $B\left(a_{n}\right) \in c_{0}$. Assim, $\lim _{n \rightarrow+\infty}\left(B\left(a_{n}\right)\right)_{j}=0, \forall j \in \mathbb{N}$.

Sejam $\varepsilon=\frac{M}{2}$ e $B\left(a_{1}\right)=B\left(a_{n_{1}}\right)$. Como $B\left(a_{n_{1}}\right) \in c_{0}$, existe $m_{1} \in \mathbb{N}$ tal que $\left|B\left(a_{n_{1}}\right)_{j}\right|<\frac{\varepsilon}{4}$ para $j \geq m_{1}$; além disso, $\left\|B\left(a_{n_{1}}\right)\right\|_{\infty}>\varepsilon$. Logo, existe $j_{1} \in \mathbb{N}$, $1 \leq j_{1}<m_{1}$, tal que $\left|B\left(a_{n_{1}}\right)_{j_{1}}\right|>\varepsilon$

Mas, $\lim _{n \rightarrow+\infty}\left(B\left(a_{n}\right)\right)_{j}=0$. Assim, dado $\frac{\varepsilon}{4}$, existe $n_{2} \in \mathbb{N}, n_{2}>n_{1}$ e $a_{n_{2}} \in c_{0}$ tais que $\left|\left(B\left(a_{n_{2}}\right)\right)_{j}\right|<\frac{\varepsilon}{4}$, para $1 \leq j \leq m_{1}$. Conseqüentemente, $\left|B\left(a_{n_{2}}\right)_{j_{1}}\right|<\frac{\varepsilon}{4}$.

Como $\left\|B\left(a_{n_{2}}\right)\right\|_{\infty}>\varepsilon$, existe $j_{2} \in \mathbb{N}, j_{2}>m_{1}>j_{1}$ tal que $\left|\left(B\left(a_{n_{2}}\right)\right)_{j_{2}}\right|>\varepsilon$. Teremos também $\mid\left(B\left(a_{n_{1}}\right)_{j_{2}} \mid<\frac{\varepsilon}{4}\right.$.

Agora, $B\left(a_{n_{2}}\right) \in c_{0}$. Logo, existe $m_{2} \in \mathbb{N}, m_{2}>j_{2}>m_{1}>j_{1}$, tal que se $j \geq m_{2}$, 
$\left|\left(B\left(a_{n_{2}}\right)\right)_{j}\right|<\frac{\varepsilon}{8}$

Como novamente $\lim _{n \rightarrow+\infty}\left(B\left(a_{n}\right)\right)_{j}=0$, dado $\frac{\varepsilon}{8}$ existe $n_{3} \in \mathbb{N}, n_{3}>n_{2}>n_{1}$ e $a_{n_{3}} \in c_{0}$, tais que $\left|\left(B\left(a_{n_{3}}\right)\right)_{j}\right|<\frac{\varepsilon}{8}$ se $1 \leq j \leq m_{2}$. Conseqüentemente, $\left|\left(B\left(a_{n_{3}}\right)\right)_{j_{1}}\right|<$ $\frac{\varepsilon}{8}$ e $\left|\left(B\left(a_{n_{3}}\right)\right)_{j_{2}}\right|<\frac{\varepsilon}{8}$. Mas $\left\|B\left(a_{n_{3}}\right)\right\|_{\infty}>\varepsilon$, logo, existe $j_{3} \in \mathbb{N}$, com $j_{3}>m_{2}>j_{2}>$ $m_{1}>j_{1}$, tal que $\left|\left(B\left(a_{n_{3}}\right)\right)_{j_{3}}\right|>\varepsilon$, teremos também, $\left|\left(B\left(a_{n_{1}}\right)\right)_{j_{3}}\right|<\frac{\varepsilon}{4} \mathrm{e}\left|\left(B\left(a_{n_{2}}\right)\right)_{j_{3}}\right|<$ $\frac{\varepsilon}{8}$.

Continuando este raciocínio, será possível determinar para todo $p \in \mathbb{N}, n_{p}$ com $n_{p+1}>n_{p}$ e conseqüentemente obter uma subseqüência $\left(a_{n_{p}}\right)_{p=1}^{\infty}$ de $\left(a_{n}\right)_{n=1}^{\infty}$ e também uma seqüência crescente $\left(j_{i}\right)_{i=1}^{\infty}$ de naturais, tais que $\left|\left(B\left(a_{n_{p}}\right)\right)_{j_{i}}\right|>\varepsilon$ se $p=i$ e $\sum_{p \neq i} \mid\left(B\left(a_{n_{p}}\right)_{j_{i}} \mid<\sum_{k=1}^{\infty} \frac{\varepsilon}{2^{2 k}}<\frac{\varepsilon}{2}\right.$.

A seguir, para facilitar a notação, denotaremos a seqüência $\left(a_{n_{p}}\right)_{p=1}^{\infty}$ simplesmente por $\left(a_{n}\right)_{n=1}^{\infty}$. Logo, obtemos $a_{n}<a_{n+1},\left\|a_{n}\right\|=1, \forall n \in \mathbb{N},\left|\left(B\left(a_{i}\right)\right)_{j_{i}}\right|>$ $\varepsilon$ e $\sum_{n \neq i}\left|\left(B\left(a_{n}\right)\right)_{j_{i}}\right|<\frac{\varepsilon}{2}$.

O próximo passo é mostrar que $\lim _{k \rightarrow+\infty} T\left(\left(0, a_{n}\right) \odot x_{k}\right)-\tilde{T}_{\Lambda}\left(0, a_{n}\right) \odot x_{k}=0$ pontualmente para todo $n \in \mathbb{N}$.

Pela definição dada no início do lema, para cada $n \in \mathbb{N},\left(0, a_{n}\right) \odot x_{k}=\ell^{-1}\left(0, a_{n}\right) \otimes$ $x_{k}=\left(0, a_{n 1}, 0, a_{n 2}, \ldots, 0, a_{n s_{n}}, 0, \ldots\right) \otimes x_{k}$, onde $s_{n}=\operatorname{máx} \operatorname{supp}\left(a_{n}\right)$.

Assim, podemos escrever para todo $n \in \mathbb{N},\left(0, a_{n}\right) \odot x_{k}=a_{n}^{p} \otimes x_{k}$, onde $a_{n}^{p}=$ $\left(0, a_{n 1}, 0, a_{n 2}, 0, \ldots, 0, a_{n s_{n}}, 0, \ldots\right) \in c_{00}$. Agora, $\tilde{T}_{\Lambda}\left(0, a_{n}\right)=\ell T_{\Lambda} \ell^{-1}\left(0, a_{n}\right)=\ell T_{\Lambda}\left(a_{n}^{p}\right)$

Por definição, $\tilde{T}_{\Lambda}\left(0, a_{n}\right) \odot x_{k}=\ell^{-1}\left(\tilde{T}_{\Lambda}\left(0, a_{n}\right)\right) \otimes x_{k}=\ell^{-1}\left(\ell T_{\Lambda}\left(a_{n}^{p}\right)\right) \otimes x_{k}=T_{\Lambda}\left(a_{n}^{p}\right) \otimes$ $x_{k}$.

Segue que $\lim _{k \rightarrow+\infty}\left(T\left(\left(0, a_{n}\right) \odot x_{k}\right)\right)_{i}-\left(\tilde{T}_{\Lambda}\left(0, a_{n}\right) \odot x_{k}\right)_{i}=\lim _{k \rightarrow+\infty}\left(T\left(a_{n}^{p} \otimes x_{k}\right)\right)_{i}-$ 
$\left(T_{\Lambda}\left(a_{n}^{p}\right) \otimes x_{k}\right)_{i}$

Mas como $a_{n}^{p} \in c_{00}$, pelo Lema 2.10 teremos $\lim _{k \rightarrow+\infty}\left(T\left(a_{n}^{p} \otimes x_{k}\right)\right)_{i}-\left(T_{\Lambda}\left(a_{n}^{p}\right) \otimes x_{k}\right)_{i}=$ 0 , para todo $n \in \mathbb{N}$.

Notemos que para todo $i, j, k$ e $n \in \mathbb{N}$ temos $v_{i j}^{*}\left(\left(0, a_{n}\right) \odot x_{k}\right)=0$. De fato, para cada $n \in \mathbb{N},\left(0, a_{n}\right) \odot x_{k}=a_{n}^{p} \otimes x_{k}=0+a_{n 1} x_{k 2}+0+a_{n 2} x_{k 4}+\cdots+0+a_{n s_{n}} x_{k 2 s_{n}}$, onde máx $\operatorname{supp}\left(a_{n}\right)=s_{n}$.

Logo, a projeção $P_{j}$ do vetor $\left(0, a_{n}\right) \odot x_{k}$ em $\mathcal{W}_{j}$ tem a primeira componente em $X_{2 j-1}$ igual a zero, isto é, $P_{j}\left(\left(0, a_{n}\right) \odot x_{k}\right)=0+a_{n j} x_{k 2 j} \in \mathcal{W}_{j}$. Assim, $v_{i j}^{*}\left(\left(0, a_{n}\right) \odot\right.$ $\left.x_{k}\right)=\left(x_{i}^{*}, 0\right)\left(0, a_{n j} x_{k 2 j}\right)=x_{i}^{*}(0)-0 a_{n j} x_{k 2 j}=0$.

Se $k \in N_{2}$ e $i \in N_{1} \cup N_{3} \cup N_{4}$, então $i \neq k$, logo o mesmo ocorre com os funcionais $u_{i j}^{*}$ e $w_{i j}^{*}$, isto é, $u_{i j}^{*}\left(\left(0, a_{n}\right) \odot x_{k}\right)=\left(x_{i}^{*},-x_{i}^{*}\right)\left(0, a_{n j} x_{k 2 j}\right)=x_{i}^{*}(0)-x_{i}^{*}\left(a_{n j} x_{k 2 j}\right)=$ $-a_{n j} x_{i}^{*}\left(x_{k 2 j}\right)=-a_{n j} 0=0$ e $w_{i j}^{*}\left(\left(0, a_{n}\right) \odot x_{k}\right)=\left(0, x_{i}^{*}\right)\left(0, a_{n j} x_{k 2 j}\right)=x_{i}^{*}\left(a_{n j} x_{k 2 j}\right)=$ $a_{n j} x_{i}^{*}\left(x_{k 2 j}\right)=a_{n j} 0=0$.

Dado $N \in \mathbb{N}$, consideremos o vetor de $\mathbf{V}, \sum_{n=1}^{N}\left(0, a_{n}\right) \odot x_{k}=\sum_{n=1}^{N} a_{n}^{p} \otimes x_{k}=$ $0+a_{11} x_{k 2}+0+a_{12} x_{k 4}+\cdots+0+a_{1 s_{1}} x_{k 2 s_{1}}+0+a_{2 s_{1}+1} x_{k 2\left(s_{1}+1\right)}+\cdots+0+a_{2 s_{2}} x_{k 2 s_{2}}+$ $\cdots+0+a_{N s_{N-1}+1} x_{k 2\left(s_{N-1}+1\right)}+\cdots+0+a_{N s_{N}} x_{k 2 s_{N}}$ e $k \in N_{2}$.

Pela definição de norma e das observações anteriores obtemos, para todo $N \in \mathbb{N}$, $\left\|\sum_{n=1}^{N}\left(0, a_{n}\right) \odot x_{k}\right\|_{V}=\operatorname{máx}\left\{\left\|w_{j}^{n}\right\|: 1 \leq n \leq N\right.$ e $\left.1 \leq j \leq s_{n}\right\}$, onde $w_{j}=$ $\left(0, a_{n j} x_{k 2 j}\right)$; e a norma é a de um vetor do espaço $\mathcal{W}_{j}=X_{2 j-1} \oplus X_{2 j}$.

Segue que, $\operatorname{máx}\left\{\left\|w_{j}^{n}\right\|: 1 \leq n \leq N\right.$ e $\left.1 \leq j \leq s_{n}\right\}=\operatorname{máx}_{1 \leq n \leq N}\left\{\operatorname{máx}\left\|a_{n j} x_{k 2 j}\right\|\right.$ $\left.: 1 \leq j \leq s_{n}\right\}=\operatorname{máx}_{1 \leq n \leq N}\left\{\operatorname{máx}\left|a_{n j}\right|: 1 \leq j \leq s_{n}\right\}=\operatorname{máx}\left\{\left\|a_{n}^{p}\right\|_{\infty}: 1 \leq n \leq N\right\}=$ máx $\left\{\left\|a_{n}\right\|_{\infty}: 1 \leq n \leq N\right\}$. 
Mas, $a_{1}<a_{2}<\cdots<a_{N}$ e $\left\|a_{n}\right\|_{\infty}=1$, para $1 \leq n \leq N$. Logo, $\operatorname{máx}\left\{\left\|a_{n}\right\|_{\infty}: 1 \leq n \leq N\right\}=\left\|a_{1}+a_{2}+\cdots+a_{N}\right\|_{\infty}=\left\|\sum_{n=1}^{N} a_{n}\right\|_{\infty}=1$.

Façamos agora uma estimativa da norma do vetor $T\left(\sum_{n=1}^{N}\left(0, a_{n}\right) \odot x_{k}\right)$.

Em primeiro lugar, observemos que pela definição dada dos operadores $B$ e $D$, temos que $\tilde{T}_{\Lambda}\left(0, a_{n}\right)=\left(B\left(a_{n}\right), D\left(a_{n}\right)\right)$.

Concluímos anteriormente que para todo $n \in \mathbb{N}, T\left(\left(0, a_{n}\right) \odot x_{k}\right)-\left(B\left(a_{n}\right), D\left(a_{n}\right)\right)$ $\odot x_{k}$ converge pontualmente a zero se $k \rightarrow+\infty$. Fixemos $i \in \mathbb{N}$. Logo, dado $\varepsilon>0$, para $1 \leq n \leq N$, existirá $k_{n} \in \mathbb{N}$ tal que, se $k>k_{n}$, então $\|\left(T\left(\left(0, a_{n}\right) \odot x_{k}\right)\right)_{i}-$ $\left(\left(B\left(a_{n}\right), D\left(a_{n}\right)\right) \odot x_{k}\right)_{i} \|<\frac{\varepsilon}{N}$, onde a norma é a de um vetor pertencente a $X_{i}$.

Tomemos $k_{0}=\operatorname{máx}\left\{k_{n}: 1 \leq n \leq N\right\}$, logo se $k>k_{0}$ teremos $\| \sum_{n=1}^{N}\left(T\left(\left(0, a_{n}\right) \odot x_{k}\right)\right)_{i}$ $-\sum_{n=1}^{N}\left(\left(B\left(a_{n}\right), D\left(a_{n}\right)\right) \odot x_{k}\right)_{i}\left\|\leq \sum_{n=1}^{N}\right\|\left(T\left(\left(0, a_{n}\right) \odot x_{k}\right)\right)_{i}-\left(\left(B\left(a_{n}\right), D\left(a_{n}\right)\right) \odot x_{k}\right)_{i} \|$ $\leq \frac{\varepsilon}{N} N=\varepsilon$.

Assim, para todo $N \in \mathbb{N}$, a seqüência

$T\left(\sum_{n=1}^{N}\left(0, a_{n}\right) \odot x_{k}\right)-\sum_{n=1}^{N}\left(B\left(a_{n}\right), D\left(a_{n}\right)\right) \odot x_{k}$ converge pontualmente

a. zero, se $k \rightarrow+\infty$

Segue que, dados $N \in \mathbb{N}, \varepsilon>0$ e $j \in \mathbb{N}$, podemos determinar por (2.18), $k_{j} \in \mathbb{N}$, tal que se $k>k_{j}$,

$$
\begin{aligned}
& \left\|\left[T\left(\sum_{n=1}^{N}\left(0, a_{n}\right) \odot x_{k}\right)-\sum_{n=1}^{N}\left(B\left(a_{n}\right), D\left(a_{n}\right)\right) \odot x_{k}\right]_{j}\right\|_{V} \\
\leq & 2 \operatorname{máx}\left\{\left\|\left(T\left(\sum_{n=1}^{N}\left(0, a_{n}\right) \odot x_{k}\right)\right)_{2 j-1}-\left(\sum_{n=1}^{N}\left(B\left(a_{n}\right), D\left(a_{n}\right)\right) \odot x_{k}\right)_{2 j-1}\right\|,\right. \\
& \left.\left\|\left(T\left(\sum_{n=1}^{N}\left(0, a_{n}\right) \odot x_{k}\right)\right)_{2 j}-\left(\sum_{n=1}^{N}\left(B\left(a_{n}\right), D\left(a_{n}\right)\right) \odot x_{k}\right)_{2 j}\right\|\right\}<\frac{\varepsilon}{4} \\
& \text { onde } j \text { é a projeção do vetor no espaço } \mathcal{W}_{j} .
\end{aligned}
$$


Fixemos então, $\varepsilon>0$ (determinado na construção dos vetores $a_{n}$ ) e $N \in \mathbb{N}$, $N>\left(\frac{4\|T\|}{\varepsilon}\right)^{2}$. Podemos por (2.19) determinar $k \in N_{2}, k=\operatorname{máx}\left\{k_{j_{i}}: 1 \leq i \leq N\right\}$, tal que

$$
\left\|\left[T\left(\sum_{n=1}^{N}\left(0, a_{n}\right) \odot x_{k}\right)-\sum_{n=1}^{N}\left(B\left(a_{n}\right), D\left(a_{n}\right)\right) \odot x_{k}\right]_{j_{i}}\right\|_{V}<\frac{\varepsilon}{4},
$$

para cada $i=1,2, \ldots, N$.

Lembremos que $j_{1}, j_{2}, \ldots, j_{N}$ é parte da seqüência crescente $\left(j_{i}\right)_{i=1}^{\infty}$ construída anteriormente e a norma é a de um vetor do espaço $\mathcal{W}_{j_{i}}$.

Podemos agora, para cada $i=1,2, \ldots, N$, considerando $v_{k_{j_{i}}}^{*} \in \mathcal{W}_{j_{i}}^{*}$ e $k \in N_{2}$ escolhido, obter:

$$
\begin{aligned}
& \left|v_{k_{j_{i}}}^{*}\left(\sum_{n=1}^{N}\left(B\left(a_{n}\right), D\left(a_{n}\right)\right) \odot x_{k}\right)\right| \leq\left|v_{k_{j_{i}}}^{*}\left(T\left(\sum_{n=1}^{N}\left(0, a_{n}\right) \odot x_{k}\right)\right)\right| \\
+ & \left|v_{k_{j_{i}}}^{*}\left(T\left(\sum_{n=1}^{N}\left(0, a_{n}\right) \odot x_{k}\right)-\sum_{n=1}^{N}\left(B\left(a_{n}\right), D\left(a_{n}\right)\right) \odot x_{k}\right)\right| \\
\leq & \left|v_{k_{j_{i}}}^{*}\left(T\left(\sum_{n=1}^{N}\left(0, a_{n}\right) \odot x_{k}\right)\right)\right|+\left\|v_{k_{j_{i}}}^{*}\right\|||\left[T\left(\sum_{n=1}^{N}\left(0, a_{n}\right) \odot x_{k}\right)\right. \\
& \left.-\sum_{n=1}^{N}\left(B\left(a_{n}\right), D\left(a_{n}\right)\right) \odot x_{k}\right]\left._{j_{i}}\right|_{V} \leq\left|v_{k_{j_{i}}}^{*}\left(T\left(\sum_{n=1}^{N}\left(0, a_{n}\right) \odot x_{k}\right)\right)\right|+\frac{\varepsilon}{4} .
\end{aligned}
$$

Concluímos então que:

$$
\left|v_{k_{j_{i}}}^{*}\left(T\left(\sum_{n=1}^{N}\left(0, a_{n}\right) \odot x_{k}\right)\right)\right| \geq\left|v_{k_{j_{i}}}^{*}\left(\sum_{n=1}^{N}\left(B\left(a_{n}\right), D\left(a_{n}\right)\right) \odot x_{k}\right)\right|-\frac{\varepsilon}{4}
$$

Olhemos agora para o vetor $\sum_{n=1}^{N}\left(B\left(a_{n}\right), D\left(a_{n}\right)\right) \odot x_{k}$. Pela definição, para cada $n, k \in \mathbb{N}$,

$$
\begin{aligned}
& \left(B\left(a_{n}\right), D\left(a_{n}\right)\right) \odot x_{k}=\ell^{-1}\left(B\left(a_{n}\right), D\left(a_{n}\right)\right) \otimes x_{k} \\
= & \left(\left(B\left(a_{n}\right)\right)_{1},\left(D\left(a_{n}\right)\right)_{1},\left(B\left(a_{n}\right)\right)_{2},\left(D\left(a_{n}\right)\right)_{2}, \ldots,\left(B\left(a_{n}\right)\right)_{j_{1}},\left(D\left(a_{n}\right)\right)_{j_{1}}, \ldots,\right. \\
& \left.\left(B\left(a_{n}\right)\right)_{j_{2}},\left(D\left(a_{n}\right)\right)_{j_{2}}, \ldots,\left(B\left(a_{n}\right)\right)_{j_{N}},\left(D\left(a_{n}\right)\right)_{j_{N}}, \ldots\right) \otimes x_{k} \\
= & \left(\left(B\left(a_{n}\right)\right)_{1} x_{k 1},\left(D\left(a_{n}\right)\right)_{1} x_{k 2}, \ldots,\left(B\left(a_{n}\right)\right)_{j_{1}} x_{k 2 j_{1}-1},\left(D\left(a_{n}\right)\right)_{j_{1}} x_{k 2 j_{1}}, \ldots,\right.
\end{aligned}
$$




$$
\begin{aligned}
& \left.\qquad\left(B\left(a_{n}\right)\right)_{j_{2}} x_{k 2 j_{2}-1},\left(D\left(a_{n}\right)\right)_{j_{2}} x_{k 2 j_{2}}, \ldots,\left(B\left(a_{n}\right)\right)_{j_{N}} x_{k 2 j_{N}-1},\left(D\left(a_{n}\right)\right)_{j_{N}} x_{k 2 j_{N}}, \ldots\right) . \\
& \text { Logo, } \sum_{n=1}^{N}\left(B\left(a_{n}\right), D\left(a_{n}\right)\right) \odot x_{k}=\left(\sum_{n=1}^{N}\left(B\left(a_{n}\right)\right)_{2 j-1} x_{k 2 j-1}, \sum_{n=1}^{N}\left(D\left(a_{n}\right)\right)_{2 j} x_{k 2 j}\right)_{j=1}^{\infty}, \\
& \text { onde }\left(\sum_{n=1}^{N}\left(B\left(a_{n}\right)\right)_{2 j-1} x_{k 2 j-1}, \sum_{n=1}^{N}\left(D\left(a_{n}\right)\right)_{2 j} x_{k 2 j}\right) \text { é um vetor do espaço } \mathcal{W}_{j} .
\end{aligned}
$$

Voltando ao resultado (2.20), tínhamos para cada $i=1,2, \ldots, N$,

$$
\begin{aligned}
& \left|v_{k_{j_{i}}}^{*}\left(T\left(\sum_{n=1}^{N}\left(0, a_{n}\right) \odot x_{k}\right)\right)\right| \geq\left|v_{k_{j_{i}}}^{*}\left(\sum_{n=1}^{N}\left(B\left(a_{n}\right), D\left(a_{n}\right)\right) \odot x_{k}\right)\right|-\frac{\varepsilon}{4} \\
= & \left|\left(x_{k}^{*}, 0\right)\left(\sum_{n=1}^{N} B\left(a_{n}\right)_{j_{i}} x_{k 2 j_{i}-1}, \sum_{n=1}^{N}\left(D\left(a_{n}\right)\right)_{j_{i}} x_{k 2 j_{i}}\right)\right|-\frac{\varepsilon}{4} \\
= & \left|x_{k}^{*}\left(\sum_{n=1}^{N}\left(B\left(a_{n}\right)\right)_{j_{i}} x_{k 2 j_{i}-1}\right)\right|-\frac{\varepsilon}{4}=\left|\sum_{n=1}^{N}\left(B\left(a_{n}\right)\right)_{j_{i}}\right|-\frac{\varepsilon}{4} \\
\geq & \left|B\left(a_{i}\right)_{j_{i}}\right|-\sum_{\substack{n=1 \\
n \neq i}}^{N}\left|B\left(a_{n}\right)_{j_{i}}\right|-\frac{\varepsilon}{4} \geq \varepsilon-\frac{\varepsilon}{2}-\frac{\varepsilon}{4}=\frac{\varepsilon}{4} .
\end{aligned}
$$

Agora, o vetor $T\left(\sum_{n=1}^{N}\left(0, a_{n}\right) \odot x_{k}\right) \in \mathbf{W}$. Logo, $T\left(\sum_{n=1}^{N}\left(0, a_{n}\right) \odot x_{k}\right)=\sum_{j=1}^{\infty} \mathrm{w}_{j}$.

Assim, pela definição de norma, pela Observação 1.19 e sabendo que $k \in N_{2}$, ob-

$$
\begin{aligned}
& \text { teremos }\left\|T\left(\sum_{n=1}^{N}\left(0, a_{n}\right) \odot x_{k}\right)\right\|^{2} \geq\left\|\sum_{j=1}^{j_{N}} \mathrm{w}_{j}\right\|_{V}^{2} \geq \sum_{j=1}^{j_{N}}\left|v_{k j}^{*}\left(T\left(\sum_{n=1}^{N}\left(0, a_{n}\right) \odot x_{k}\right)\right)\right|^{2} \geq \\
& \sum_{i=1}^{N}\left|v_{k j_{i}}^{*}\left(T\left(\sum_{n=1}^{N}\left(0, a_{n}\right) \odot x_{k}\right)\right)\right|^{2} \geq N \frac{\varepsilon^{2}}{16}>\left(\frac{4\|T\|}{\varepsilon}\right)^{2} \frac{\varepsilon^{2}}{16}=\|T\|^{2} .
\end{aligned}
$$

Concluímos que, se $B$ não é estritamente singular, teremos $\left\|T\left(\sum_{n=1}^{N}\left(0, a_{N}\right) \odot x_{k}\right)\right\|$ $>\|T\|$, o que é uma contradição, já que $\left\|\sum_{n=1}^{N}\left(0, a_{n}\right) \odot x_{k}\right\|_{V}=1$ e $T: \mathbf{W} \rightarrow \mathbf{W}$ era pela hipótese um operador limitado.

Raciocínio análogo nos leva a uma contradição, supondo que o operador $C$ não seja estritamente singular. 
Neste caso, encontramos uma seqüência $\left(a_{n}\right)_{n=1}^{\infty}$ de vetores de $c_{0}$, normalizada, com $a_{n}<a_{n+1}, \forall n \in \mathbb{N}$, e uma seqüência crescente $\left(j_{i}\right)_{i=1}^{\infty}$ de naturais, tais que $\left|C\left(a_{n}\right)_{j_{i}}\right|>\varepsilon$ se $n=i$ e $\sum_{n \neq i}\left|\left(C\left(a_{n}\right)\right)_{j_{i}}\right|<\frac{\varepsilon}{2}$.

Pelo Lema 2.10, temos que $T\left(\left(a_{n}, 0\right) \odot x_{k}\right)-\tilde{T}_{\Lambda}\left(a_{n}, 0\right) \odot x_{k}$ converge pontualmente a zero se $k \rightarrow+\infty$, para todo $n \in \mathbb{N}$, e pela definição dos operadores $A$ e $C$, $\tilde{T}_{\Lambda}\left(a_{n}, 0\right)=\left(A\left(a_{n}\right), C\left(a_{n}\right)\right)$.

Para todo $i, j, k$ e $n \in \mathbb{N}$, temos $w_{i j}^{*}\left(\left(a_{n}, 0\right) \odot x_{k}\right)=0$, pois a segunda componente da projeção $P_{j}$ do vetor $\left(a_{n}, 0\right) \odot x_{k}$ em $\mathcal{W}_{j}$ é igual a zero, isto é, $P_{j}\left(\left(a_{n}, 0\right) \odot x_{k}\right)=$ $a_{n j} x_{k 2 j}+0 \in \mathcal{W}_{j}$. Se $k \in N_{3}$ e $i \in N_{1} \cup N_{2} \cup N_{4}$, o mesmo ocorre com $u_{i j}^{*}$ e $v_{i j}^{*}$, pois $i \neq k$.

Assim, pela definição de norma, se $k \in N_{3}$ e $N \in \mathbb{N}$, obtemos como no caso anterior que $\left\|\sum_{n=1}^{N}\left(a_{n}, 0\right) \odot x_{k}\right\|_{V}=\left\|\sum_{n=1}^{N} a_{n}\right\|_{\infty}=1$. Estimamos a norma do vetor $T\left(\sum_{n=1}^{N}\left(a_{n}, 0\right) \odot x_{k}\right)$ da mesma forma que fizemos no caso do operador $B$ e encontramos $k \in N_{3}$ e $N \in \mathbb{N}$, tais que $\left\|T\left(\sum_{n=1}^{N}\left(a_{n}, 0\right) \odot x_{k}\right)\right\| \geq\|T\|$, o que é uma contradição, pois $T$ é um operador limitado.

Sabendo que $B$ e $C$ são operadores de $c_{0}$ estritamente singulares, vamos supor para finalizar, que o opera.dor $A-D$ não seja.

Neste caso, obtemos utilizando argumentos semelhantes aos casos anteriores, $\varepsilon>0$, uma seqüência $\left(a_{n}\right)_{n=1}^{\infty}$ de vetores de $c_{0}$ normalizada com $a_{n}<a_{n+1}, \forall n \in \mathbb{N}$ e uma seqüência crescente $\left(j_{i}\right)_{i=1}^{\infty}$ de naturais, tais que $\left|\left((A-D)\left(a_{n}\right)\right)_{j_{i}}\right|>\varepsilon$ se $n=i$ $\mathrm{e} \sum_{n \neq i}\left|\left((A-D)\left(a_{n}\right)\right)_{j_{i}}\right|<\frac{\varepsilon}{4}$

Além disso, é possível determinar uma subseqüência de $\left(a_{n}\right)_{n=1}^{\infty}$ que denotaremos 
novamente por $\left(a_{n}\right)_{n=1}^{\infty}$ com a propriedade adicional: máx $\left(\left\|B\left(a_{n}\right)\right\|_{\infty},\left\|C\left(a_{n}\right)\right\|_{\infty}\right)<$ $\varepsilon 10^{-n}$, para todo $n \in \mathbb{N}$. Mostremos para isso que $\lim _{n \rightarrow+\infty} B\left(a_{n}\right)=\lim _{n \rightarrow+\infty} C\left(a_{n}\right)=0$.

Suponhamos que não ocorra para $B, \log \|\| B\left(a_{n}\right) \| \not \rightarrow 0$ e como $B\left(a_{n}\right) \stackrel{w}{\longrightarrow} 0$, pela. Proposição 0.10 , existe uma subseqüência $\left(B\left(a_{n_{k}}\right)\right)_{k=1}^{\infty}$ de $\left(B\left(a_{n}\right)\right)_{n=1}^{\infty}$, que é uma seqüência básica, equivalente a uma base de bloco da base $\left(e_{i}\right)_{i=1}^{\infty}$ de $c_{0}$.

Temos também $a_{n_{k}} \stackrel{w}{\longrightarrow} 0 \mathrm{e}\left\|a_{n_{k}}\right\|=1$, para todo $k \in \mathbb{N}$, utilizando novamente a Proposição 0.10 existe uma subseqüência de $\left(a_{n_{k}}\right)_{k=1}^{\infty},\left(a_{n_{k_{i}}}\right)_{i=1}^{\infty}$ que é uma seqüência básica e $\left(a_{n_{k_{i}}}\right)_{i=1}^{\infty} \sim\left(u_{i}\right)_{i=1}^{\infty}$, onde $\left(u_{i}\right)_{i=1}^{\infty}$ é base de bloco de $\left(e_{i}\right)_{i=1}^{\infty}$. Observemos que a subseqüência $\left(B\left(a_{n_{k_{i}}}\right)\right)_{i=1}^{\infty}$ de $\left(B\left(a_{n_{k}}\right)\right)_{k=1}^{\infty}$ é também uma seqüência básica e $\left(B\left(a_{n_{k_{i}}}\right)\right)_{i=1}^{\infty} \sim\left(v_{i}\right)_{i=1}^{\infty}$, onde $\left(v_{i}\right)_{i=1}^{\infty}$ é base de bloco de $\left(e_{i}\right)_{i=1}^{\infty}$.

As seqüências normalizadas, $\left(\frac{u_{i}}{\left\|u_{i}\right\|}\right)_{i=1}^{\infty}$ e $\left(\frac{v_{i}}{\left\|v_{i}\right\|}\right)_{i=1}^{\infty}$, são bases de bloco de $\left(e_{i}\right)_{i=1}^{\infty}$. Logo, pela Proposição 0.9, $\left(\frac{u_{i}}{\left\|u_{i}\right\|}\right)_{i=1}^{\infty} \sim\left(e_{i}\right)_{i=1}^{\infty}$ e $\left(\frac{v_{i}}{\left\|v_{i}\right\|}\right)_{i=1}^{\infty} \sim\left(e_{i}\right)_{i=1}^{\infty}$; mas $\left(a_{n_{k_{i}}}\right)_{i=1}^{\infty} \sim$ $\left(\frac{u_{i}}{\left\|u_{i}\right\|}\right)_{i=1}^{\infty}$ e $\left(B\left(a_{n_{k_{i}}}\right)\right)_{i=1}^{\infty} \sim\left(\frac{v_{i}}{\left\|v_{i}\right\|}\right)_{i=1}^{\infty}$, o que acarreta $\left(a_{n_{k_{i}}}\right)_{i=1}^{\infty} \sim\left(B\left(a_{n_{k_{i}}}\right)\right)_{i=1}^{\infty}$.

Considerando $A=\left[\left(a_{n_{k_{i}}}\right)_{i=1}^{\infty}\right]$ e $Y=\left[\left(B\left(a_{n_{k_{i}}}\right)\right)_{i=1}^{\infty}\right]$, temos pela Proposição 0.8 que $B: A \rightarrow Y$ é isomorfismo, o que contraria a hipótese que fizemos de que $B$ é estritamente singular. Raciocínio análogo pode ser aplicado ao operador $C$.

Como nos casos anteriores, pelo Lema 2.10, obtemos que $T\left(\left(a_{n}, a_{n}\right) \odot x_{k}\right)-$ $\tilde{T}_{\Lambda}\left(a_{n}, a_{n}\right) \odot x_{k}$ converge pontualmente a zero se $k \rightarrow+\infty$, onde, pela definição dos operadores $A, B, C$ e $D, \tilde{T}_{\Lambda}\left(a_{n}, a_{n}\right)=\left((A+B)\left(a_{n}\right),(C+D)\left(a_{n}\right)\right)$.

Notemos também que para todo $i, j, k$ e $n \in \mathbb{N}$, temos $u_{i j}^{*}\left(\left(a_{n}, a_{n}\right) \odot x_{k}\right)=0$, pois $\left(x_{i}^{*}-x_{i}^{*}\right)\left(a_{n j} x_{k 2 j-1}, a_{n j} x_{k 2 j}\right)=a_{n j} x_{i}^{*}\left(x_{k 2 j-1}\right)-a_{n j} x_{i}^{*}\left(x_{k 2 j}\right)=0$. Se $k \in N_{1} \mathrm{e}$ $i \in N_{2} \cup N_{3} \cup N_{4}$, o mesmo ocorre com $v_{i j}^{*}$ e $w_{i j}^{*}$, pois $i \neq k$. 
Dado $N \in \mathbb{N}$, consideremos o vetor de $\mathrm{V}, \sum_{n=1}^{\infty}\left(a_{n}, a_{n}\right) \odot x_{k}$. Se $k \in N_{1}$ e $N \in \mathbb{N}$, obtemos como nos casos anteriores que $\left\|\sum_{n=1}^{N}\left(a_{n}, a_{n}\right) \odot x_{k}\right\|_{V}=\left\|\sum_{n=1}^{N} a_{n}\right\|_{\infty}=1$.

Concluímos também que para todo $n \in \mathbb{N}, T\left(\sum_{n=1}^{N}\left(a_{n}, a_{n}\right) \odot x_{k}\right)-\sum_{n=1}^{N}((A+$ $\left.B)\left(a_{n}\right),(C+D)\left(a_{n}\right)\right) \odot x_{k}$ converge pontualmente a zero, se $k \rightarrow+\infty$.

Segue que, dados $N \in \mathbb{N}, \varepsilon>0$ e $j \in \mathbb{N}$, podemos determinar $k_{j} \in \mathbb{N}$, tal que se $k>k_{j}$,

$$
\left\|\left[T\left(\sum_{n=1}^{N}\left(a_{n}, a_{n}\right) \odot x_{k}\right)-\sum_{n=1}^{N}\left((A+B)\left(a_{n}\right),(C+D)\left(a_{n}\right)\right) \odot x_{k}\right]_{j}\right\|_{V} \leq \frac{\varepsilon}{4} .
$$

Fixemos $\varepsilon>0$ (determinado na construção dos vetores $a_{n}$ ) e $N \in \mathbb{N}, N>$ $\left(\frac{4\|T\|^{2}}{\varepsilon}\right)$. Podemos por (2.21) determinar $k \in N_{1}, k=\operatorname{máx}\left\{k_{j_{i}}: 1 \leq i \leq N\right\}$ tal que

$$
\left\|\left[T\left(\sum_{n=1}^{N}\left(a_{n}, a_{n}\right) \odot x_{k}\right)-\sum_{n=1}^{N}\left((A+B)\left(a_{n}\right),(C+D)\left(a_{n}\right)\right) \odot x_{k}\right]_{j_{i}}\right\|_{V} \leq \frac{\varepsilon}{4},
$$

para cada $i=1,2, \ldots, N$.

Considerando $u_{k j_{i}}^{*} \in \mathcal{W}_{j_{i}}^{*}$ e $k \in N_{1}$ escolhido, podemos obter como nos casos anteriores:

$$
\left|u_{k j_{i}}^{*}\left(T\left(\sum_{n=1}^{N}\left(a_{n}, a_{n}\right) \odot x_{k}\right)\right)\right| \geq\left|u_{k j_{i}}^{*}\left(\sum_{n=1}^{N}\left((A+B)\left(a_{n}\right),(C+D)\left(a_{n}\right)\right) \odot x_{k}\right)\right|-\frac{\varepsilon}{4} .
$$

Agora, neste caso, o vetor $\left((A+B)\left(a_{n}\right),(C+D)\left(a_{n}\right)\right) \odot x_{k}$ é igual a.

$$
\begin{aligned}
& \ell^{-1}\left((A+B)\left(a_{n}\right),(C+D)\left(a_{n}\right)\right) \otimes x_{k} \\
= & \left(\left[\left(A\left(a_{n}\right)\right)_{1}+\left(B\left(a_{n}\right)\right)_{1}\right],\left[\left(C\left(a_{n}\right)\right)_{1}+\left(D\left(a_{n}\right)\right)_{1}\right],\left[\left(A\left(a_{n}\right)\right)_{2}+\left(B\left(a_{n}\right)\right)_{2}\right],\right. \\
& {\left[\left(C\left(a_{n}\right)\right)_{2}+\left(D\left(a_{n}\right)\right)_{2}\right], \ldots,\left[\left(A\left(a_{n}\right)\right)_{j_{1}}+\left(B\left(a_{n}\right)\right)_{j_{1}}\right],\left[\left(C\left(a_{n}\right)\right)_{j_{1}}+\left(D\left(a_{n}\right)\right)_{j_{1}}\right], \ldots, } \\
& {\left.\left[\left(A\left(a_{n}\right)\right)_{j_{N}}+\left(B\left(a_{n}\right)\right)_{j_{N}}\right],\left[\left(C\left(a_{n}\right)\right)_{j_{N}}+\left(D\left(a_{n}\right)\right)_{j_{N}}\right], \ldots\right) \otimes x_{k} }
\end{aligned}
$$




$$
\begin{aligned}
= & \left(\left[\left(A\left(a_{n}\right)\right)_{1}+\left(B\left(a_{n}\right)\right)_{1}\right] x_{k 1},\left[\left(C\left(a_{n}\right)\right)_{1}+\left(D\left(a_{n}\right)\right)_{1}\right] x_{k 2}, \ldots,\right. \\
& {\left[\left(A\left(a_{n}\right)\right)_{j_{1}}+\left(B\left(a_{n}\right)\right)_{j_{1}}\right] x_{k 2 j_{1}-1},\left[\left(C\left(a_{n}\right)\right)_{j_{1}}+\left(D\left(a_{n}\right)\right)_{j_{1}}\right] x_{k 2 j_{1}}, \ldots, } \\
& {\left.\left[\left(A\left(a_{n}\right)\right)_{j_{N}}+\left(B\left(a_{n}\right)\right)_{j_{N}}\right] x_{k 2 j_{N}-1},\left[\left(C\left(a_{n}\right)\right)_{j_{N}}+\left(D\left(a_{n}\right)\right)_{j_{N}}\right] x_{k 2 j_{N}}, \ldots\right) . }
\end{aligned}
$$

Assim,

$$
\begin{aligned}
& \sum_{n=1}^{N}\left((A+B)\left(a_{n}\right),(C+D)\left(a_{n}\right)\right) \odot x_{k} \\
= & \left(\sum_{n=1}^{N}\left[\left(A\left(a_{n}\right)\right)_{2 j-1}+\left(B\left(a_{n}\right)\right)_{2 j-1}\right] x_{k 2 j-1}, \sum_{n=1}^{N}\left[\left(C\left(a_{n}\right)\right)_{2 j}+\left(B\left(a_{n}\right)\right)_{2 j}\right] x_{k 2 j}\right)_{j=1}^{\infty},
\end{aligned}
$$

onde $\left(\sum_{n=1}^{N}\left[\left(A\left(a_{n}\right)\right)_{2 j-1}+\left(B\left(a_{n}\right)\right)_{2 j-1}\right] x_{k 2 j-1}, \sum_{n=1}^{N}\left[\left(C\left(a_{n}\right)\right)_{2 j}+\left(B\left(a_{n}\right)\right)_{2 j}\right] x_{k 2 j}\right)$ é um vetor do espaço $\mathcal{W}_{j}$.

Voltando ao resultado (2.22), tínhamos para cada $i=1,2, \ldots, N$,

$$
\begin{aligned}
& \left|u_{k j_{i}}^{*}\left(T\left(\sum_{n=1}^{N}\left(a_{n}, a_{n}\right) \odot x_{k}\right)\right)\right| \geq\left|u_{k j_{i}}^{*}\left(\sum_{n=1}^{N}\left((A+B)\left(a_{n}\right),(C+D)\left(a_{n}\right)\right) \odot x_{k}\right)\right|-\frac{\varepsilon}{4} \\
& =\left|\left(x_{k}^{*},-x_{k}^{*}\right)\left(\sum_{n=1}^{N}\left[\left(A\left(a_{n}\right)\right)_{j_{i}}+\left(B\left(a_{n}\right)\right)_{j_{i}}\right] x_{k 2 j_{i}-1}, \sum_{n=1}^{N}\left[\left(C\left(a_{n}\right)\right)_{j_{i}}+\left(D\left(a_{n}\right)\right)_{j_{i}}\right] x_{k 2 j_{i}}\right)\right|-\frac{\varepsilon}{4} \\
& =\left|\sum_{n=1}^{N}\left[\left(A\left(a_{n}\right)\right)_{j_{i}}+\left(B\left(a_{n}\right)\right)_{j_{i}}\right]+\sum_{n=1}^{N}\left[\left(-C\left(a_{n}\right)\right)_{j_{i}}+\left(-D\left(a_{n}\right)\right)_{j_{i}}\right]\right|-\frac{\varepsilon}{4} \\
& =\left|\sum_{n=1}^{N}\left[\left(A\left(a_{n}\right)\right)_{j_{i}}-\left(D\left(a_{n}\right)\right)_{j_{i}}\right]+\sum_{n=1}^{N}\left[\left(B\left(a_{n}\right)\right)_{j_{i}}-\left(C\left(a_{n}\right)\right)_{j_{i}}\right]\right|-\frac{\varepsilon}{4} \\
& \geq\left|\sum_{n=1}^{N}\left[\left(A\left(a_{n}\right)\right)_{j_{i}}-\left(D\left(a_{n}\right)\right)_{j_{i}}\right]\right|-\left|\sum_{n=1}^{N}\left[\left(B\left(a_{n}\right)\right)_{j_{i}}-\left(C\left(a_{n}\right)\right)_{j_{i}}\right]\right|-\frac{\varepsilon}{4} \\
& \geq\left|\sum_{n=1}^{N}\left[\left(A\left(a_{n}\right)\right)_{j_{i}}-\left(D\left(a_{n}\right)\right)_{j_{i}}\right]\right|-\sum_{n=1}^{N}\left|\left[\left(B\left(a_{n}\right)\right)_{j_{i}}-\left(C\left(a_{n}\right)\right)_{j_{i}}\right]\right|-\frac{\varepsilon}{4} \\
& \geq\left|\sum_{n=1}^{N}\left[\left(A\left(a_{n}\right)\right)_{j_{i}}-\left(D\left(a_{n}\right)\right)_{j_{i}}\right]\right|-\sum_{n=1}^{N}\left|\left(B\left(a_{n}\right)\right)_{j_{i}}\right|-\sum_{n=1}^{N}\left|\left(C\left(a_{n}\right)\right)_{j_{i}}\right|-\frac{\varepsilon}{4} \\
& \geq \mid\left((A-D)\left(a_{i}\right)_{j_{i}}\left|-\sum_{\substack{n=1 \\
n \neq i}}^{N}\right|\left((A-D)\left(a_{n}\right)\right)_{j_{i}} \mid-\sum_{n=1}^{\infty} \frac{\varepsilon}{10^{n}}-\sum_{n=1}^{\infty} \frac{\varepsilon}{10^{n}}-\frac{\varepsilon}{4}\right. \\
& \geq \varepsilon-\frac{\varepsilon}{4}-\frac{2 \varepsilon}{9}-\frac{\varepsilon}{4}>\frac{\varepsilon}{4} .
\end{aligned}
$$


Assim, obtemos como nos casos anteriores $\left\|T\left(\sum_{n=1}^{N}\left(a_{n}, a_{n}\right) \odot x_{k}\right)\right\| \geq\|T\|$, o que é uma contradição.

Finalmente estamos em condições de demonstrar o principal resultado dessa. dissertação.

Teorema 2.22 O espaço $X \oplus \mathrm{W}$ é isomorfo ao seu cubo, mas não ao seu quadrado.

Demonstração: Mostraremos que o espaço W não é isomorfo ao seu subespaço $X \oplus \sum_{j=2}^{\infty} \oplus \mathcal{W}_{j}$, onde podemos pensar em $X$ como sendo a segunda componente $\left(X_{2}\right)$ do espaço $\mathcal{W}_{1}$.

Como já vimos no Lema 1.22, item (a), $X \oplus \sum_{j=2}^{\infty} \mathcal{W}_{j} \sim X \oplus \mathrm{W}$, logo se $\mathrm{W} \nsim$ $X \oplus \sum_{j=2}^{\infty} \oplus \mathcal{W}_{j}$, então $\mathbf{W} \nsim X \oplus \mathbf{W}$ e, conseqüentemente, pelo Lema 1.22 , item (b), $(X \oplus \mathbf{W})^{2} \sim \mathrm{W} \not X \oplus \mathbf{W}$.

Suponhamos então que exista um isomorfismo $T: W \rightarrow X \oplus \sum_{j=2}^{\infty} \oplus \mathcal{W}_{j}$. Seja $P: \mathrm{W} \rightarrow \mathrm{W}$ a projeção de $\mathrm{W}$ no seu subespaço $X \oplus \sum_{j=2}^{\infty} \oplus \mathcal{W}_{j}$.

Consideremos também $S: \mathrm{W} \rightarrow \mathrm{W}$ a inversa à esquerda de $T$, definida por $\left(T^{-1} P\right)(a), \forall a \in \mathrm{W}$.

Assim, $S T: \mathbf{W} \rightarrow \mathbf{W}$ é tal que $S T=T^{-1} P T=I d_{\mathbf{W}}$ e $T S: \mathbf{W} \rightarrow X \oplus \sum_{j=2}^{\infty} \mathcal{W}_{j}$ é tal que $T S=T T^{-1} P=P$.

Verifiquemos inicialmente que $\Phi\left(I d_{\mathrm{W}}\right)=I d_{c_{0}}$ e que $\Phi(P)=Q$, onde $Q: c_{0} \rightarrow c_{0}$ é a projeção de $c_{0}$ no seu subespaço fechado $\left[\left(e_{j}\right)_{j=2}^{\infty}\right]$, isto é, dado $c \in c_{0}, c=\sum_{j=1}^{\infty} \alpha_{j} e_{j}$, temos $Q(c)=\sum_{j=2}^{\infty} \alpha_{j} e_{j}$.

Antes, porém, lembremos que $\Phi\left(I d_{\mathrm{W}}\right)=I_{\Lambda}: c_{0} \rightarrow c_{0}$ e $I_{\Lambda}(a)=\Lambda_{I d_{\mathrm{W}}} a, \forall a \in c_{00}$, 
onde $\Lambda_{I d_{\mathbf{W}}}$ é a matriz de termo geral $\alpha_{i j}$, com a propriedade de que $\lim _{n \rightarrow+\infty} I_{i j}\left(x_{n j}\right)$ $\alpha_{i j}\left(x_{n i}\right)=0, \forall i, j \in \mathbb{N}$ e $I_{i j}: X_{j} \rightarrow X_{i}$ é o operador definido por $I_{i j}=\left.P_{i} I d_{\mathrm{W}}\right|_{X_{j}}$.

Assim, se $i \neq j$, então $I_{i j}\left(x_{n j}\right)=\left(\left.P_{i} I d_{\mathrm{W}}\right|_{X_{j}}\right)\left(x_{n j}\right)=\left(P_{i} I d_{\mathrm{W}}\right)\left(x_{n j}\right)=P_{i}\left(x_{n j}\right)=$ 0 , para todo $n \in \mathbb{N}$. Logo, de $\lim _{n \rightarrow+\infty} I_{i j}\left(x_{n j}\right)-\alpha_{i j}\left(x_{n i}\right)=0$ temos $\lim _{n \rightarrow+\infty}-\alpha_{i j}\left(x_{n i}\right)=0$ e como $\left\|x_{n i}\right\|=1, \forall n \in \mathbb{N}$, temos $\alpha_{i j}=0$.

Se $i=j$, então $I_{i j}\left(x_{n j}\right)=\left(\left.P_{i} d_{\mathbf{W}}\right|_{X_{j}}\right)\left(x_{n j}\right)=\left(P_{i} I d_{\mathrm{W}}\right)\left(x_{n j}\right)=P_{i}\left(x_{n j}\right)=x_{n j}$, para todo $n \in \mathbb{N}$. Assim, de $\lim _{n \rightarrow+\infty} I_{i j}\left(x_{n j}\right)-\alpha_{i j}\left(x_{n i}\right)=0$ temos que $\lim _{n \rightarrow+\infty} x_{n j}-\alpha_{i j} x_{n i}=0$, $\operatorname{logo} \lim _{n \rightarrow+\infty} x_{n j}-\alpha_{i j} x_{n j}=0$, portanto $\lim _{n \rightarrow+\infty}\left(1-\alpha_{i j}\right) x_{n j}=0$ e como $\left\|x_{n j}\right\|=1$, $\forall n \in \mathbb{N}$, temos $1-\alpha_{i j}=0$, isto é, $\alpha_{i j}=1$.

Concluímos que $\Lambda_{I d_{\mathbf{W}}}$ é a matriz identidade e que $\Phi\left(I d_{\mathrm{W}}\right)=I_{\Lambda}$ é o operador $I d_{c_{0}}$.

Da mesma forma, temos $\Phi(P)=P_{\Lambda}: c_{0} \rightarrow c_{0}$ e $P_{\Lambda}(a)=\Lambda_{P}(a), \forall a \in c_{00}$, onde $\Lambda_{P}$ é a matriz de termo geral $\beta_{i j}$, tal que $\lim _{n \rightarrow+\infty} P_{i j}\left(x_{n j}\right)-\beta_{i j}\left(x_{n i}\right)=0, \forall i, j \in \mathbb{N}$ e $P_{i j}: X_{j} \rightarrow X_{i}$ é o operador definido por $P_{i j}=\left.P_{i} P\right|_{X_{j}}$.

Neste caso, se $i=1$ e $j \in \mathbb{N}, j \neq 1$, temos $P_{1 j}\left(x_{n j}\right)=\left(\left.P_{1} P\right|_{X_{j}}\right)\left(x_{n j}\right)=$ $\left(P_{1} P\right)\left(x_{n j}\right)=P_{1}\left(x_{n j}\right)=0$, para todo $n \in \mathbb{N}$, segue que $\lim _{n \rightarrow+\infty} P_{i j}\left(x_{n j}\right)-\beta_{i j}\left(x_{n i}\right)=0$ logo, $\lim _{n \rightarrow+\infty}-\beta_{i j}\left(x_{n i}\right)=0$ e portanto $\beta_{i j}=0$. No caso em que $i=j=1$, temos $P_{1 j}\left(x_{n 1}\right)=\left(\left.P_{1} P\right|_{X_{1}}\right)\left(x_{n 1}\right)=\left(P_{1} P\right)\left(x_{n 1}\right)=P_{1}\left(P\left(x_{n 1}\right)\right)=P_{1}(0)=0$, para todo $n \in \mathbb{N}, \log 0 \beta_{i j}=0$.

Assim, a matriz $\Lambda_{P}$ tem $\beta_{i j}=0$ se $i=1$ e $j \in \mathbb{N}$, logo $\Lambda_{P}(b)=0$, se $b \in c_{00}$ e $b \in\left[e_{1}\right]$, portanto $\Phi(P)=P_{\Lambda}$ é o operador $Q$ definido anteriormente. Agora, $\Phi(S T)=\Phi\left(I d_{\mathbf{W}}\right)=I d_{c_{0}}$, logo pelo Lema 2.17, temos

$$
\Phi(S) \Phi(T)=I d_{c_{0}}
$$


e também pelo mesmo lema,

$$
\Phi(T) \Phi(S)=\Phi(P)=Q
$$

Finalmente, mostremos que $\operatorname{Ker} \Phi(T)=\{0\}$ e a codimensão de $\operatorname{Im} \Phi(T)$ é igual a. 1 .

Pelo resultado (2.23), temos $\Phi(S)(\Phi(T)(c))=c$, para todo $c \in c_{0}$, logo se $c \in$ $\operatorname{Ker} \Phi(T)$ temos $\Phi(T)(c)=0$ e conseqüentemente $\Phi(S)(\Phi(T)(c))=c=\Phi(S)(0)=$ 0.

Temos também que $\Phi(S)$ é sobre $c_{0}$, pois dado $c \in c_{0}$, por $(2.23), c=\Phi(S)$ $(\Phi(T)(c)), \log$ existe $z=\Phi(T)(c) \in c_{0}$ tal que $\Phi(S)(z)=c$. Segue que $\operatorname{dim} \operatorname{Im} \Phi(T)$ $=\operatorname{dim} \operatorname{Im} \Phi(T) \Phi(S)$, mas por (2.24), $\operatorname{dim} \operatorname{Im} \Phi(T) \Phi(S)=\operatorname{dim} \operatorname{Im} Q$, que tem codimensão 1.

Assim, se $T$ fosse isomorfismo, $\Phi(T)=T_{\Lambda}: c_{0} \rightarrow c_{0}$ seria um operador limitado $\operatorname{com} \Phi(T)\left(c_{0}\right)=\left[\left(e_{j}\right)_{j=2}^{\infty}\right]$ e tal que $\operatorname{dim} \operatorname{Ker} \Phi(T)=0$. Mas, $\operatorname{dim} c_{0} /\left[\left(e_{j}\right)_{j=2}^{\infty}\right]=1, \operatorname{logo}$ $\Phi(T)$ seria um operador de Fredholm de índice ímpar, o que contraria o resultado do lema anterior. 


\section{Problemas em aberto}

Terminamos a dissertação apresentando dois problemas que acreditamos ainda estejam em aberto, relacionados com o problema de Schroeder-Bernstein. O primeiro se refere a métodos de decomposição em espaços de Banach.

Problema 1: Sejam $X$ e $Y$ espaços de Banach. Se $X \stackrel{c}{\hookrightarrow} Y, Y \stackrel{c}{\hookrightarrow} X$ e $X^{2} \sim X \oplus Y$, então $X \sim Y$ ?

O problema 1 é importante, pois se tiver uma solução positiva ele unifica os métodos de decomposição de A. Pelczynski apresentados na introdução; pois se $X \sim X^{2}$ e $Y \sim Y^{2}$, então $X^{2} \sim X \oplus Y$ e se $X \sim\left(\sum_{n=1}^{\infty} \oplus X_{n}\right)_{0}$ ou $X \sim\left(\sum_{n=1}^{\infty} \oplus X_{n}\right)_{\ell_{p}}$, então $X^{2} \sim X \oplus Y$, isto é, o problema 1 seria um método mais geral de decomposição para o isomorfismo $X \sim Y$.

Vimos, como um dos principais resultados desta dissertação, que existe um espaço de Banach $Z$, tal que $Z \not Z^{2}$ e $Z \sim Z^{3}$. O segundo problema vai nesta. direção.

Problema 2: Existe um espaço de Banach $X$, tal que $X \nsim X^{2}$ mas $X^{2} \sim X^{3}$ ? 


\section{Referências bibliográficas}

[C] Cristina, C., O primeiro espaço de Banach hereditariamente indecomponível. Instituto de Matemática e Estatística da Universidade de São Paulo, 2000. (Disserta.ção de Mestrado)

[Ca] Casazza, P. G., The Schroeder-Bernstein property for Banach spaces, Contemp. Math., 85, 1989, 61-78.

[Ga] Galego, E. M., Alguns aspectos de geometria de espaços de Banach através de $c_{0}(\mathbb{N})$, $43^{\circ}$ Seminário Brasileiro de Análise, IME-USP, 1996.

[G] Gowers, W. T., A solution to the Schroeder-Bernstein problem for Banach spaces, Bull. London. Math. Soc., 28, 1996, 297-304.

[G-M1] Gowers, W. T.; Maurey, B., The unconditional basic sequence problem, Journal of the American Mathematical Society, 6(4), 1993, 851-874.

[G-M2] Gowers, W. T.; Maurey, B., Banach spaces with small spaces of operators, Math. Ann., 307, 1997, 543-568.

[Ho] Hönig, C. S. Análise Funcional e Aplicações, v.1, IME-USP, 1970.

[L-T] Lindenstrauss, J.; Tzafriri, L., Classical Banach Spaces, v.1, Springer-Verlag, Berlin-Heidelberg-New York, 1977.

[S] Singer, I., Bases in Banach Spaces, v.2, Springer-Verlag, Berlin-Heidelberg-New York, 1981. 